\title{
Paleoseismic Investigations of Stagecoach Road Fault, Southeastern Yucca Mountain, Nye County, Nevada
}

By C.M. Menges, J.A. Oswald, J.A. Coe, S.C. Lundstrom, J.B. Paces, S.A. Mahan, Beth Widmann, and Michele Murray

\section{USGS/OFR--96-417}

U.S. GEOLOGICAL SURVEY

Open-File Report 96-417

Prepared in cooperation with the NEVADA OPERATIONS OFFICE, U.S. DEPARTMENT OF ENERGY, under Interagency Agreement DE-Al08-92NV10874

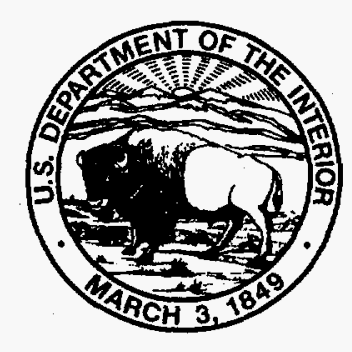




\title{
U.S. DEPARTMENT OF THE INTERIOR BRUCE BABBITT, Secretary
}

\author{
U.S. GEOLOGICAL SURVEY
}

Mark Schaeffer, Acting Director

The use of firm, trade, and brand names in this report is for identification purposes only and does not constitute endorsement by the U.S. Geological Survey.

For additional information write to:

Chief, Earth Science Investigations Program Yucca Mountain Project Branch

U.S. Geological Survey

Box 25046, Mail Stop 421

Denver Federal Center

Denver, CO 80225-0046
Copies of this report can be purchased from:

U.S. Geological Survey

Branch of Information Services

Box 25286

Denver, CO 80225-0286 


\section{DISCLAIMER}

This report was prepared as an account of work sponsored by an agency of the United States Government. Neither the United States Government aor any agency thereof, nor any of their employees, makes any warranty, express or implied, or assumes any legal liability or responsibility for the accuracy, completesess, or usefulness of any information, apparatus, product, or process disclosed, or represents that its use would not infringe privately owned rights. Reference herein to any specific commercial product, process, or service by trade name, trademark, manufacturer, or otherwise does not necessarily constitute or imply its endorsement, recommendation, or favoring by the United States Government or any ageacy thereof. The views and opinions of authors expressed herein do not necessarily state or reflect those of the United States Government or any agency thereof. 


\section{DISCLAIMER}

Portions of this document may be illegible electronic image products. Images are produced from the best available original document. 


\section{CONTENTS}

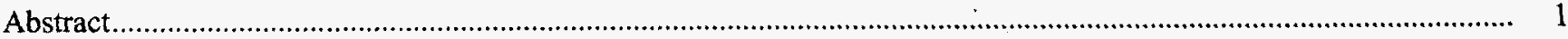

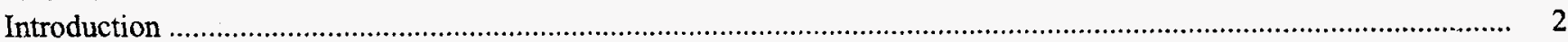

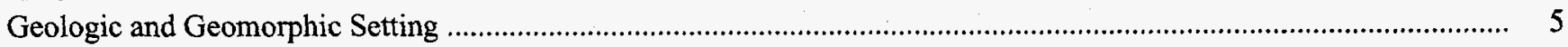

Physiography and Geology of Yucca Mountain Site........................................................................................... 5

Geology and Geomorphology of the Stagecoach Road Fault ......................................................................

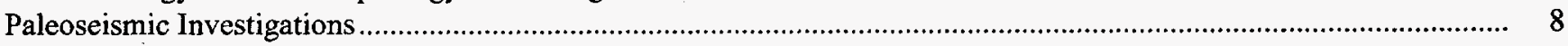

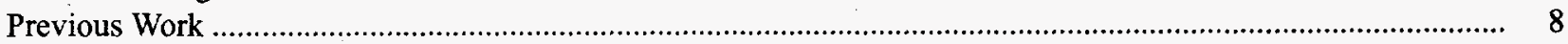

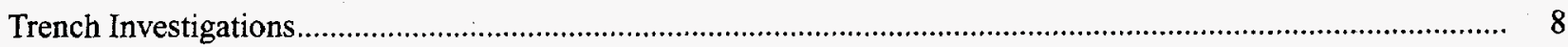

Collection and Interpretation of Paleoseismic Data ................................................................................... 9

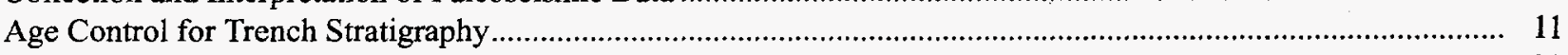

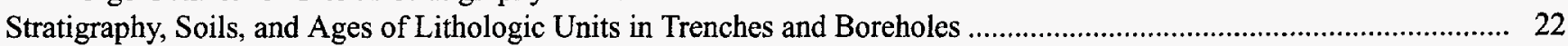

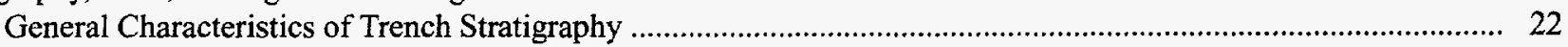

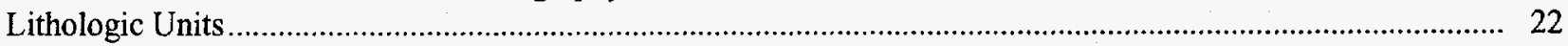

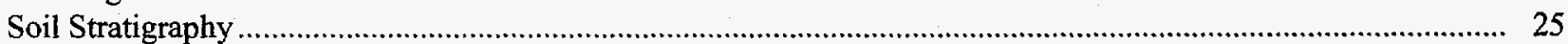

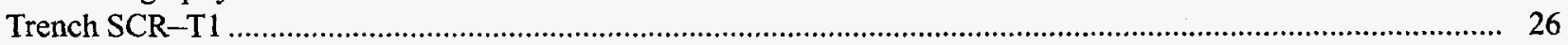

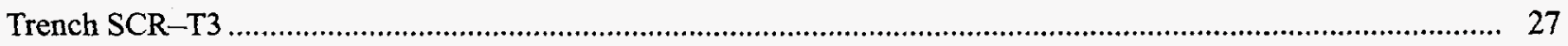

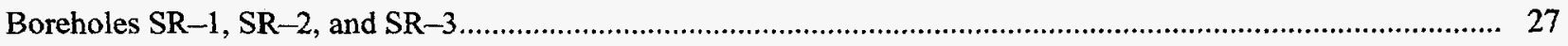

Estimated Ages of Stratigraphic Sequences ................................................................................................ 27

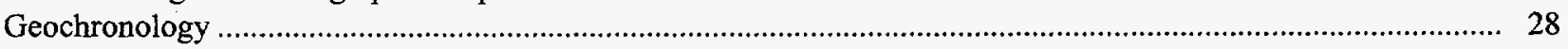

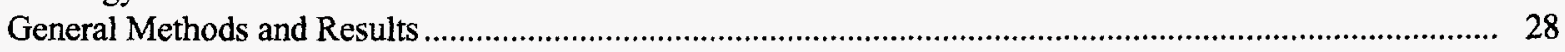

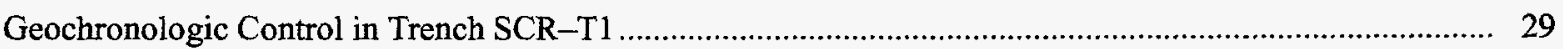

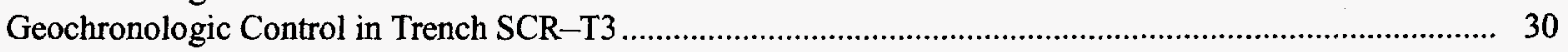

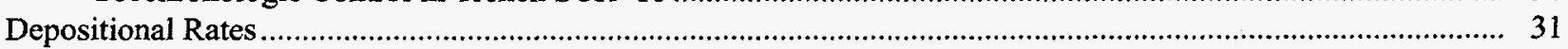

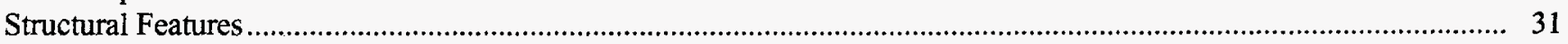

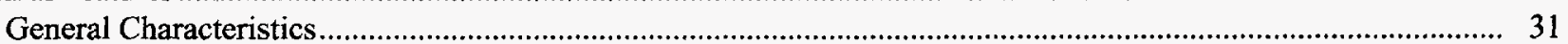

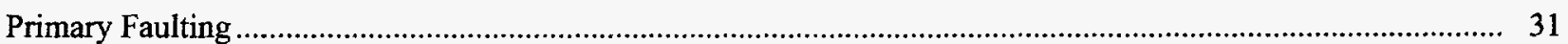

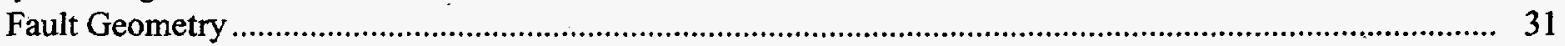

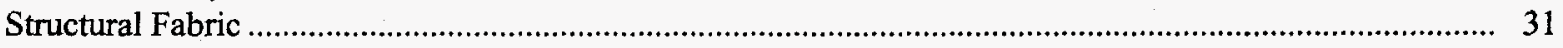

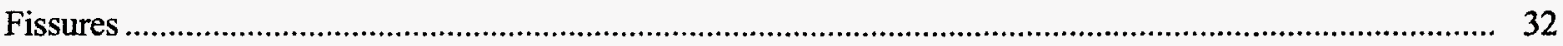

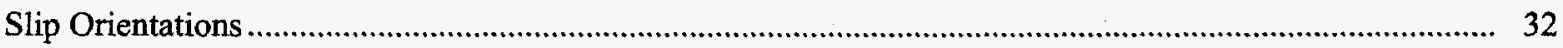

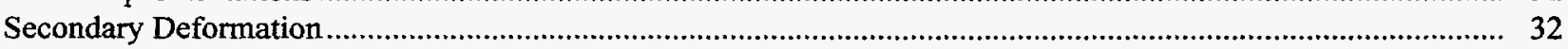

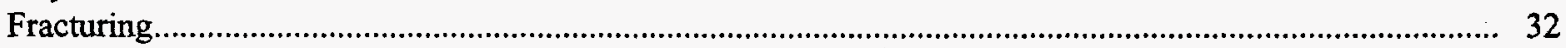

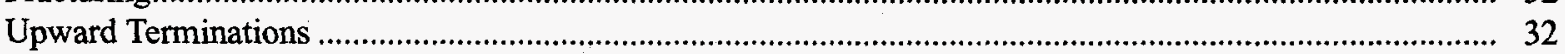

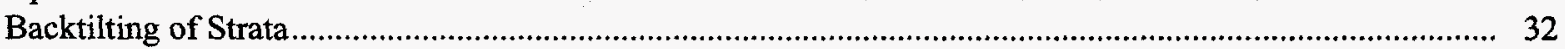

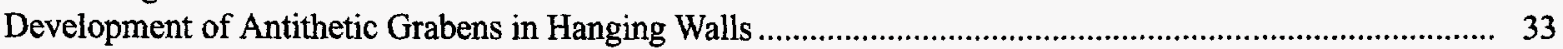

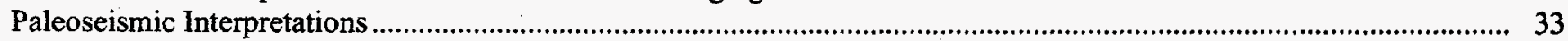

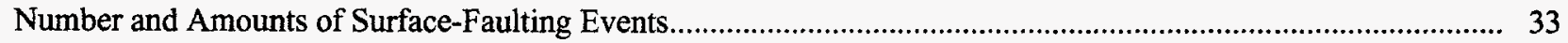

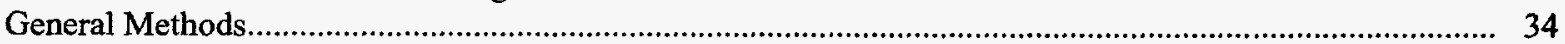

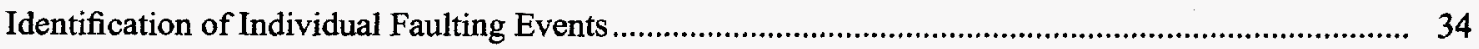

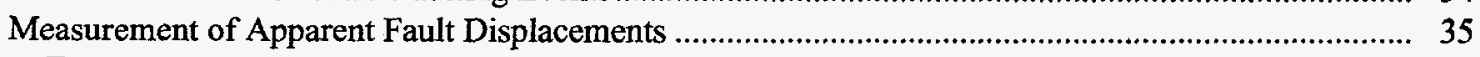

Faulting Events and Apparent Displacements in Trench SCR-T1 .................................................... 36

Faulting Events and Apparent Displacements in Trench SCR-T3 ......................................................... 38

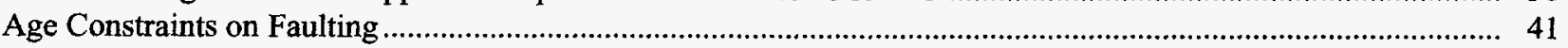

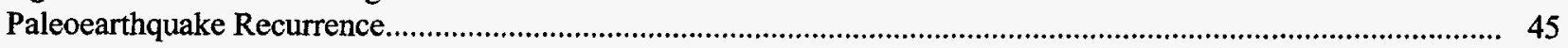

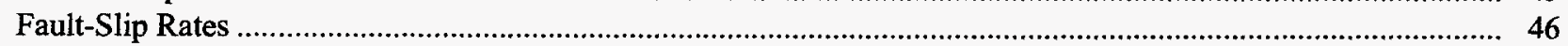

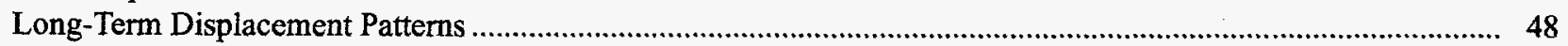

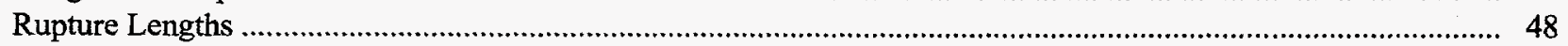

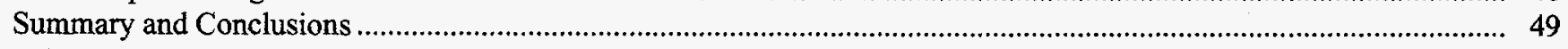

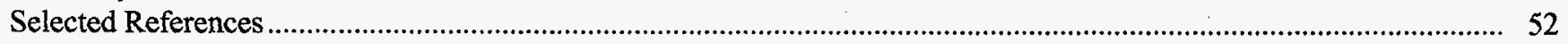

Appendix A .

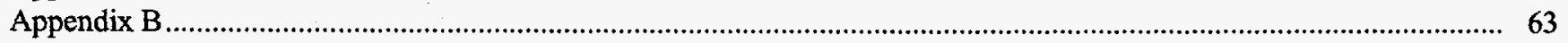




\section{PLATES}

1. Log of part of north and south walls of trenches SCR-T1 and SCR-T3, Stagecoach Road fault, southeast edge of Yucca Mountain, Nye County, Nevada

2. Detailed log of trench SCR-T1, Stagecoach Road fault, southeast edge of Yucca Mountain, Nye County, Nevada

3. Detailed log of trench SCR-T3, Stagecoach Road fault, southeast edge of Yucca Mountain, Nye County, Nevada

\section{FIGURES}

1. Map showing generalized regional geology of Yucca Mountain and vicinity in southwestern Nevada

2. Map showing faults, generalized bedrock outcrop, and trench locations in Yucca Mountain area..........................

3. Photograph showing oblique aerial view looking northeast along Stagecoach Road fault .................................... 6

4. Map showing geologic map of bedrock and surficial deposits in area of trenches on Stagecoach Road fault .......... 7

5. Graph showing topographic profiles of fault scarps developed in surficial deposits across trace of Stagecoach Road fault near trench sites

6. Photographs showing view looking $A$, eastward along trench $\mathrm{SCR}-\mathrm{T} 1 ; B$, north along main Stagecoach Road fault zone in trench SCR-T1; and $C$, north along faults $\mathrm{C}$ and $\mathrm{D}$ of Stagecoach Road fault on upper wall, above bench on north side of trench SCR-T3.

7-10. Maps showing:

7. Portion of $\log$, looking north, of Stagecoach Road fault in trench SCR-T1

8. Portion of log, looking north, of Stagecoach Road fault in trench SCR-T3

9. Simplified interpretive log of portion of trench SCR-T1

10. Simplified interpretive $\log$ of portion of trench SCR-T3

11. Chart illustrating age control and temporal relationships of faulting events in trenches SCR-T1

and SCR-T3.

\section{TABLES}

1. Age boundaries for subdivisions of the Quaternary period

2. Summary characteristics of lithologic units in trenches SCR-TI and SCR-T3...

3. Summary of soil profile descriptions for trenches SCR-T1 and SCR-T3

4. Age estimates based on thermoluminescence data

5. Uranium and thorium isotopic data from carbonates collected from Stagecoach Road fault trenches SCR-T1 and SCR-T3

6. Uranium and thorium isotopic compositions of the authigenic carbonate component for sample aliquots listed in table 5 .

7. Lithostratigraphic correlations and estimated ages of lithologic sequences in trenches SCR-T1 and SCR-T3

8. Criteria used to interpret surface-faulting events in trenches SCR-T1 and SCR-T3.

9. Estimates for apparent displacements per event for the Stagecoach Road fault in trenches SCR-T1 and SCR-T3

10. Age control for composite set of faulting events identified on Stagecoach Road fault in trenches SCR-T1 and SCR-T3

11. Recurrence intervals computed for faulting events on the Stagecoach Road fault using composite timing control from trenches SCR-T1 and SCR-T3

12. Fault slip rates for selected dated horizons on Stagecoach Road fault at trenches SCR-T1 and SCR-T3

13. Summary of paleoseismic parameters for late Quaternary activity on the Stagecoach Road fault 
CONVERSION FACTORS

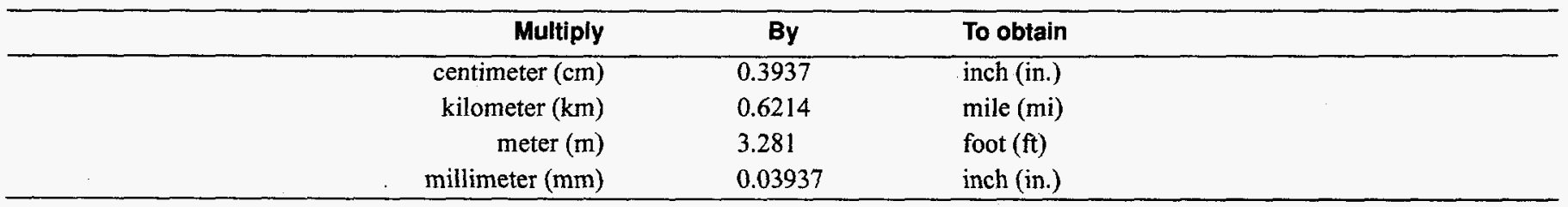

The following terms and abbreviations also are used in this report:

$\mathrm{ka}$

ky

$\mathrm{Ma}$

$\mathrm{mm} / \mathrm{yr}$

Th

TL

U

$\sigma$ kiloannum (thousands of years ago)

thousands of years

million years ago

millimeter per year

thorium

thermoluminescence

uranium

sigma 


\title{
Paleoseismic Investigations of Stagecoach Road Fault, Southeastern Yucca Mountain, Nye County, Nevada
}

\author{
By C.M. Menges, J.A. Oswald, J.A. Coe, S.C. Lundstrom, J.B. Paces, S.A. Mahan, \\ Beth Widmann, and Michele Murray
}

\begin{abstract}
Paleoseismic investigations in two trenches excavated across the Stagecoach Road fault at the southeastern margin of Yucca Mountain provide stratigraphic and structural evidence for multiple paleoearthquakes associated with surface ruptures in the middle to late Quaternary. The fault is topographically expressed by a 1 - to 2.5 -meter-high fault scarp located approximately 0.5 kilometer west and northwest of a low bedrock ridge. Both trenches contain a prominent multi-strand fault zone that strikes north to northeast, dips steeply to the west, and is expressed as a complex, 2- to 5-meter-wide set of carbonate-coated fractures and shears. A wide zone of secondary deformation, including antithetic grabens and eastward backtilting of strata, is present on the hanging-wall block in both trenches.
\end{abstract}

The Stagecoach Road fault displaces a bedrock pediment, which is veneered by a 2- to 3-meter thickness of moderately cemented surficial deposits on the footwall block, against a mixed sequence of unconsolidated to poorly consolidated alluvium, colluvium, and eolian material on the hanging-wall block. Age control for the faulting events is provided by 11 thermoluminescence and $4 \mathrm{U}$-series dates of surficial deposits on the hanging-wall block; combined with soil stratigraphy in the trenches and correlations with a regional Quaternary chronosequence for the Yucca Mountain area. These data indicate a Holocene and late Pleistocene age range for hanging-wall units and a middle to early Pleistocene age range for surficial deposits on the footwall block.

A composite sequence of at least three, preferably five, and possibly seven, individual surface-rupturing events are recorded in hanging wall exposures in both trenches. Criteria used to identify individual faulting events include upward terminations of fractures and shears, incremental increases in the dip of tilted strata, and scarpderived colluvial wedges and related filled fault fissures. The amount of slip associated with these events is difficult to resolve due to absence of correlative units on both sides of the fault, and thus lower and upper bounds for individual and cumulative apparent displacements across the fault have been estimated from the observed thickness of colluvial wedges, adjustments of these wedges to allow for scarp degradation, and the stratigraphic separation of strata between interpreted event horizons. Uncorrected estimates of slip for individual events ranges from 15 to 160 centimeters; preferred values typically are between 30 and 100 centimeters. Corrections for the effect of local backtilting and graben formation at the fault zone reduces the preferred values to a range of 40 to 80 centimeters.

The age of the most recent event is estimated as middle Holocene to latest Pleistocene (5-15 ka), but probably is 10 to 15 thousand years ago $(\mathrm{ka})$. The next two youngest events are considered to be latest Pleistocene (20-30 ka and 30-35 ka) in age. An additional two or three older events may have 
occurred between 40 and $120 \mathrm{ka}$, depending on whether a five- (preferred) or six-event scenario is adopted as a composite faulting chronology for the main sequence of hanging-wall events in both trenches. Several different calculation methods applied to these timing constraints indicate preferred values for individual and average recurrence intervals of 5 to 80 thousand years (ky), and commonly between 5 and $30 \mathrm{ky}$. These recurrence values are not highly sensitive to the specific event scenario selected. The late Quaternary average fault-slip rates are computed for dated horizons by using the net cumulative separations and typically range from 0.01 to 0.07 millimeter per year, with a preferred value of 0.04 millimeter per year. Some evidence for more frequent paleoearthquakes indicates a shortening of recurrence intervals of perhaps 25 to 85 percent for the three most recent events, relative to the poorly constrained older event. This pattern, if real, may reflect a weak temporal clustering of events, although quasi-periodic earthquake recurrence cannot be excluded on the basis of current timing data.

There is no indication of segmentation in the short (4 to 8 kilometers long) main section of the fault zone. However, total rupture lengths are not well constrained because of uncertainties as to whether and how far rupture continues beyond the southwest or northeast ends of the main fault zone. Vertical separation of 99 meters on a late Miocene bedrock tuff near trench SCR-T1 yields long-term displacement rates of 0.02 millimeter per year. The presence of a bedrock pediment on the footwall block and absence of strong soils in the hanging-wall sequence above bedrock suggest a lull in activity in the late Miocene or early Pliocene followed by a fairly continuous interval of late Pliocene-Quaternary faulting.

\section{INTRODUCTION}

Site characterizations for high-level nuclearwaste repositories must address seismic hazard issues such as ground motions and surface displacements associated with earthquakes on faults in and adjacent to the site. Seismic hazard analyses are prerequisite elements of Preclosure and Postclosure Tectonics studies for the potential repository at Yucca Mountain in southwestern Nevada (U.S. Department of Energy, 1988). Seismic source characterization utilizes sitespecific data on Quaternary faulting, including fault length and geometry, the number, amounts, and ages of individual surface displacements, earthquake recurrence intervals, and fault-slip rates (Allen, 1986; Schwartz, 1988; dePolo and Slemmons, 1990; Reiter, 1990). Paleoseismic data on major local and regional faults with known or suspected Quaternary activity in the Yucca Mountain area are being provided by detailed investigations at numerous sites (Sirnonds and others, 1995; Simonds and Whitney, 1993; Menges and others, 1994; Pezzopane and others, 1994).

This report summarizes the results of paleoseismic investigations at two trenches (SCR-T1 and SCR-T3) excavated across the Stagecoach Road (SCR) fault at the southeastern margin of Yucca Mountain (figs. 1, 2). The results of these studies are based on detailed mapping or logging of geologic and structural relationships exposed in trench walls, combined with descriptions of lithologic units, associated soils, and fault-related deformation. The ages of trench deposits are determined directly from geochronologic dating of selected units and soils, supplemented by stratigraphic and soil correlations with other surficial deposits in the Yucca Mountain area. The time boundaries used in this report for subdivision of the Quaternary period are listed in $a+a b$. These data and interpretations are used to identify the number, amounts, timing, and approximate lengths of late to middle Quaternary (less than $200 \mathrm{ka}$ ) surfacefaulting events associated with paleoearthquakes at the trench sites. This displacement history forms the basis for calculating paleoearthquake recurrence intervals and fault-slip rates for the Stagecoach Road fault and allows comparison with fault behavior on other Quaternary faults at or near Yucca Mountain. Other paleoseismic characteristics such as rupture width, rupture area, and magnitudes of paleoearthquakes may be calculated as well, but such calculations are beyond the scope of this study and are planned as part of future seismic hazard analysis. 


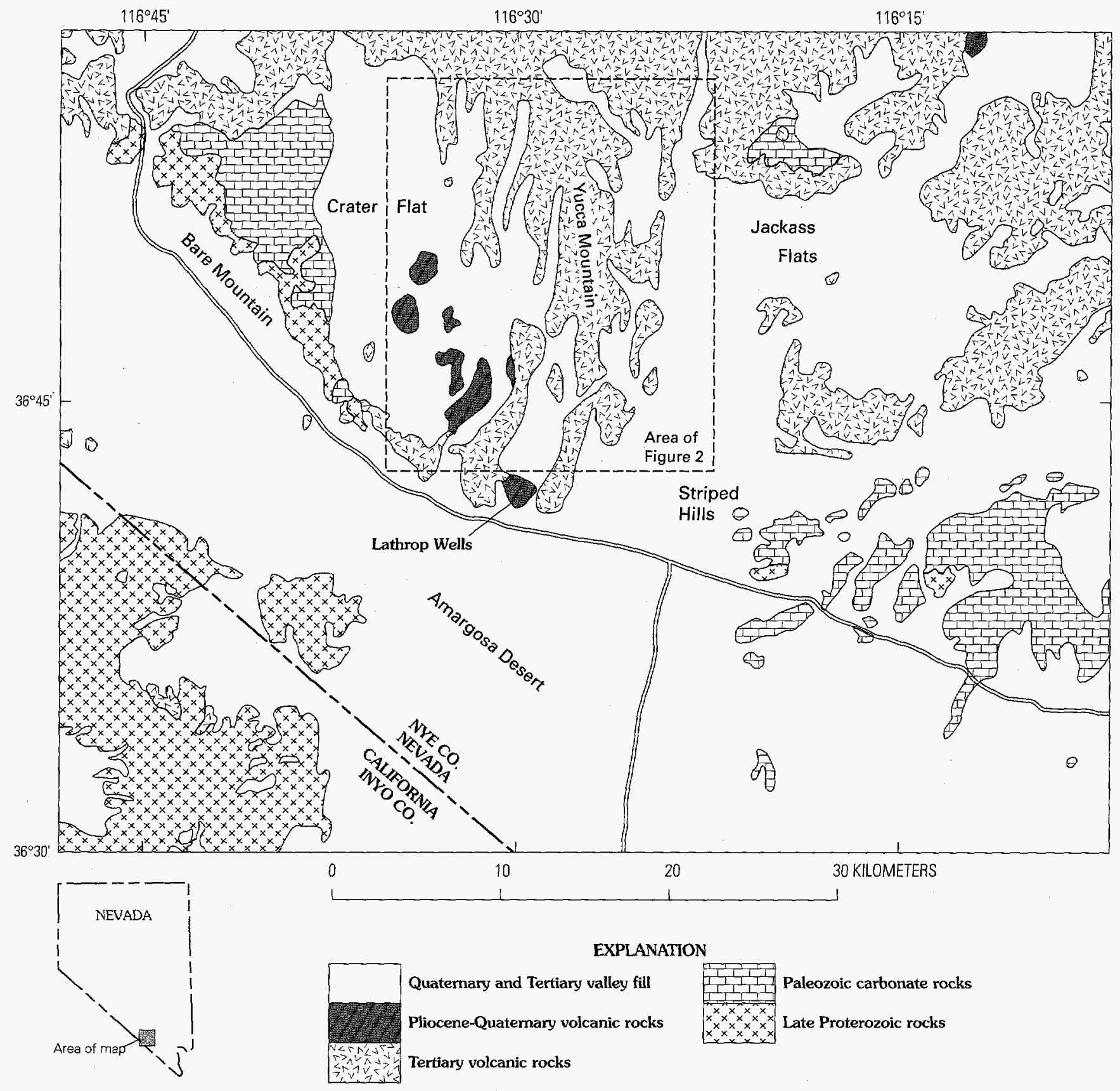

Figure 1. Generalized regional geology of Yucca Mountain and vicinity in southwestern Nevada. 


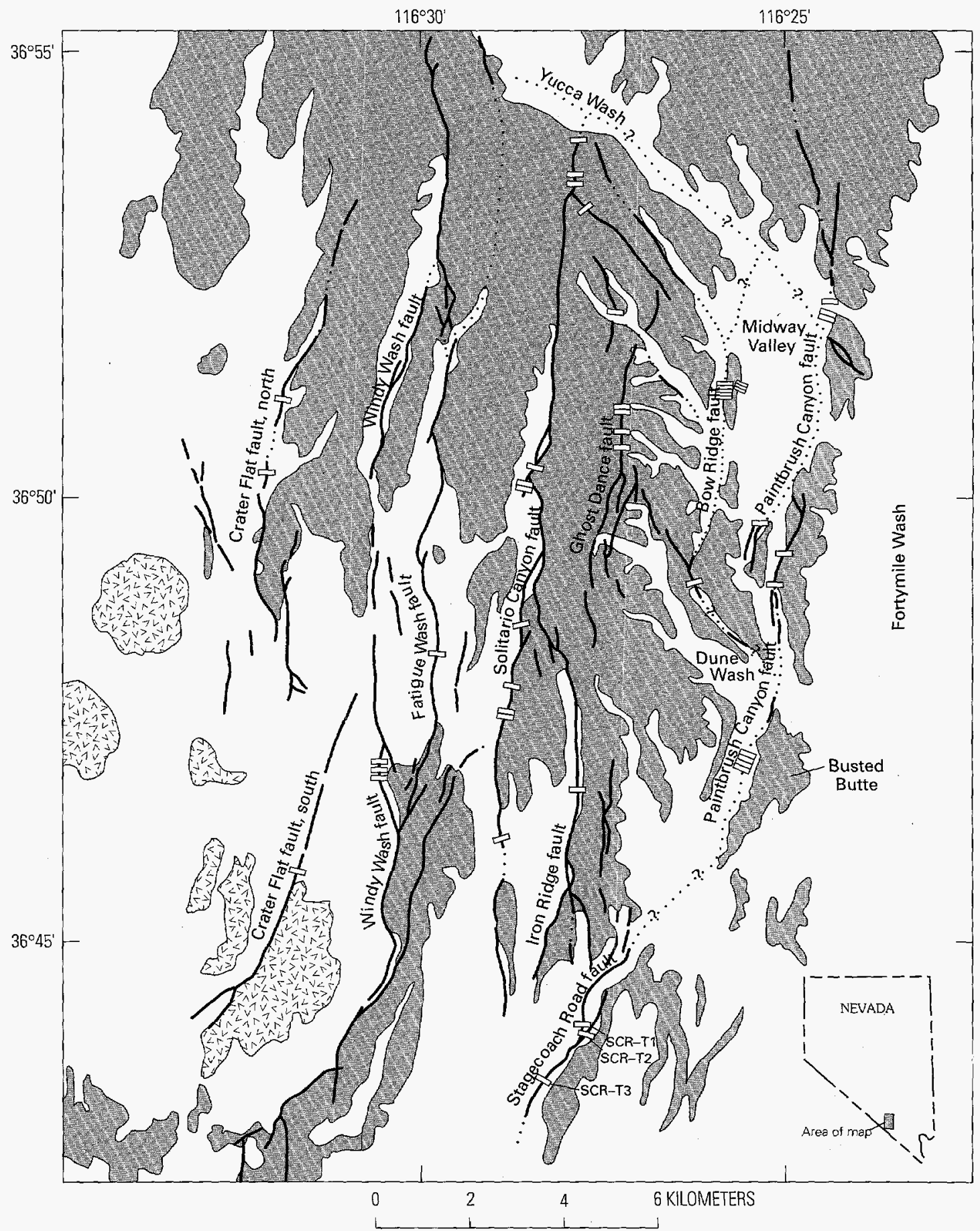

EXPLANATION

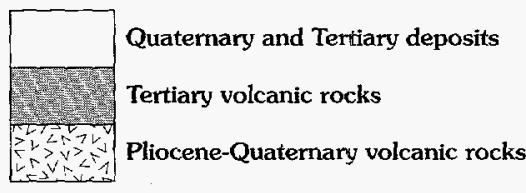

$$
\begin{gathered}
\text { SCR-T3 } \begin{array}{c}
\text { Trench for Quaternary fault activity studies- } \\
\text { Designations shown for trenches discussed in } \\
\text { this report }
\end{array} \\
\text { _..... Fault--Dotted where concealed or approximate, } \\
\text { queried where uncertain }
\end{gathered}
$$

Figure 2. Faults, generalized bedrock outcrop, and trench locations in Yucca Mountain area. 
Table 1. Age boundaries for subdivisions of the Quaternary period

[Modified from Wesling and others (1992) and Harland and others (1990); ka, thousand years ago; Ma, million years ago]

\begin{tabular}{lc}
\hline \multicolumn{1}{c}{ Tlme interval } & Age \\
\hline Late Holocene & Historic-3 ka \\
Middle Holocene & $3-7 \mathrm{ka}$ \\
Early Holocene & $7-10 \mathrm{ka}$ \\
Latest Pleistocene & $10-50 \mathrm{ka}$ \\
Late Pleistocene & $10-128 \mathrm{ka}$ \\
Middle Pleistocene & $128-738 \mathrm{ka}$ \\
Early Pleistocene & $738-1,600 \mathrm{ka}$ \\
Pliocene & $1.6-5.2 \mathrm{Ma}$ \\
\hline
\end{tabular}

\section{GEOLOGIC AND GEOMORPHIC SETTING}

\section{Physiography and Geology of Yucca Mountain Site}

Yucca Mountain consists of a series of northtrending bedrock ridges that are separated by narrow, alluviated valleys (figs. 1,2 ). The altitude of the ridgecrests ranges from 1,250 to $1,710 \mathrm{~m}$, with local relief commonly in the range of 100 to $250 \mathrm{~m}$. These ridges terminate to the north along the northwesttrending valley of Yucca Wash. To the south, the main ridge system becomes more discontinuous and gradually decreases in altitude toward its termination at the northern margin of the alluvial plain of the Amargosa desert. Yucca Mountain is bounded on the west by the alluvial piedmont of the Crater Flat Basin and on the east by the alluvium of Fortymile Wash, the principal drainage for the mountainous terrain to the north.

Bedrock exposed at Yucca Mountain is composed of a thick sequence of middle Miocene (14-11 Ma) silicic pyroclastic flows derived primarily from nearby calderas in the southwestern Nevada volcanic field to the north (Scott and Bonk, 1984; Spengler and Fox, 1989; Carr, 1990; Scott, 1990, 1992; Frizzell and Shulters, 1990; Minor and others, 1993; Sawyer and others, 1994). The main volcanic units at Yucca Mountain are the Topopah Spring, Pah Canyon, Yucca Mountain, and Tiva Canyon Tuffs of the Paintbrush Group, according to the revised stratigraphy of Sawyer and others (1994). Several small basaltic volcanic centers of late Miocene to Quaternary age, including the Lathrop Wells volcanic center, are scattered to the west and south of Yucca Mountain (fig. 1; Carr, 1990; Crowe and others, 1992, 1995). These centers are the source of one or more basalt flows, tephra sheets, and associated cinder cone or spattercone vents.

The middle Miocene volcanic sequence of Yucca Mountain is faulted and tilted gently (less than $10^{\circ}$ ) eastward by a set of predominantly northstriking, normal to normal-oblique faults (figs. 1, 2; Scott and Bonk, 1984; Scott, 1990, 1992). These faults bound a series of tilted fault blocks, with variable amounts of secondary faulting, that form many of the bedrock ridges of Yucca Mountain (Scott and Bonk, 1984; Scott, 1990, 1992). The major blockbounding faults commonly follow the base of westfacing bedrock topographic escarpments that form the eastern margins of the valleys (Scott and Bonk, 1984; Scott, 1992; Frizzell and Shulters, 1990; O'Neill and others, 1992). Most of the major normal faults dip steeply west and have down-to-the-west vertical displacements of 50 to $600 \mathrm{~m}$ that have accumulated since middle Miocene time (Scott and Bonk, 1984; Scott, 1990,1992). Several workers have proposed an additional component of kinematically related clockwise rotation of the fault blocks in Yucca Mountain (Scott, 1990; O'Neill and others, 1992; Fridrich and Price, 1992; Chris Fridrich, U.S. Geological Survey, written commun., 1994). This rotation would result from primarily right-lateral slip on a set of northwest-striking faults and subordinate amounts of left-oblique slip on the north-trending normal faults described above. Regardless, the majority of normal separation and attendant extension occurred coeval with or shortly following middle Miocene volcanism (Scott, 1990; Fridrich and Price, 1992; Chris Fridrich, written commun., 1994). Previous investigations have documented Quaternary displacements on at least five or six of the major normal faults in the Yucca Mountain area (Swadley and others, 1984; O'Neill and others, 1992; Simonds and Whitney, 1993; Simonds and others, 1995).

\section{Geology and Geomorphology of the Stagecoach Road Fault}

The Stagecoach Road fault is a 4- to 8-km-long normal fault that strikes north to northeast and dips $55^{\circ}$ to $85^{\circ}$ northwest (figs. 1, 2; McKay and Sargent, 1970; Maldonado, 1985; Scott, 1990, 1992; Frizzell 
and Shulters, 1990; O'Neill and others, 1992; Simonds and others, 1995). This fault is located 0.1 to $0.5 \mathrm{~km}$ to the northwest of low, isolated bedrock ridges at the southeast end of Yucca Mountain (figs. 2,3). These ridges are composed of the Topopah Spring Tuff and Tiva Canyon Tuff of the Paintbrush Group (McKay and Sargent, 1970; Maldonado, 1985; Scott, 1992). The structural cross sections of Scott (1990) indicate bedrock displacements of as much as $600 \mathrm{~m}$ down to the west across the fault.

The Stagecoach Road fault displaces lower to upper Quaternary surficial deposits with mixed alluvial, colluvial, and eolian characteristics (fig. 4; McKay and Sargent, 1970; Swadley, 1983). An undissected alluvial piedmont, commonly thinly mantled by loess-like eolian deposits, lies to the north and west of the fault. The moderately dissected footwall area east of the fault is underlain by a bedrock erosion surface with a veneer (typically $2-3 \mathrm{~m}$ thick) of alluvium and colluvium locally intermixed with eolian deposits. This mantled bedrock surface abuts an embayed topographic escarpment at the northwestern edge of the bedrock ridge. The base of the ridge in places lies at or slightly east of unnamed subparallel bedrock faults (figs. 2, 4; McKay and Sargent, 1970). There is no evidence for middle or late Quaternary activity on these ridge-bounding faults. The escarpment is mainly erosional in character and appears to bound a bedrock pediment. Thus the Stagecoach Road fault structurally defines the western edge of a low-relief buried bedrock surface of mixed erosional (pediment) and structural origin.

The main trace of the Stagecoach Road fault is well defined geomorphically by a distinct topographic fault scarp. At the two trench sites, scarp heights are 1.1 to $1.2 \mathrm{~m}$ and maximum slope angles are about $8^{\circ}$ (fig. 5). This scarp is developed locally in alluvial surfaces of probable Holocene to late Pleistocene age (fig. 4). The amount and depth of piedmont dissection (as much as $2 \mathrm{~m}$ ) abruptly increases in the uplifted footwall block east of the fault; this gully dissection locally exhumes the fault surface, exposing white carbonate coatings on fractures and shears along the fault trace (fig. 3).

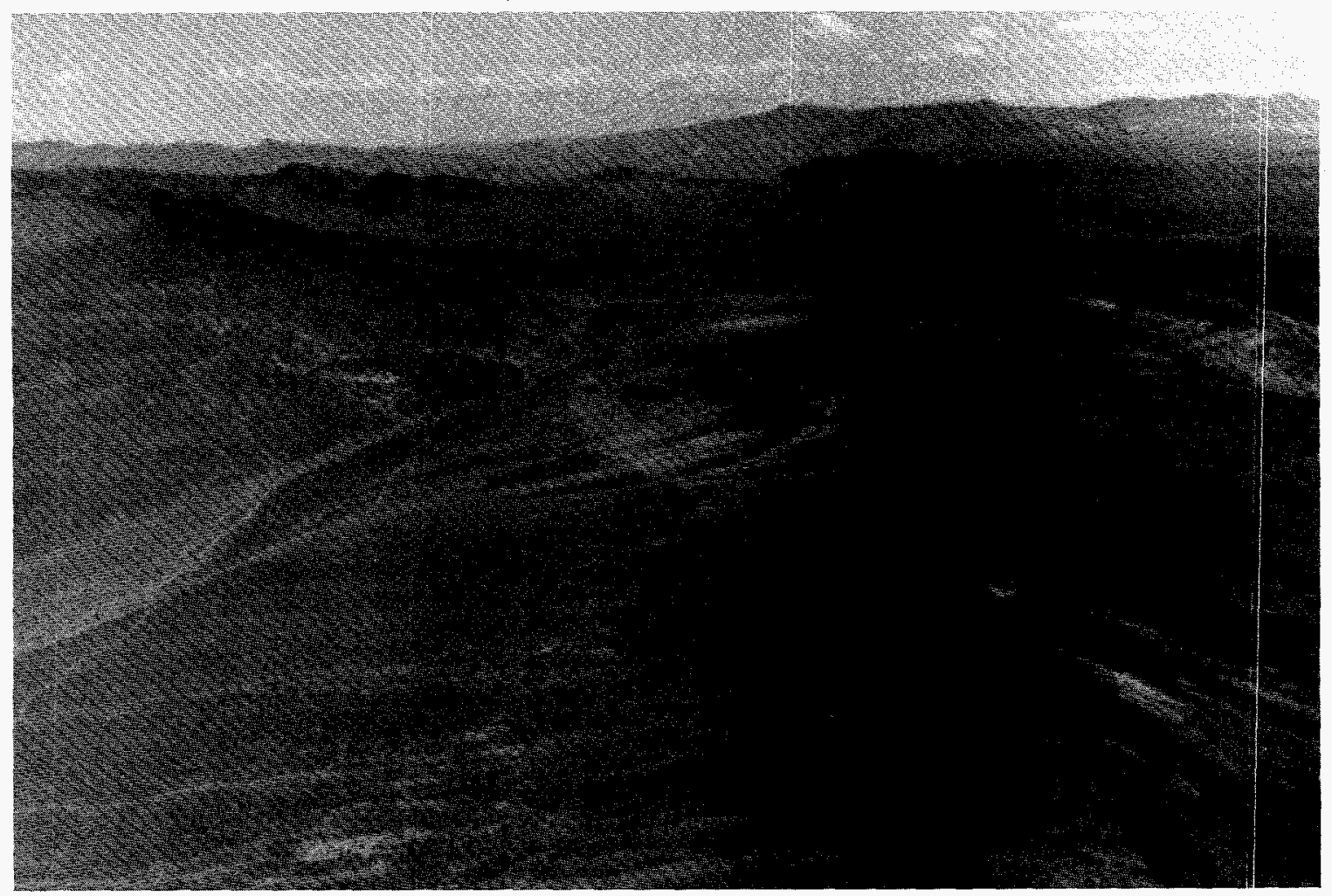

Figure 3. Oblique aerial view looking northeast along Stagecoach Road fault. 


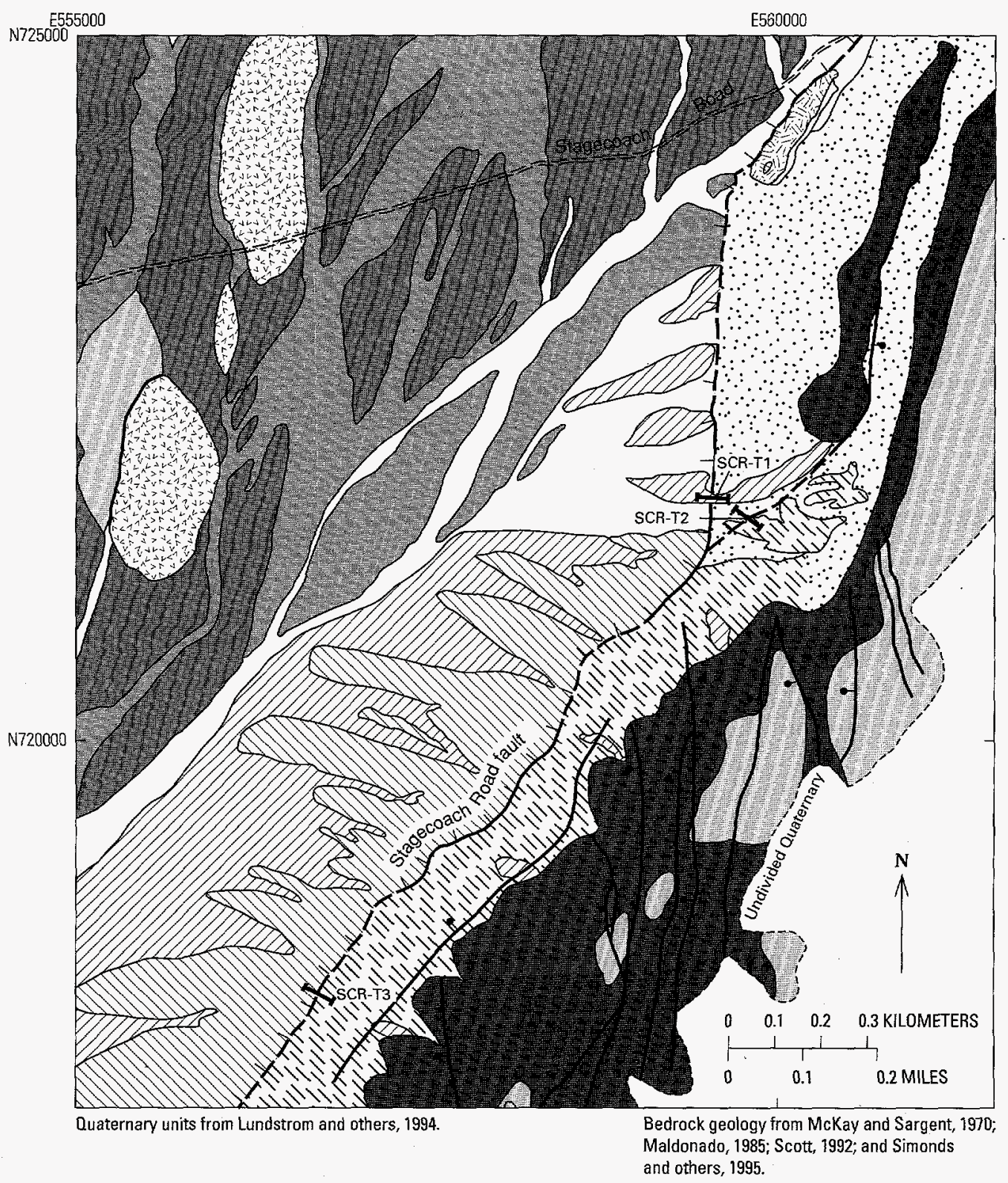

\section{EXPLANATION}

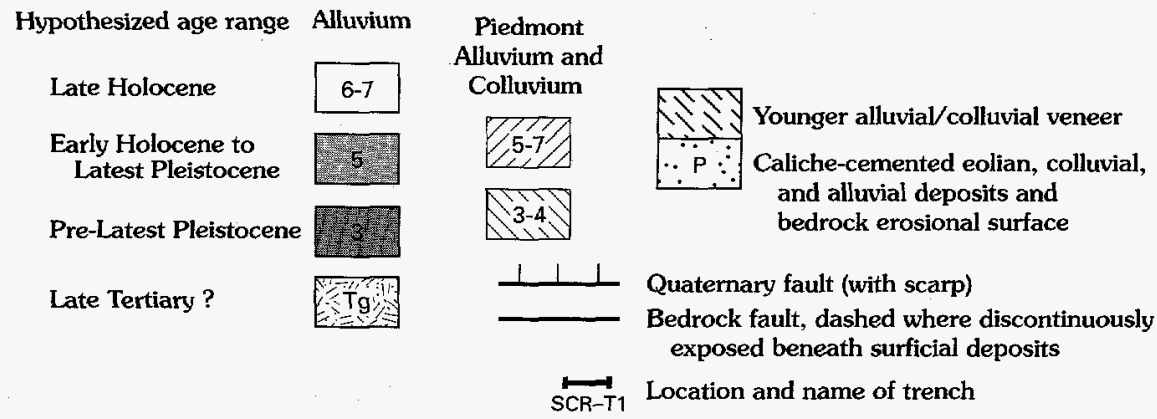

Bedrock (with discontinuous Quaternary colluvial mantle)

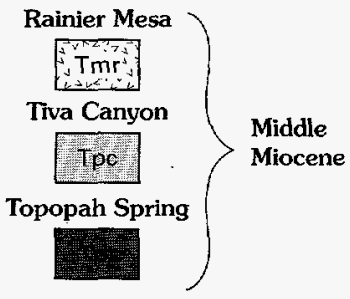

Figure 4. Geologic map of bedrock and surficial deposits in area of trenches on Stagecoach road fault. 


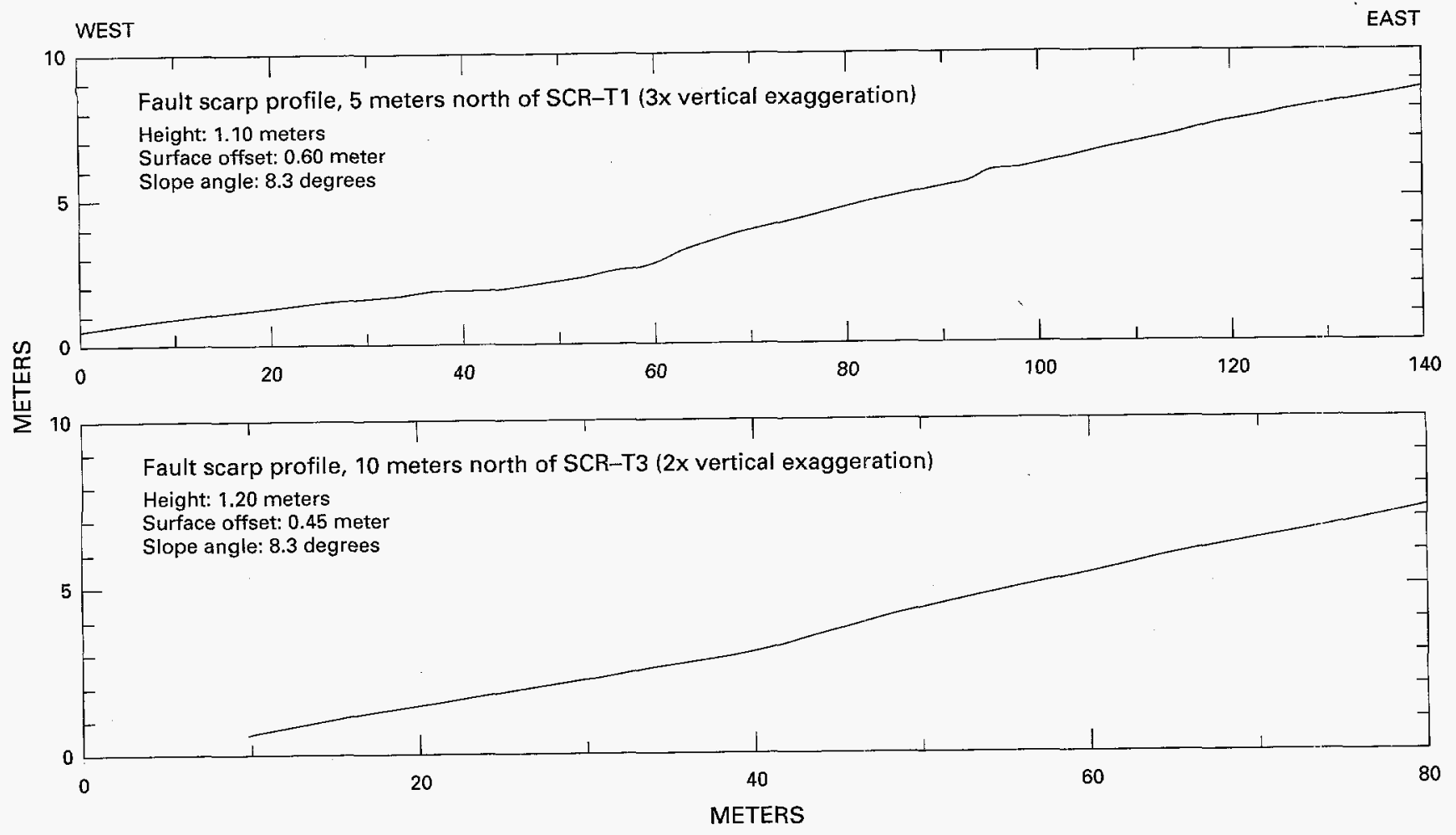

Figure 5. Topographic profiles of fault scarps developed in surficial deposits across trace of Stagecoach Road fault, near trench sites.

The lateral extent of the fault north or south of this bedrock ridge system is poorly constrained from surface exposures. To the southwest, the surface expression of the bedrock fault disappears beneath surficial deposits at the southern end of the main bedrock ridge, several kilometers north of the Lathrop Wells volcanic center. The fault trace appears to terminate to the northeast near a low pass between the ridge and the southern end of Yucca Mountain. The northern continuation of the fault commonly is projected along strike to the northeast to join with the southern end of the Paintbrush Canyon fault at Busted Butte (fig. 2; Maldonado, 1985; Scott, 1990; Frizzell and Shulters, 1990; O'Neill and others, 1992), although no direct surface expression of the fault is evident in upper to middle Quaternary alluvial deposits in the intervening 2 to $3 \mathrm{~km}$ (Simonds and others, 1995). The northeastern projection of the fault is supported by aeromagnetic surveys that show a strong anomaly in the subsurface along this trend. Alternatively, it is possible to connect the northeastern end of the Stagecoach Road fault with the Iron Ridge fault, a southeastern splay of the Solitario Canyon fault (fig. 2; Pezzopane and others, 1994; Simonds and others, 1995).

\section{PALEOSEISMIC INVESTIGATIONS}

\section{Previous Work}

Previous investigations of the Stagecoach Road fault concentrated either on bedrock mapping or on surficial strip mapping designed to document the structural and physiographic expression of the fault, focusing particularly on evidence for Quaternary activity (O'Neill and others, 1992; Simonds and Whitney, 1993; Simonds and others, 1995). This fault was not included in the study of Quaternary faults in the Yucca Mountain area by Swadley and others (1984). No paleoseismic studies have been conducted on the Stagecoach Road fault prior to initiation of the trench investigations reported herein.

\section{Trench Investigations}

Three trenches (SCR-T1, SCR-T2, and SCR-T3) were excavated by backhoe across the Stagecoach Road fault in September 1992. Two of these trenches (SCR-T1 and SCR-T3) are positioned 
where topographic scarps along the main fault trace traverse late Quaternary geomorphic surfaces (fig. 4), and both trenches contain prominent fault zones displacing Quaternary deposits. Trench SCR-T1 is located along the northern part of the fault where it curves into a more northerly to northwesterly direction, whereas trench SCR-T3 lies across the central northeast-striking section of the fault (figs. 2, 4,6 ). Trench SCR-T2 was excavated across a possible secondary fault strand traversing the alluvialmantled pediment east of trench SCR-T1. Trench SCR-T2 exposed an undeformed 2- to $3 \mathrm{~m}$-thick section of early to middle Quaternary alluvium and colluvium above weathered bedrock. No structures or deformation associated with the Stagecoach Road fault are present in the Quaternary units; thus the trench was not logged in detail. Because the late Quaternary paleoseismic record on the main Stagecoach Road fault is best expressed in trenches SCR-T1 and SCR-T3, this report concentrates on results from these two trenches.

Trench SCR-T1 is oriented N. $90^{\circ} \mathrm{E}$. and is approximately $58 \mathrm{~m}$ in length. Trench SCR-T3 is oriented N. $78^{\circ} \mathrm{W}$. and is $50 \mathrm{~m}$ in length. Both trenches are 2- to 3-m wide and reach a maximum depth of $3.5 \mathrm{~m}$. An unusual trench configuration was necessitated by the unconsolidated, unstable materials found in the western (hanging wall) part of both trenches (fig. 6). The eastern (footwall) half of the trenches contains vertical walls on both sides, although the northern wall is benched at midlevel. This benched vertical-wall construction continues along the north side in the western (hanging wall) half of the trenches, but a single vertical wall could not be maintained in the loose material on the south side, and the slope was cut back to a 1:1.5 gradient (fig. 6).

This trench configuration required several different techniques to construct the logs at both trench sites. Fault deformation, lithologic contacts, and soil boundaries were identified and mapped in the field on all walls prior to logging. The vertical south walls in the footwall part of the trenches were logged in the field by using conventional gridding and manual plotting methods (Hatheway and Leighton, 1979; Taylor, written commun.). Logs of the benched northern wall on the hanging-wall half in both trenches were prepared using close-range photogrammetric mapping techniques developed by Fairer and others (1989) and Coe and others (1991). The vertical walls above and below the bench were mapped and photographed individually; individual logs were compiled from annotated photographs of each wall and combined into a single vertical projection plane. This photogrammetric logging was extended to the east of the fault on the north side of both trenches in order to include the upper $1 \mathrm{~m}$ of surficial deposits on the footwall. This was required because the surface soil and deposits on the south side of the trenches were disturbed during excavation. The manual and photogrammetric logs from each trench were merged into a single digital composite log by (a) digitizing the manually plotted field log of the southern footwall, (b) using computer-aided design techniques to project this digital image as if viewed from the south, and (c) moving this projected image of the manually logged south wall below and adjacent to the photogrammetrically compiled north wall of the $\log$ (pls. $1,2,3$ ). Tielines correlate selected features in both sections of the composite logs.

These trench logs are supplemented by field descriptions of lithologic units and soils that are reported in tables 2 and 3 and in appendixes $A$ and $B$. The soil profiles were described at selected locations using the procedures and nomenclature of Birkeland (1984) and Taylor (written commun.), modified from the Soil Survey Staff (1975).

These trench studies were augmented in August 1994, by the drilling of two shallow ( $\leq 105 \mathrm{~m}$ deep) boreholes near Trench SCR-T1. Two adjacent, partly overlapping boreholes (SR-1 and SR-2) were drilled $50 \mathrm{~m}$ west of the fault zone in the trench, and a third borehole (SR-3) is located $75 \mathrm{~m}$ west of the fault. This drilling was designed to sample units present at depth in the hanging wall of the fault. Objectives include determination of the total vertical displacement of bedrock and (or) overlying surficial deposits across the fault, and testing for the presence of carbonate soils in the hanging-wall deposits of comparable strength to the well-developed petrocalcic relict soil on the footwall units (see next section).

\section{Collection and Interpretation of Paleoseismic Data}

Structural relationships between lithologic units, soils, and deformation identified in the trenches are used to characterize surface-faulting events associated with the Stagecoach Road fault, using techniques described by Swan and others (1980), Schwartz 


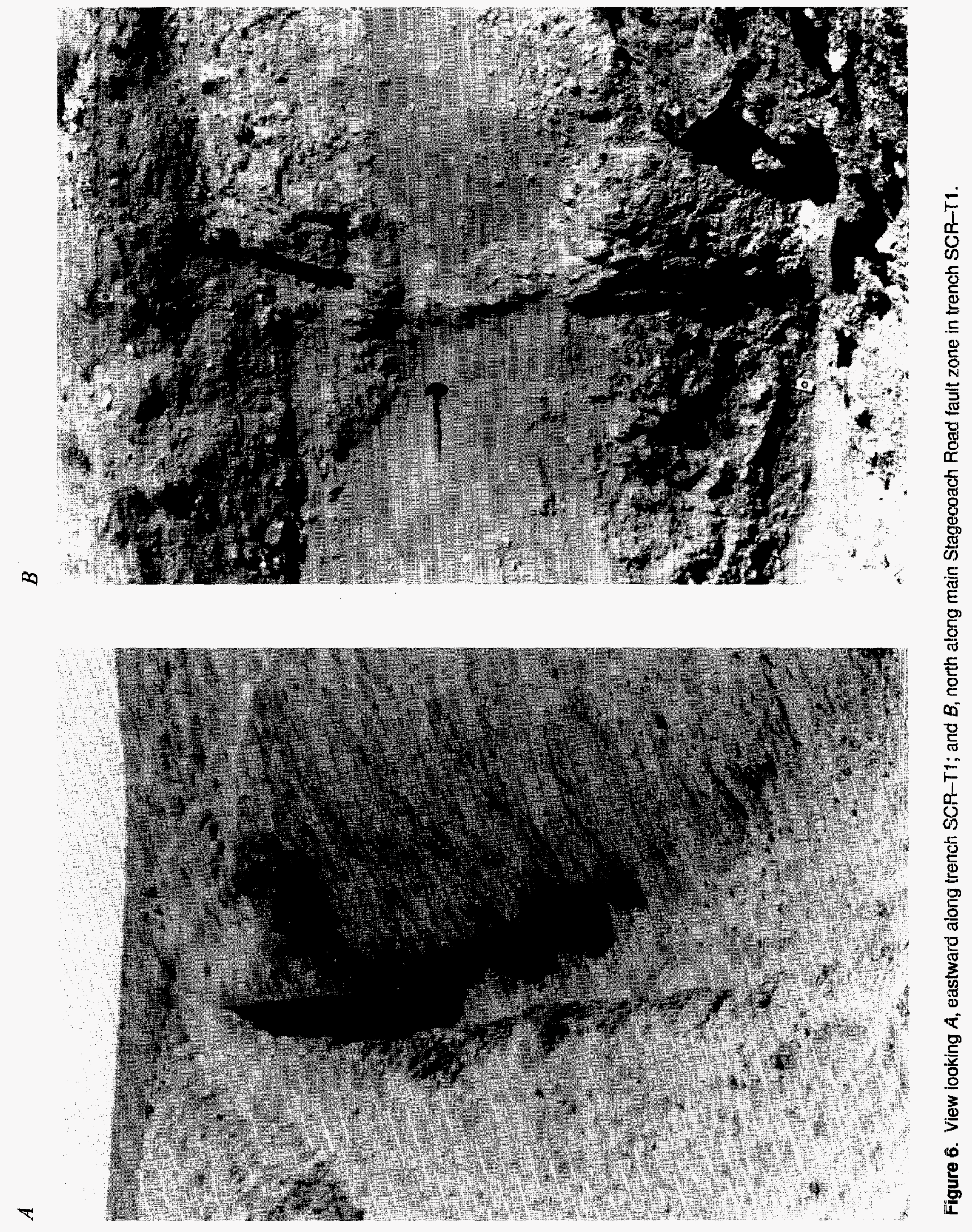




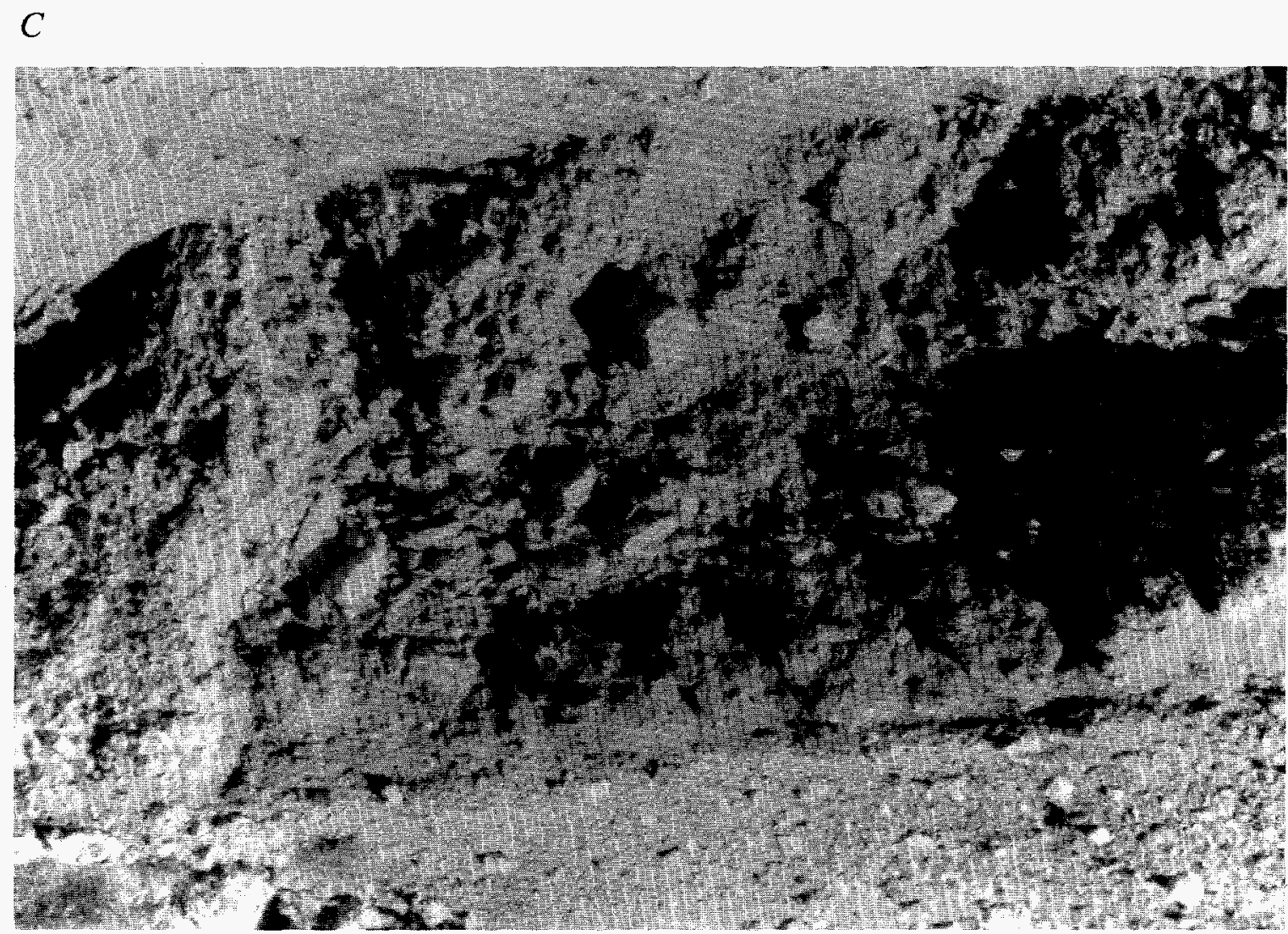

Figure 6.- (Continued) View looking $C$, north along faults $C$ and $D$ of Stagecoach Road fault on upper wall, above bench on north side of trench SCR-T3 (see fig. 8 or pl. 3 ).

(1988), and Nelson (1992). Specific types of paleoseismic data collected are (a) the number and stratigraphic position of interpreted events; (b) measurements of the amounts of apparent displacements per event and net cumulative apparent displacements of dated horizons; and (c) age constraints for faulting events. Behavioral characteristics of the Stagecoach Road fault derived from these data include recurrence intervals, fault-slip rates, and variations in the ages of events. Procedures used for collecting and (or) computing paleoseismic characteristics are described in later sections. All uncertainties in the paleoseismic data are described and incorporated in calculation of fault parameters.

\section{Age Control for Trench Stratigraphy}

Age control for faulted and unfaulted units and soils is a particularly important and difficult problem in paleoseismic investigations at Yucca Mountain. However, a general chronological framework for the Stagecoach Road fault investigations is provided by a composite Quaternary chronosequence of surficial deposits developed in numerous studies in the Yucca Mountain area (Taylor, 1986; Hoover, 1989; Wesling and others, 1992; John Wesling, Geomatrix, Inc., written commun., 1993; Lundstrom and others, 1993, 1994). These chronosequence studies involved mapping of Quaternary allostratigraphic units based on the characteristics of geomorphic surfaces, the relative topographic and geomorphic position of units, and relative degree of soil development. Geochronologic control for these regional Quaternary mapping studies has been provided by several dating techniques, including uranium-trend dating (Rosholt and others, 1985; Swadley and others, 1984), uranium-series disequilibrium (U-Th) dating (Szabo and others, 1981; Muhs and others, 1990; Paces and others, 1993, 1994), thermoluminescence (TL) dating (Whitney and others, 1986; Paces and others, 1994), and limited ${ }^{14} \mathrm{C}$ dating (Swadley and others, 1988). Recent work has shown uranium-trend dating to be an unreliable technique (J. Paces, U.S. Geological Survey, written commun., 1995), thus it has not been used for age determinations in this study. 


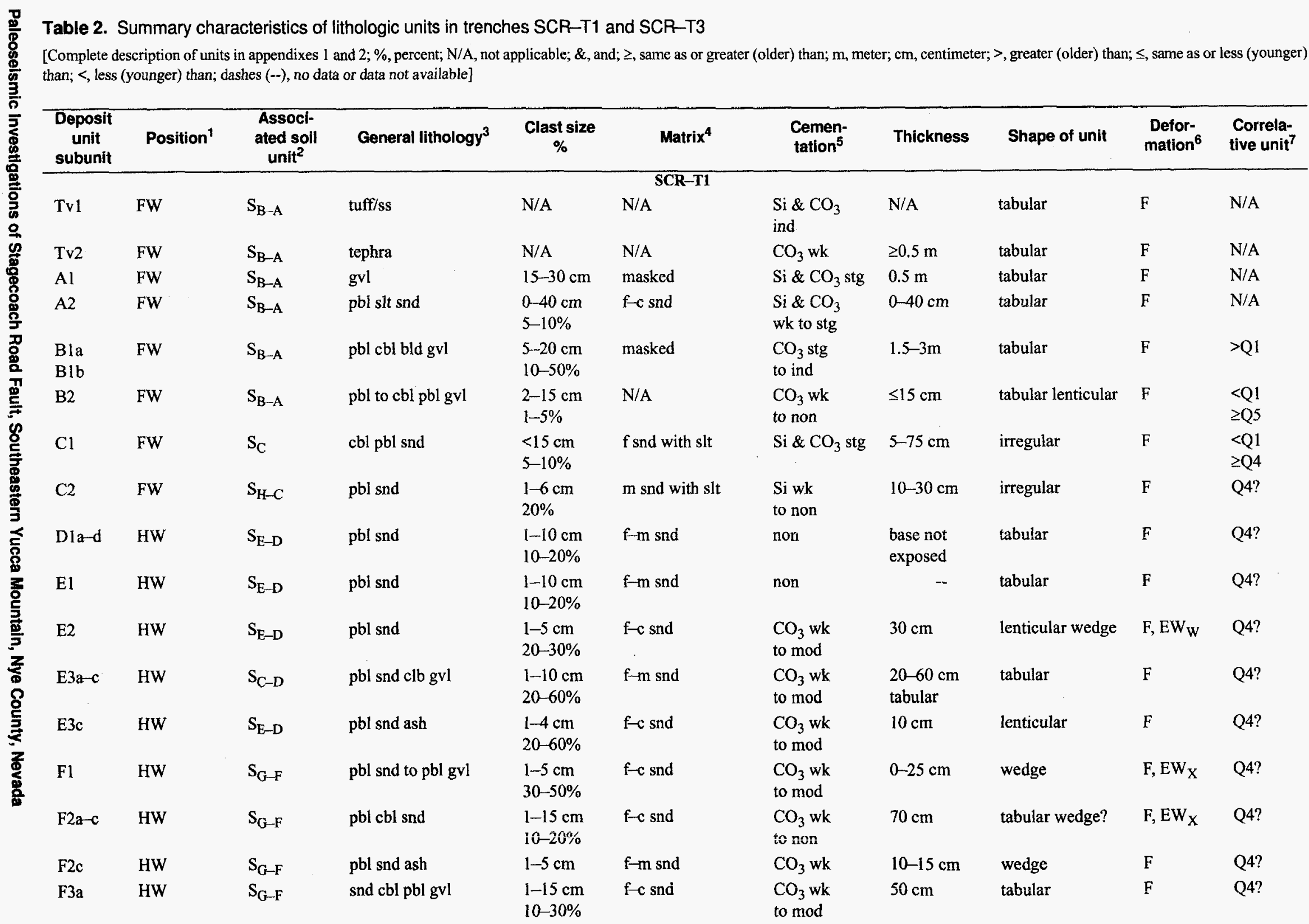


Table 2. Summary characteristics of lithologic units in trenches SCR-T1 and SCR-T3-Continued

[Complete description of units in appendixes 1 and $2 ; \%$, percent; N/A, not applicable; \&, and; $\geq$, same as or greater (older) than; $\mathrm{m}$, meter; cm, centimeter; >, greater (older) than; $\leq$, same as or less (younger) than; <, less (younger) than; dashes (--), no data or data not available]

\begin{tabular}{|c|c|c|c|c|c|c|c|c|c|c|}
\hline $\begin{array}{c}\text { Deposit } \\
\text { unit } \\
\text { subunit }\end{array}$ & Position 1 & $\begin{array}{l}\text { Assocl- } \\
\text { ated soil } \\
\text { unit }{ }^{2}\end{array}$ & General llthology 3 & $\begin{array}{c}\text { Clast size } \\
\%\end{array}$ & Matrlx ${ }^{4}$ & $\begin{array}{l}\text { Cemen- } \\
\text { tation }^{5}\end{array}$ & Thickness & Shape of unit & $\begin{array}{c}\text { Defor- } \\
\text { mation } 6\end{array}$ & $\begin{array}{l}\text { Correla- } \\
\text { tive unit }^{7}\end{array}$ \\
\hline \multicolumn{11}{|c|}{ SCR-T1-Continued } \\
\hline G1 & HW & $\mathrm{S}_{\mathrm{G}-\mathrm{F}}$ & pbl snd & $0-15 \mathrm{~cm}$ & $f-c$ snd & $\mathrm{CO}_{3} \mathrm{wk}$ & $0-15 \mathrm{~cm}$ & fissure wedge & $\mathrm{F}, \mathrm{EW}_{\mathrm{Y}}$ & Q4? \\
\hline$G 2 a-b$ & HW & $\mathrm{S}_{\mathrm{G}-\mathrm{F}}$ & cbl pbl snd & $\begin{array}{l}1-10 \mathrm{~cm} \\
20-50 \%\end{array}$ & $f-c$ snd & non & $20-40 \mathrm{~cm}$ & tabular wedge & $\mathrm{F}, \mathrm{EW}_{\mathrm{Y}}$ & $\mathrm{Q} 4 ?$ \\
\hline G3 & HW & $\mathrm{S}_{\mathrm{G}-\mathrm{F}}$ & $\mathrm{pbl} \mathrm{cbl} \mathrm{snd}$ & $\begin{array}{l}1-15 \mathrm{~cm} \\
20-30 \%\end{array}$ & $f-c$ snd & non & $0-25 \mathrm{~cm}$ & wedge & $\mathrm{EW}_{Z}$ & Q5? \\
\hline G4 & HW & $\mathrm{S}_{\mathrm{G}-\mathrm{F}}$ & cbl pbl snd & $\begin{array}{l}1-12 \mathrm{~cm} \\
20-50 \%\end{array}$ & $f-c$ snd with slt & non & $20-25 \mathrm{~cm}$ & tabular & $\mathrm{U}$ & Q5? \\
\hline G5 & HW & $\mathrm{S}_{\mathrm{G}-\mathrm{F}}$ & $\mathrm{pbl}$ snd & $\begin{array}{l}1-6 \mathrm{~cm} \\
10-20 \%\end{array}$ & $f-c$ snd with slt & non & $0-20 \mathrm{~cm}$ & lenticular & $\mathrm{U}$ & Q5? \\
\hline $\mathrm{H} 1$ & HW\&FW & $\mathrm{S}_{\mathrm{H}}$ & cbl pbl snd & $\begin{array}{l}1-15 \mathrm{~cm} \\
20-30 \%\end{array}$ & f c snd with slt & non & $25-35 \mathrm{~cm}$ & tabular & $\mathrm{U}$ & Q5 \\
\hline $\mathrm{H} 2$ & HW\&FW & $\mathrm{S}_{\mathrm{H}}$ & snd cbl pbl gvl & $\begin{array}{l}1-10 \mathrm{~cm} \\
15-20 \%\end{array}$ & $f-c$ snd with slt & non & $15-30 \mathrm{~cm}$ & tabular & $\mathrm{U}$ & SQ5 \\
\hline $\mathrm{H} 3$ & HW\&FW & $\mathrm{S}_{\mathrm{H}} \mathrm{S}_{\mathrm{H}-\mathrm{C}}$ & cbl pbl snd & $\begin{array}{l}1-15 \mathrm{~cm} \\
10-20 \%\end{array}$ & f-c snd with slt & non & $\leq 20 \mathrm{~cm}$ & tabular & $\mathrm{U}$ & $\leq \mathrm{Q} 5$ \\
\hline $\mathrm{H} 4$ & HW\&FW & $S_{H} S_{H-C}$ & pbl slt snd & $\begin{array}{l}1-10 \mathrm{~cm} \\
10-20 \%\end{array}$ & $\mathrm{f}-\mathrm{m}$ snd with slt & non & $20-30 \mathrm{~cm}$ & tabular & $\mathrm{U}$ & $\leq \mathrm{Q} 5$ \\
\hline \multicolumn{11}{|c|}{ SCR-T3 } \\
\hline $\mathrm{Tv}$ & FW & - & tuff & N/A & N/A & $\begin{array}{l}\mathrm{Si} \& \mathrm{CO}_{3} \\
\text { ind }\end{array}$ & $\begin{array}{l}\text { base not } \\
\text { exposed }\end{array}$ & tabular? & $\mathrm{F}$ & $\begin{array}{l}\text { Tiva } \\
\text { Canyon? }\end{array}$ \\
\hline A1 & FW & $\mathrm{S}_{\mathrm{A}}$ & snd pbl cbl gvl & $\begin{array}{l}1-15 \mathrm{~cm} \\
60-70 \%\end{array}$ & $f-v c$ snd & $\mathrm{CO}_{3}$ wk & $\begin{array}{l}\text { base not } \\
\text { exposed }\end{array}$ & lenticular? & $\mathrm{F}$ & $\geq \mathrm{Q3}$ \\
\hline A2 & FW & $\mathrm{S}_{\mathrm{A}}$ & slt snd pbl cbl bld gvl & $\begin{array}{l}1-30 \mathrm{~cm} \\
60-70 \%\end{array}$ & f-vc snd with slt & $\begin{array}{l}\mathrm{CO}_{3} \mathrm{wk} \\
\text { to mod }\end{array}$ & $\begin{array}{l}\text { base not } \\
\text { exposed }\end{array}$ & irregular & $\mathrm{F}$ & $\geq \mathrm{Q} 3$ \\
\hline A3 & FW & $\mathrm{S}_{\mathrm{A}}$ & snd pbl cbl gvl & $\begin{array}{l}1-20 \mathrm{~cm} \\
60-80 \%\end{array}$ & $f-v c$ snd & $\begin{array}{l}\mathrm{CO}_{3} \bmod \\
\text { to well }\end{array}$ & $\leq 40 \mathrm{~cm}$ & tabular & $\mathrm{F}$ & $\geq \mathrm{Q} 3$ \\
\hline A4 & FW & $\mathrm{S}_{\mathrm{A}}$ & snd pbl cbl gvl & $\begin{array}{l}1-20 \mathrm{~cm} \\
50-70 \%\end{array}$ & f-ve snd & $\begin{array}{l}\mathrm{CO}_{3} \mathrm{wk} \\
\text { to stg }\end{array}$ & $\leq 80 \mathrm{~cm}$ & tabular & $\mathrm{F}$ & $\geq Q 3$ \\
\hline $\mathrm{B} 1 \mathrm{a}-\mathrm{C}$ & FW & $\mathrm{S}_{\mathrm{D}-\mathrm{B}}$ & cbl pbl snd & $\begin{array}{l}1-12 \mathrm{~cm} \\
10-20 \%\end{array}$ & f-ve snd with slt & $\mathrm{CO}_{3} \mathrm{wk}$ & $60-110 \mathrm{~cm}$ & tabular & $\mathrm{F}, \mathrm{EW}_{\mathrm{T}}$ & $\geq Q 3$ \\
\hline $\mathrm{C} 1$ & FW & $\mathrm{S}_{\mathrm{D}-\mathrm{B}}$ & snd pbl cbl gvl & $\begin{array}{l}1-10 \mathrm{~cm} \\
60-80 \%\end{array}$ & $f-m$ snd & $\mathrm{CO}_{3} \bmod$ & $\leq 30 \mathrm{~cm}$ & lenticular & $\mathrm{F}$ & $\geq \mathrm{Q} 3$ \\
\hline $\mathrm{C} 2$ & FW & $\mathrm{S}_{\mathrm{D}-\mathrm{B}}$ & pbl snd & $\begin{array}{l}1-10 \mathrm{~cm} \\
30-40 \%\end{array}$ & f-ve snd & $\mathrm{CO}_{3} w k$ & $\leq 35 \mathrm{~cm}$ & lenticular & $\mathrm{F}$ & $\geq \mathrm{Q} 3$ \\
\hline
\end{tabular}


f Table 2. Summary characteristics of lithologic units in trenches SCR-T1 and SCR-T3-Continued

[Complete description of units in appendixes 1 and 2; \%, percent; N/A, not applicable; \&, and; $\geq$, same as or greater (older) than; m, meter; cm, centimeter; >, greater (older) than; $\leq$, same as or less ( $y$ ounger) than; <, less (younger) than; dashes (--), no data or data not available]

\begin{tabular}{|c|c|c|c|c|c|c|c|c|c|c|}
\hline $\begin{array}{c}\text { Deposit } \\
\text { unit } \\
\text { subunit }\end{array}$ & Position' & $\begin{array}{c}\text { Associ- } \\
\text { ated soil } \\
\text { unit }^{2}\end{array}$ & General lithology 3 & $\begin{array}{c}\text { Clast size } \\
\%\end{array}$ & Matrix ${ }^{4}$ & $\begin{array}{l}\text { Cemen- } \\
\text { tation }^{5}\end{array}$ & Thickness & Shape of unit & $\begin{array}{c}\text { Defor- } \\
\text { mation }^{6}\end{array}$ & $\begin{array}{l}\text { Correla- } \\
\text { tive unit }^{7}\end{array}$ \\
\hline \multicolumn{11}{|c|}{ SCR-T3-Continued } \\
\hline $\mathrm{C} 3$ & FW & $\mathrm{S}_{\mathrm{D} B}$ & snd pbl cbl gvl & $\begin{array}{l}1-10 \mathrm{~cm} \\
40-50 \%\end{array}$ & f c snd & $\begin{array}{l}\mathrm{CO}_{3} \mathrm{mod} \\
\text { to stg }\end{array}$ & $\leq 20 \mathrm{~cm}$ & lenticular & $\mathrm{F}$ & $\geq Q 3$ \\
\hline D1 & FW & $S_{D-B}$ & snd pbl cbl bld gvl & $\begin{array}{l}1-60 \mathrm{~cm} \\
60-80 \%\end{array}$ & $f-v c$ snd & $\begin{array}{l}\mathrm{CO}_{3} \mathrm{mod} \\
\text { to stg }\end{array}$ & $30-100 \mathrm{~cm}$ & tabular & $\mathrm{F}$ & $\geq \mathrm{Q}^{3}$ \\
\hline $\mathrm{D} 2$ & FW & $\mathrm{S}_{\mathrm{D}-\mathrm{B}}$ & pbl snd & $\begin{array}{l}1-5 \mathrm{~cm} \\
20-30 \%\end{array}$ & $f-m$ snd & $\mathrm{CO}_{3}$ wk & $25-30 \mathrm{~cm}$ & tabular & $\mathbf{F}$ & $\geq \mathrm{Q} 3$ \\
\hline D3 & $\mathrm{FW}$ & $\mathrm{S}_{\mathrm{D}-\mathrm{B}}$ & snd pbl cbl gvl & $\begin{array}{l}1-25 \mathrm{~cm} \\
40-70 \%\end{array}$ & $f-c$ snd & $\begin{array}{l}\mathrm{CO}_{3} \bmod \\
\text { to stg }\end{array}$ & $50-60 \mathrm{~cm}$ & tabular & $\mathrm{F}$ & $\geq Q^{3}$ \\
\hline E1 & FW & $\mathrm{S}_{\mathrm{E}}$ & pbl cbl snd & $\begin{array}{l}1-25 \mathrm{~cm} \\
30-50 \%\end{array}$ & f-ve snd & $\mathrm{CO}_{3} \mathrm{stg}$ & $\leq 50 \mathrm{~cm}$ & irregular & $\mathbf{U}$ & $\geq \mathrm{Q} 3$ \\
\hline E2 & FW & $\mathrm{S}_{\mathrm{E}}$ & pbl cbl snd & $\begin{array}{l}1-20 \mathrm{~cm} \\
30-50 \%\end{array}$ & f-ve snd & $\begin{array}{l}\text { Si \& } \mathrm{CO}_{3} \\
\text { wk to stg }\end{array}$ & $\leq 65 \mathrm{~cm}$ & irregular & $\mathrm{U}$ & Q4? \\
\hline $\mathrm{F} 1$ & $\mathrm{HW}$ & $\mathrm{S}_{\mathrm{F}}$ & pbl cbl snd & $\begin{array}{l}1-10 \mathrm{~cm} \\
50-70 \%\end{array}$ & $v f-v c$ snd & $\begin{array}{l}\mathrm{CO}_{3} \mathrm{wk} \\
\text { to mod }\end{array}$ & $\begin{array}{l}\text { base not } \\
\text { exposed }\end{array}$ & tabular & $F$ & Q3-Q4? \\
\hline $\mathrm{F} 2$ & HW & $S_{F}$ & cbl pbl snd gvl & $\begin{array}{l}1-25 \mathrm{~cm} \\
50-70 \%\end{array}$ & $f-c$ snd & $\mathrm{CO}_{3}$ stg & $30-40 \mathrm{~cm}$ & tabular & $F$ & Q3-Q4? \\
\hline$F 3 a-b$ & HW & $\mathrm{S}_{\mathrm{F}}$ & snd cbl pbl gvl & $\begin{array}{l}1-29 \mathrm{cmv} \\
70 \%\end{array}$ & $v f-v c$ snd & $\begin{array}{l}\mathrm{CO}_{3} \text { wk } \\
\text { to stg }\end{array}$ & $80-90 \mathrm{~cm}$ & tabular & $\mathrm{F}$ & Q3-Q4? \\
\hline F4 & HW & $S_{F}$ & gvl snd to snd gvl & $\begin{array}{l}1-30 \mathrm{~cm} \\
40-50 \%\end{array}$ & $v f-v c$ snd with slt & $\begin{array}{l}\mathrm{CO}_{3} \mathrm{wk} \\
\text { to mod }\end{array}$ & $20-30 \mathrm{~cm}$ & tabular & $\mathrm{F}$ & Q3-Q4? \\
\hline F5 & $\mathrm{HW}$ & $S_{F}$ & snd pbl cbl gvl & $\begin{array}{l}1-15 \mathrm{~cm} \\
50-60 \%\end{array}$ & $v f-v e$ snd & $\begin{array}{l}\mathrm{CO}_{3} \mathrm{wk} \\
\text { to } \mathrm{mod}\end{array}$ & $\leq 40 \mathrm{~cm}$ & wedge & $\mathrm{F}, \mathrm{EW}_{\mathrm{V}}$ & Q3-Q4 \\
\hline G1 & HW & $S_{H-G}$ & snd gvl to pbl snd & $\begin{array}{l}1-20 \mathrm{~cm} \\
40-60 \%\end{array}$ & $v f-v e$ snd & $\begin{array}{l}\mathrm{CO}_{3} \mathrm{wk} \\
\text { to mod }\end{array}$ & $15-35 \mathrm{~cm}$ & tabular & $F$ & Q4? \\
\hline G2 & HW & $\mathrm{S}_{\mathrm{H}-\mathrm{G}} \mathrm{S}_{\mathrm{G}}$ & pbl cbl snd & $\begin{array}{l}1-15 \mathrm{~cm} \\
5-60 \%\end{array}$ & $v f-v c$ snd with slt & $\begin{array}{l}\mathrm{CO}_{3} \mathrm{wk} \\
\text { to } \bmod \end{array}$ & $15-50 \mathrm{~cm}$ & tabular & $\mathrm{F}$ & Q4? \\
\hline G3 & HW & $S_{H-G}$ & snd gvl to gvl snd & $\begin{array}{l}1-20 \mathrm{~cm} \\
10-25 \%\end{array}$ & vf-ve snd & $\mathrm{CO}_{3}$ wk & $\leq 45 \mathrm{~cm}$ & wedge? & $\mathrm{F}, \mathrm{EW}_{\mathrm{W}} ?$ & Q4? \\
\hline $\mathrm{G} 4$ & $\mathrm{HW}$ & $\mathrm{S}_{\mathrm{H}-\mathrm{G}} \mathrm{S}_{\mathrm{G}}$ & pbl cbl snd & $\begin{array}{l}1-12 \mathrm{~cm} \\
5-20 \%\end{array}$ & $v f-v c$ snd & $\mathrm{CO}_{3} \mathrm{wk}$ & $25-50 \mathrm{~cm}$ & tabular & $\mathrm{F}$ & Q4? \\
\hline $\mathrm{G} 5 \mathrm{a}-\mathrm{b}$ & HW & $S_{H-G}$ & $\mathrm{pbl} \mathrm{cbl}$ & $1-10 \mathrm{~cm}$ & $v f-v c$ snd & $\mathrm{CO}_{3} \mathrm{wk}$ & $40-50 \mathrm{~cm}$ & tabular & $\mathbf{F}$ & Q4? \\
\hline $\mathrm{H} 1$ & HW & $\mathrm{S}_{\mathrm{H}-\mathrm{G}}$ & $\mathrm{pbl} \mathrm{cbl} \mathrm{snd}$ & $\begin{array}{l}1-20 \mathrm{~cm} \\
5-15 \%\end{array}$ & vf-ve snd & $\begin{array}{l}\mathrm{CO}_{3} \mathrm{wk} \\
\text { to mod }\end{array}$ & $15-40 \mathrm{~cm}$ & tabular & $\mathbf{F}$ & Q4 \\
\hline
\end{tabular}


Table 2. Summary characteristics of lithologic units in trenches SCR-T1 and SCR-T3 - Continued

[Complete description of units in appendixes 1 and 2; \%, percent; N/A, not applicable; \&, and; $\geq$, same as or greater (older) than; $\mathrm{m}$, meter; $\mathrm{cm}$, centimeter; >, greater (older) than; $\leq$, same as or less (younger) than; <, less (younger) than; dashes (--), no data or data not available]

\begin{tabular}{|c|c|c|c|c|c|c|c|c|c|c|}
\hline $\begin{array}{l}\text { Deposit } \\
\text { unit } \\
\text { subunit }\end{array}$ & Position ${ }^{1}$ & $\begin{array}{l}\text { Associ- } \\
\text { ated soil } \\
\text { unit }^{2}\end{array}$ & General lithology 3 & $\begin{array}{c}\text { Clast size } \\
\%\end{array}$ & Matrix ${ }^{4}$ & $\begin{array}{l}\text { Cemen- } \\
\text { tation }^{5}\end{array}$ & Thickness & Shape of unit & $\begin{array}{c}\text { Defor- } \\
\text { mation }^{6}\end{array}$ & $\begin{array}{l}\text { Correla- } \\
\text { tive unit }\end{array}$ \\
\hline \multicolumn{11}{|c|}{ SCR-T3-Continued } \\
\hline $\mathrm{H} 2 \mathrm{a}$ & HW & $\mathrm{S}_{\mathrm{I}-\mathrm{H}}$ & snd gvl to $\mathrm{pbl} \mathrm{cbl} \mathrm{snd}$ & $\begin{array}{l}1-15 \mathrm{~cm} \\
15-20 \%\end{array}$ & $f-v c$ snd & $\begin{array}{l}\mathrm{CO}_{3} \mathrm{wk} \\
\text { to mod }\end{array}$ & $\leq 25 \mathrm{~cm}$ & wedge & $\mathrm{F}, \mathrm{EW}_{\mathrm{X}}$ & Q4 \\
\hline $\mathrm{H} 2 \mathrm{~b}$ & HW & $\mathrm{S}_{\mathrm{I}-\mathrm{H}}$ & pbl cbl snd & $\begin{array}{l}1-25 \mathrm{~cm} \\
15-20 \%\end{array}$ & vf-vc snd & $\mathrm{CO}_{3} \mathrm{wk}$ & $\leq 20 \mathrm{~cm}$ & wedge & $\mathrm{F}, \mathrm{EW}_{\mathrm{X}}$ & Q4 \\
\hline $\mathrm{H} 3 \mathrm{a}-\mathrm{b}$ & HW & $\mathrm{S}_{\mathrm{I}-\mathrm{H}}$ & ash pbl cbl snd & $\begin{array}{l}1-12 \mathrm{~cm} \\
10-15 \%\end{array}$ & $\begin{array}{l}\text { vf-vc snd } \\
<5 \% \text { ash }\end{array}$ & $\mathrm{CO}_{3} \mathrm{wk}$ & $\leq 20 \mathrm{~cm}$ & tabular & $\mathrm{F}$ & Q4 \\
\hline $\mathrm{H} 3 \mathrm{c}$ & HW & $\mathrm{S}_{\mathrm{IH}}$ & pbl cbl snd & $\begin{array}{l}1-10 \mathrm{~cm} \\
10-15 \%\end{array}$ & $v f-v e$ snd with slt & $\begin{array}{l}\mathrm{CO}_{3} \mathrm{wk} \\
\text { to mod }\end{array}$ & $30-50 \mathrm{~cm}$ & tabular & $\mathrm{F}$ & Q4 \\
\hline $11 a-b$ & HW\&FW & $\mathrm{S}_{\mathrm{I}-\mathrm{H}}$ & pbl cbl bld snd & $\begin{array}{l}1-35 \mathrm{~cm} \\
10-30 \%\end{array}$ & vf-ve snd with slt & $\mathrm{CO}_{3} \mathrm{wk}$ & $0-60 \mathrm{~cm}$ & wedge & $\mathrm{F}, \mathrm{EW}_{\mathrm{Y}}$ & Q4-Q5? \\
\hline 12 & HW\&FW & $\mathrm{S}_{\mathrm{I}-\mathrm{H}} \mathrm{S}_{\mathrm{H}-\mathrm{I}}$ & $\mathrm{pbl} \mathrm{cbl} \mathrm{snd}$ & $\begin{array}{l}1-20 \mathrm{~cm} \\
5-25 \%\end{array}$ & $v f-v c$ snd with slt & $\mathrm{CO}_{3} \mathrm{wk}$ & $15-60 \mathrm{~cm}$ & tabular & $\mathrm{F}$ & Q4-Q5 \\
\hline 13 & HW\&FW & $\mathrm{S}_{\mathrm{I}-\mathrm{H}}$ & pbl cbl snd & $\begin{array}{l}1-25 \mathrm{~cm} \\
20-25 \%\end{array}$ & vf-ve snd & non & $\leq 50 \mathrm{~cm}$ & tabular & $\mathrm{F}$ & Q4-Q5? \\
\hline $14 a-b$ & HW\&FW & $\mathrm{S}_{\mathrm{HI}}$ & snd cbl pbl bld gvl & $\begin{array}{l}1-30 \mathrm{~cm} \\
30-40 \%\end{array}$ & $v f-v c$ snd & $\mathrm{CO}_{3} \mathrm{wk}$ & $\leq 45 \mathrm{~cm}$ & $\begin{array}{l}\text { lenticular } \\
\text { wedge fissure }\end{array}$ & $\mathrm{U}, \mathrm{EW}_{\mathrm{Z}}$ & Q4-Q5? \\
\hline $\mathrm{J} 1$ & HW\&FW & $\mathrm{S}_{\mathrm{H}}$ & pbl cbl snd to slt snd & $\begin{array}{l}1-22 \mathrm{~cm} \\
5-40 \%\end{array}$ & vf-vc snd with sit & $\mathrm{CO}_{3} \mathrm{vwk}$ & $15-60 \mathrm{~cm}$ & tabular & $\mathrm{U}$ & Q4-Q5? \\
\hline $\mathrm{J} 2$ & HW\&FW & $\mathrm{S}_{\mathrm{HI}}$ & pbl cbl snd to slt snd & $\begin{array}{l}1-22 \mathrm{~cm} \\
15-25 \%\end{array}$ & vf-vc snd with slt & $\mathrm{CO}_{3} \mathrm{vwk}$ & $\leq 40 \mathrm{~cm}$ & wedge & $\mathrm{U}, \mathrm{EW}_{\mathrm{Z}}$ & Q5 \\
\hline $\mathrm{J3}$ & HW\&FW & $S_{J_{I}}$ & pbl slt snd & $\begin{array}{l}1-6 \mathrm{~cm} \\
5-10 \%\end{array}$ & vf-vc snd with slt & non & $\leq 15 \mathrm{~cm}$ & wedge & $\mathrm{U}, \mathrm{EW}_{\mathrm{Z}}$ & Q5 \\
\hline $\mathrm{J} 4$ & HW\&FW & $S_{\mathrm{HI}_{\mathrm{I}}}$ & pbl cbl slt snd & $\begin{array}{l}1-26 \mathrm{~cm} \\
5-20 \%\end{array}$ & $v f-v c$ snd with sit & non & $10-35 \mathrm{~cm}$ & tabular & $\mathrm{U}$ & $\leq \mathrm{Q} 5$ \\
\hline
\end{tabular}

'Position: $\mathrm{FW}=$ footwall; $\mathrm{HW}=$ hanging wall; HW\&FW=unit in both hanging wall and footwall blocks.

${ }^{2}$ Soil unit in table 3 associated with unit.

${ }^{3}$ General lithology: $s s=$ sandstone; pbl=pebble; cbl=cobble; bld=boulder; snd=sand; slt=silt; gvl=gravel.

${ }^{4}$ Matrix size: $f$, vf=fine, very fine; $m=$ medium; $c$, vc=coarse, very coarse; $s$ nd=sand; slt=silt; masked=matrix obliterated by pedogenic carbonate.

${ }^{5}$ Cementation: $\mathrm{CO}_{3}=$ carbonate; $\mathrm{Si}=$ silica; wk, vwk=weak, very weak; mod=moderate; stg=strong; ind=indurated; non=uncemented.

${ }^{6}$ Deformation: F=faulted; U=unfaulted; EW=event wedge event number

${ }^{7}$ Correlative Quaternary surficial deposit in regional chronosequence (see text). 
क Table 3. Summary of soil profile descriptions for trenches SCR-T1 and SCR-T3

\begin{tabular}{|c|c|c|c|c|c|c|c|c|c|c|c|c|c|c|c|c|c|}
\hline \multirow[b]{2}{*}{$\begin{array}{l}\text { Soil } \\
\text { unit }\end{array}$} & \multirow[b]{2}{*}{ Horizon } & \multirow[b]{2}{*}{$\begin{array}{l}\text { Depth } \\
\text { (cm) }\end{array}$} & \multirow[b]{2}{*}{$\begin{array}{c}\text { Asso- } \\
\text { ciated } \\
\text { unit }\end{array}$} & \multicolumn{2}{|c|}{ Color } & \multirow[b]{2}{*}{$\begin{array}{l}\text { Per- } \\
\text { cent } \\
\text { gravel }\end{array}$} & \multirow[b]{2}{*}{$\begin{array}{l}\text { Tex- } \\
\text { ture }\end{array}$} & \multirow[b]{2}{*}{ Structure ${ }^{3}$} & \multicolumn{2}{|c|}{ Consistence } & \multirow[b]{2}{*}{$-\underset{\text { stage }}{\mathrm{CO}_{3}}$} & \multirow{2}{*}{$\begin{array}{c}\text { Effer- } \\
\text { ves- } \\
\text { cence } \\
(\mathrm{HCl})^{7}\end{array}$} & \multirow[b]{2}{*}{$\begin{array}{l}\text { Cemen- } \\
\text { tation }^{8}\end{array}$} & \multirow{2}{*}{$\begin{array}{l}\text { Hori- } \\
\text { zon } \\
\text { bound- } \\
\text { ary }\end{array}$} & \multirow[b]{2}{*}{ Roots ${ }^{10}$} & \multirow[b]{2}{*}{ Pores ${ }^{11}$} & \multirow[b]{2}{*}{$\begin{array}{l}\text { Rhizo- } \\
\text { liths }^{12}\end{array}$} \\
\hline & & & & Wet & Dry & & & & $\mathrm{Dry}^{4}$ & $\mathrm{Wet}^{5}$ & & & & & & & \\
\hline \multicolumn{18}{|c|}{ SCR-T1 Profile SP I } \\
\hline \multirow[t]{4}{*}{$\mathrm{S}_{\mathrm{H}-\mathrm{C}}$} & $\mathrm{Av}$ & $0-9$ & $\mathrm{H} 4$ & 10YR $3 / 4$ & $10 \mathrm{YR} 6 / 4$ & $\mathrm{~N} / \mathrm{A}$ & 1s-s1 & $1-2 \mathrm{f}-\mathrm{m} \mathrm{pl}$ & so & so po & $\mathrm{N} / \mathrm{A}$ & eo-vse & N.O. & as & If thruout & $\begin{array}{l}3 \text { vf } v \\
\text { thruout }\end{array}$ & N.O. \\
\hline & $\mathrm{Bk}$ & $9-29$ & $\mathrm{H} 4$ & $10 \mathrm{YR} 4 / 4$ & 10YR $7 / 4$ & $\mathrm{~N} / \mathrm{A}$ & sl & $1 \mathrm{f}-\mathrm{m} \mathrm{sbk}$ & so & so po & 1 & $\mathrm{em}$ & cw cont & $\mathrm{cs}$ & $\begin{array}{l}1 \mathrm{vf}-\mathrm{f} \\
\text { thruout }\end{array}$ & $\begin{array}{l}3 \text { vf } v \\
\text { thruout }\end{array}$ & N.O. \\
\hline & $2 \mathrm{Bkql}$ & $29-52$ & $\mathrm{C} 2$ & $7.5 \mathrm{YR} 4 / 6$ & $\begin{array}{l}\text { 7.5YR } \\
6 / 4-6 / 6\end{array}$ & N/A & $\mathrm{sl}$ & $1-3 \mathrm{f}-\mathrm{c}$ sbk & sh-vh & ss ps & 1 & $\mathrm{em}$ & $\begin{array}{c}\mathrm{cw} \mathrm{CO}_{3} \mathrm{cs} \\
\mathrm{SiO}_{2} \\
\text { discont }\end{array}$ & $a b$ & $\begin{array}{l}1 \mathrm{vf}-\mathrm{m} \\
\text { thruout }\end{array}$ & $\begin{array}{l}3 \mathrm{vf} v \\
\text { thruout }\end{array}$ & N.O. \\
\hline & $2 \mathrm{Bkq} 2$ & $52-69$ & $\mathrm{C} 2$ & $7.5 \mathrm{Y} 4 / 4$ & $\begin{array}{l}7.5 \mathrm{YR} \\
6 / 4-7 / 3\end{array}$ & $\approx 20$ & is & $\begin{array}{l}\text { sg-2* vf-c } \\
\text { sbk }\end{array}$ & lo- $\mathrm{h}^{*}$ & so po & 1 & $\mathrm{em}$ & $\begin{array}{c}\mathrm{cw} \mathrm{CO} \mathrm{Cs}_{3} \mathrm{cs} \\
\mathrm{SiO}_{2} \\
\text { discont }\end{array}$ & aw & $1 \mathrm{vf}$ thruout & N.O. & N.O. \\
\hline \multirow[t]{2}{*}{$\mathrm{S}_{\mathrm{C}}$} & 3Bkqmb & $69-94$ & $\mathrm{C} 1$ & 7.5 YR $4 / 4$ & $\begin{array}{l}\text { 7.5YR } \\
6 / 3-6 / 4\end{array}$ & $5-10$ & 1s & $2 \mathrm{~m}-\mathrm{c} \mathrm{sbk}$ & vh & so po & 1 & vse & ci discont & gw & 1 f ped & N.O. & N.O. \\
\hline & \multicolumn{17}{|c|}{ SCR-T1 Profile SP II ${ }^{13}$} \\
\hline \multirow[t]{7}{*}{$\mathrm{S}_{\mathrm{B}-\mathrm{A}}$} & Ap & $0-25$ & -- & - & -- & - & - & -- & m & -- & - & $\ldots$ & $\ldots$ & - & -- & - & -- \\
\hline & $2 \mathrm{Kqmlb}$ & $25-40$ & $\mathrm{~B} 2$ & $\begin{array}{l}10 \mathrm{YR} \\
3 / 2-3\end{array}$ & $\begin{array}{l}\text { 10YR } \\
8 / 1-3\end{array}$ & $1-5$ & is? & $2 \mathrm{c}-\mathrm{vc} \mathrm{pl}$ & eh & so po & IV & ev & ci cont & cs & 1 vf-f lam & N.O. & N.O. \\
\hline & $2 \mathrm{Kqm} 2 \mathrm{~b}$ & $40-115$ & $\mathrm{~B} 1$ & $\begin{array}{l}10 \mathrm{YR} \\
5 / 3-4\end{array}$ & $\begin{array}{l}10 \mathrm{YR} \\
8 / 1-3\end{array}$ & $5-50$ & is? & $\mathrm{m}$ & eh & so po & IV--V & ev & ci cont & gw & $1 \mathrm{vffrac}$ & N.O. & N.O. \\
\hline & $2 \mathrm{Kqm} 3 \mathrm{~b}$ & $115-263$ & $\mathrm{~B} 1$ & $\begin{array}{l}10 \mathrm{YR} \\
6 / 3-4\end{array}$ & $\begin{array}{l}10 \mathrm{YR} \\
8 / 1-3\end{array}$ & $10-20$ & Is & $3 \mathrm{f}-\mathrm{c} \mathrm{sbk}$ & $\mathrm{eh}$ & so po & IV $-V$ & ev & ci cont & gw & $\begin{array}{c}1 \text { vf frac } \\
\text { ped }\end{array}$ & N.O. & N.O. \\
\hline & $\begin{array}{l}2 \mathrm{Bkqb}+ \\
2 \mathrm{Kqmb}\end{array}$ & $263-353$ & $\mathrm{~B} 1$ & $\begin{array}{l}10 \mathrm{YR} \\
5-4 / 4\end{array}$ & $\begin{array}{l}10 \mathrm{YR} \\
8 / 1-3\end{array}$ & $10-20$ & s-sl & $\begin{array}{l}1-3 \mathrm{f}-\mathrm{c} \text { pl a- } \\
\quad \mathbf{s b k}\end{array}$ & eh & so po & III-IV & ev & $\begin{array}{l}\text { cw-ci } \\
\text { discont }\end{array}$ & $a-c-w-i$ & N.O. & $\begin{array}{l}2 \mathrm{~m} \mathrm{i} \\
\mathrm{ped} / \mathrm{nod}\end{array}$ & N.O. \\
\hline & $\mathrm{Ck}$ & $353-?$ & Tv2 & 10YR $6 / 1$ & $10 Y R 7 / 1$ & N/A & tuff & $\mathrm{N} / \mathrm{A}$ & N/A & N/A & III top & em-es & $\operatorname{cs}$ cont & not exp & N.O. & N.O. & N.O. \\
\hline & \multicolumn{17}{|c|}{ SCR-T1 Profile SP III and SP IV } \\
\hline \multirow[t]{4}{*}{$\mathrm{S}_{\mathrm{H}}$} & Avl & $0-7$ & $\mathrm{H} 4$ & IOYR $4 / 4$ & 10YR $7 / 34$ & 10 & $\mathrm{si}$ & $1 \mathrm{~m}-\mathrm{c} \mathrm{pl}$ & so & so po & N/A & $\mathrm{e}$ & N.O. & as & $1 \mathrm{f}-\mathrm{m}$ & $\begin{array}{l}3 \text { vf v } \\
\text { thruout }\end{array}$ & N.O. \\
\hline & Av2 & $7-13$ & $\mathrm{H} 4$ & 10YR $4 / 4$ & 10 YR $5 / 4$ & $10-20$ & s-sl & lf-m pl & so & so po & N/A & e & N.O. & as & $1 \mathrm{f}-\mathrm{m}$ & $\begin{array}{l}3 \mathrm{vf} v \\
\text { thruout }\end{array}$ & N.O. \\
\hline & $2 \mathrm{Bk}$ & $13-40$ & $\mathrm{H} 2$ & 7.5 YR $5 / 4$ & $7.5 \mathrm{YR} 7 / 3$ & $15-20$ & sl & $1 \mathrm{f}-\mathrm{m} \mathrm{sbk}$ & sh & so po & 1 & em & cw discont & $\mathrm{cs}$ & $\begin{array}{l}2 \mathrm{f} \text {-co } \\
\text { thruout }\end{array}$ & N.O. & N.O. \\
\hline & $3 B k$ & $40-74$ & $\mathrm{H} 1$ & $7.5 \mathrm{YR}$ & $7.5 Y R$ & $20-30$ & ls & sg & so & so po & 1 & em & cw discont & $\mathrm{cs}$ & $\begin{array}{l}1 \mathrm{f}-\mathrm{co} \\
\text { thruout }\end{array}$ & N.O. & N.O. \\
\hline \multirow[t]{2}{*}{$s_{G-\bar{z}}$} & 4Bkąb1 & $74-122$ & $\mathrm{G} 2-4$ & $\begin{array}{l}7.5 Y R \quad 4 / 4 \\
6\end{array}$ & 7.5 YR $6 / 46$ & $20-50$ & s! & $2 \mathrm{f}-\mathrm{csbk}$ & $\begin{array}{l}\text { sh-vh* } \\
{ }^{*} \mathrm{SiO}_{2}\end{array}$ & so po & 1 & em & $\begin{array}{c}\mathrm{cw}_{3} \mathrm{cs} \\
\mathrm{SiO}_{2} \\
\text { discont }\end{array}$ & $a w$ & $\begin{array}{l}\text { If } \mathrm{f}-\mathrm{m} \\
\text { thruout }\end{array}$ & N.O. & N.O. \\
\hline & $5 \mathrm{Bkbl}$ & $122-180$ & $\mathrm{~F} 3$ & $\begin{array}{c}7.5 \mathrm{YR} 4 / 4 \\
4 / 3^{*} \\
{ }^{*} \mathrm{CO}_{3}\end{array}$ & $\begin{array}{l}7.5 \mathrm{YR} \\
\quad 6 / 4-7 / 2 * \\
{ }^{*} \mathrm{CO}_{3}\end{array}$ & $10-30$ & sl & $\begin{array}{c}1 \mathrm{f}-\mathrm{c}^{*} \mathrm{sbk} \\
\quad * \mathrm{CO}_{3}\end{array}$ & lo-so & so po & I-III & es-ev & cw discont & $\mathrm{gw}$ & $\begin{array}{l}1 \mathrm{vf}-\mathrm{f} \\
\text { thruout }\end{array}$ & N.O. & $\begin{array}{l}\text { I co } \\
\text { thruout }\end{array}$ \\
\hline
\end{tabular}


Table 3. Summary of soil profile descriptions for trenches SCR-T1 and SCR-T3 - Continued

[See log for locations of soil profiles; $\mathrm{cm}$, centimeter; $\%$, percent; $\mathrm{CO}_{3}$, carbonate; $\mathrm{HCl}$, cold dilute hydrochloric acid; $\mathrm{N} / \mathrm{A}$, not applicable; N.O., not observed; $\approx$, approximately; ${ }^{*}$, soil property found locally; dashes (--), no data; rizo, rhizolith]

\begin{tabular}{|c|c|c|c|c|c|c|c|c|c|c|c|c|c|c|c|c|c|}
\hline \multirow[b]{2}{*}{$\begin{array}{l}\text { Soil } \\
\text { unit }\end{array}$} & \multirow[b]{2}{*}{ Horizon } & \multirow[b]{2}{*}{$\begin{array}{l}\text { Depth } \\
\text { (cm) }\end{array}$} & \multirow{2}{*}{$\begin{array}{l}\text { Asso- } \\
\text { ciated } \\
\text { unit }\end{array}$} & \multicolumn{2}{|c|}{ Color' } & \multirow{2}{*}{$\begin{array}{l}\text { Per- } \\
\text { cent } \\
\text { gravel }\end{array}$} & \multirow[b]{2}{*}{$\begin{array}{l}\text { Tex- } \\
\text { ture }\end{array}$} & \multirow[b]{2}{*}{ Structure $^{3}$} & \multicolumn{2}{|c|}{ Consistence } & \multirow[b]{2}{*}{$\begin{array}{c}\mathrm{CO}_{3} \\
\text { stage }^{6}\end{array}$} & \multirow{2}{*}{$\begin{array}{c}\text { Effer- } \\
\text { ves- } \\
\text { cence } \\
(\mathrm{HCl})^{7}\end{array}$} & \multirow[b]{2}{*}{$\begin{array}{l}\text { Cemen- } \\
\text { tation }^{8}\end{array}$} & \multirow{2}{*}{$\begin{array}{l}\text { Hori- } \\
\text { zon } \\
\text { bound- } \\
\text { ary }\end{array}$} & \multirow[b]{2}{*}{ Roots ${ }^{10}$} & \multirow[b]{2}{*}{ Pores ${ }^{11}$} & \multirow[b]{2}{*}{$\begin{array}{l}\text { Rhizo- } \\
\text { liths }{ }^{12}\end{array}$} \\
\hline & & & & Wet & Dry & & & & Dry ${ }^{4}$ & Wet ${ }^{5}$ & & & & & & & \\
\hline & \multicolumn{17}{|c|}{ SCR-T1 Profile SP III and SP IV-Continued } \\
\hline & $6 \mathrm{Bkb} 1$ & $180-230$ & $\mathrm{~F} 2$ & $\begin{array}{l}7.5 Y R \text { Y/2- } \\
3\end{array}$ & $\begin{array}{l}7.5 \mathrm{YR} \\
6 / 3-7 / 2 * \\
{ }^{*} \mathrm{CO}_{3}\end{array}$ & $10-20$ & s-s! & $\begin{array}{l}\text { sg-1* f-c } \\
\quad \text { sbk * rizo }\end{array}$ & $\begin{array}{l}\text { lo-sh* } \\
*_{\text {rizo }}\end{array}$ & so po & $\mathrm{I}-\mathrm{II}$ & em-es & cw discont & $\mathrm{cw}$ & N.O. & N.O. & $\begin{array}{l}3 \mathrm{f}-\mathrm{co} \\
\text { thruout }\end{array}$ \\
\hline \multirow[t]{2}{*}{$\mathrm{S}_{\mathrm{E}-\mathrm{D}}$} & 7Bkb2 & $230-270$ & E1-3 & $\begin{array}{l}\text { 7.5YR } 5 / 3- \\
4 / 3\end{array}$ & $\begin{array}{l}7.5 \mathrm{YR} \\
7 / 2-8 / 2 * \\
{ }^{*} \mathrm{CO}_{3}\end{array}$ & $20-60$ & s-sl & $\begin{array}{l}1-2 \mathrm{f}-\mathrm{c} \text { abk- } \\
\text { sbk }\end{array}$ & h & so po & II-III & ev & cs discont & $\mathrm{cw}$ & N.O. & N.O. & $\begin{array}{l}3 \text { f-co } \\
\text { thruout }\end{array}$ \\
\hline & $8 \mathrm{Bkb} 2$ & $270-?$ & D1 & $10 \mathrm{YR} 4 / 4$ & $\begin{array}{l}10 \mathrm{YR} \\
7 / 3-6 / 4\end{array}$ & $10-20$ & is & $1 \mathrm{f}-\mathrm{m} \mathrm{sbk}$ & sh & so po & $\mathrm{I}-\mathrm{II}$ & e-es & cw discont & not exp & N.O. & N.O. & $\begin{array}{l}2 \mathrm{f}-\mathrm{co} \\
\text { thruout }\end{array}$ \\
\hline \multicolumn{18}{|c|}{ SCR-T3 Profile SP I } \\
\hline \multirow[t]{4}{*}{$\mathrm{S}_{\mathrm{IE}}$} & Avl & $0-6$ & $\mathrm{~J} 1-4$ & $10 Y R 4 / 6$ & 10YR $7 / 4$ & $5-20$ & sl & $1 \mathrm{f}-\mathrm{c} \mathrm{pl}$ & so & so po & $\mathrm{N} / \mathrm{A}$ & vse & N.O. & as & $\begin{array}{l}1 \mathrm{vf-f} \\
\text { thruout }\end{array}$ & $\begin{array}{l}3 \text { vf v } \\
\text { thruout }\end{array}$ & N.O. \\
\hline & Av2 & $6-20$ & $\mathrm{~J} 1-4$ & I0YR $4 / 4$ & 10YR $7 / 4$ & $5-10$ & slt-ls & $1 \mathrm{c} \mathrm{pl}$ & $\mathrm{sh}$ & ss ps & $\mathrm{N} / \mathrm{A}$ & e & N.O. & as & $\begin{array}{l}1 \mathrm{vf-m} \\
\text { thruout }\end{array}$ & $\begin{array}{l}2 \mathrm{vf} v \\
\text { thruout }\end{array}$ & N.O. \\
\hline & 2Bk & $20-40$ & $\mathrm{~J} 1-4$ & 7.5 YR 4/6 & $7.5 Y R 6 / 4$ & $15-30$ & sit 1 & $2 \mathrm{c} \mathrm{sbk}$ & sh & ss ps & 1 & $\mathrm{em}$ & $\mathrm{cw}$ discont & a w & $\begin{array}{l}1 \text { vf-f } \\
\text { thruout }\end{array}$ & $\begin{array}{c}1 \mathrm{vf}-\mathrm{f} \mathrm{v}-\mathrm{i} \\
\text { scatt }\end{array}$ & N.O. \\
\hline & 3Bkqm & $40-73$ & E2 & $7.5 \mathrm{YR} 4 / 4$ & 7.5 YR $6 / 4$ & $20-50$ & sl & $3 \mathrm{f}-\mathrm{c}$ sbk & $\mathrm{h}$ & so po & 1 & vse-e & $\begin{array}{l}\text { cs } \mathrm{SiO}_{2} \\
\text { discont }\end{array}$ & as & $\begin{array}{l}1 \mathrm{vf-f} \\
\text { thruout }\end{array}$ & $\begin{array}{l}2-3 \mathrm{~m} \mathrm{i} \\
\text { ped cls }\end{array}$ & N.O. \\
\hline $\mathrm{S}_{\mathrm{E}}$ & 4Kqmb 1 & $73-100$ & $\mathrm{E} 1$ & $7.5 Y R 6 / 4$ & $7.5 Y R 8 / 2-3$ & $30-50$ & Is & $2 \mathrm{~m}-\mathrm{c} \mathrm{sbk}$ & eh & so po & III-IV & es & ci cont & $\mathrm{c} \mathrm{i}$ & N.O. & N.O. & N.O. \\
\hline \multirow[t]{2}{*}{$\mathrm{S}_{\mathrm{D}-\mathrm{B}}$} & $5 \mathrm{Kqmb} 2$ & $100-116$ & D3 & 7.5 YR $6 / 3$ & 7.5YR 8/2.1 & 70 & Is & $\mathrm{m}$ & eh & so po & III & es & ci cont & a-ci & N.O. & N.O. & N.O. \\
\hline & \multicolumn{17}{|c|}{ SCR-T3 Profile SP II } \\
\hline \multirow[t]{2}{*}{$S_{\text {JE }}$} & Ap & $0-20$ & $J 1-4$ & -- & -- & -- & -- & -- & - & -- & -- & -- & -- & - & -- & -- & -- \\
\hline & $2 \mathrm{Bkq}$ & $20-35$ & E2 & 7.5 YR 5/6 & $7.5 Y R \quad 7 / 4-6$ & $20-30$ & sl & $2-3 \mathrm{f}-\mathrm{c} \mathrm{sbk}$ & $\mathrm{sh}-\mathrm{h}$ & so po & 1 & vse & $\begin{array}{l}\text { cw-cs } \\
\quad \text { discont }\end{array}$ & a w-i & $\begin{array}{l}1 \text { vf-c } \\
\text { thruout }\end{array}$ & $\begin{array}{c}1-2 \text { vf-f v-i } \\
\text { ped cls }\end{array}$ & N.O. \\
\hline \multirow[t]{6}{*}{$\mathrm{S}_{\mathrm{D}-\mathrm{B}}$} & 3Kqmb1 & $35-75$ & D3 & 7.5 YR $4 / 6$ & $\begin{array}{l}\text { 7.5YR } \\
8 / 2-7 / 4\end{array}$ & $20-40$ & s-1s & $\mathrm{m}$ & eh & so po & III-IV & ev & ci cont & $\mathrm{cw}$ & N.O. & N.O. & N.O. \\
\hline & $4 \mathrm{Kqbl}$ & $75-105$ & D1 & $10 \mathrm{YR} 5 / 3$ & $\begin{array}{l}10 \mathrm{YR} \\
7 / 3-8 / 2\end{array}$ & $40-70$ & s & I f-m sbk & h-vh & so po & II-III & es & es discont & c-g w & $\begin{array}{l}\text { I vf-f } \\
\text { thruout }\end{array}$ & $\begin{array}{c}2 \mathrm{~m} \text { i ped } \\
\text { clast }\end{array}$ & $\underset{\text { top }}{1 \mathrm{f}-\mathrm{m} \text { at }}$ \\
\hline & $5 \mathrm{Bkbl}$ & $105-200$ & $\mathrm{Bi}$ & $10 \mathrm{YR} 5 / 3$ & $\begin{array}{l}10 \mathrm{YR} 8 / 2-3 \\
\left(\mathrm{CO}_{3}\right)-7 / 3\end{array}$ & $10-20$ & s & sg-1 f-c sbk & so-h & so po & I-II & $\mathrm{em}$ & cw discont & aw & l f scatt & $\begin{array}{l}1 \mathrm{f}-\mathrm{mi} \\
\text { thruout }\end{array}$ & $\begin{array}{l}3 \text { vf-co } \\
\text { thruout }\end{array}$ \\
\hline & $6 \mathrm{Kq} 1 \mathrm{~b} 2$ & $200-226$ & $\mathrm{~A} 4$ & $\begin{array}{l}7.5 \text { YR } 5 / 4 \\
3\end{array}$ & $\begin{array}{c}7.5 \mathrm{YR} 7 / 3 \text { to } \\
8 / 2-3\end{array}$ & $50-70$ & sl-ls & $1 \mathrm{~m}-\mathrm{c} \mathrm{sbk}$ & h-vh & so po & III & es & cs-ci cont & $\mathrm{cw}$ & N.O. & $\begin{array}{l}1 \mathrm{f} \mathrm{i} \mathrm{ped} \\
\text { clast }\end{array}$ & $\begin{array}{l}1 \mathrm{f}-\mathrm{m} \\
\text { thruout }\end{array}$ \\
\hline & $6 \mathrm{Kq} 2 \mathrm{~b} 2$ & $226-245$ & $\mathrm{~A} 4$ & $10 Y R 5 / 4$ & 10YR $8 / 3-7 / 3$ & $50-70$ & si & $1 \mathrm{f}-\mathrm{m} \mathrm{sbk}$ & vh & so po & $\mathrm{II}^{+}-\mathrm{III}$ & es & $\begin{array}{l}\text { cs-ci } \\
\text { discont }\end{array}$ & gw & If scatt & $\begin{array}{l}1 \mathrm{f} \mathrm{i} \mathrm{ped} \\
\text { clast }\end{array}$ & N.O. \\
\hline & 7Bkqb2 & $245-?$ & $\mathrm{~A} 2$ & 7.5 YR $5 / 4$ & 7.5 YR $8 / 3$ & $60-70$ & $\mathbf{s}$ & $1 \mathrm{c} \mathrm{sbk}$ & $\mathrm{h}$ & so po & II-III & es & cs discont & not exp & N.O. & $\begin{array}{l}1 \mathrm{f} \mathrm{i} \mathrm{ped} \\
\text { clast }\end{array}$ & N.O. \\
\hline
\end{tabular}


$\vec{\infty}$ Table 3. Summary of soil profile descriptions for trenches SCR-T1 and SCR-T3-Continued

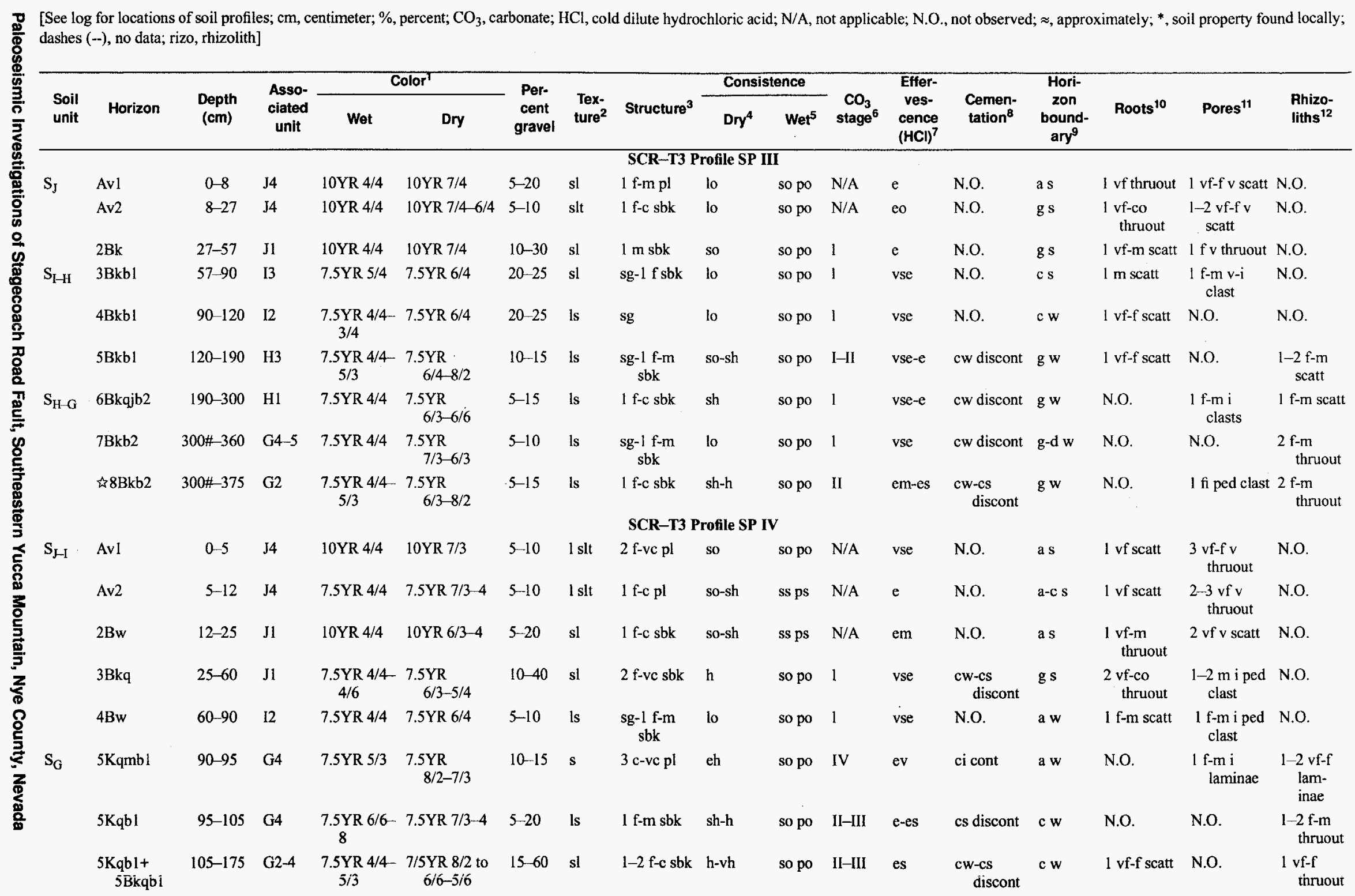


Table 3. Summary of soil profile descriptions for trenches SCR-T1 and SCR-T3-Continued

[See log for locations of soil profiles; $\mathrm{cm}$, centimeter; \%, percent; $\mathrm{CO}_{3}$, carbonate; $\mathrm{HCl}$, cold dilute hydrochloric acid; $\mathrm{N} / \mathrm{A}$, not applicable; N.O., not observed; $\approx$, approximately; *, soil property found locally; dashes (--), no data; rizo, rhizolith]

\begin{tabular}{|c|c|c|c|c|c|c|c|c|c|c|c|c|c|c|c|c|c|}
\hline \multirow[b]{2}{*}{$\begin{array}{l}\text { Soil } \\
\text { unit }\end{array}$} & \multirow[b]{2}{*}{ Horizon } & \multirow[b]{2}{*}{$\begin{array}{l}\text { Depth } \\
\text { (cm) }\end{array}$} & \multirow{2}{*}{$\begin{array}{c}\text { Asso- } \\
\text { ciated } \\
\text { unit }\end{array}$} & \multicolumn{2}{|c|}{ Color ${ }^{1}$} & \multirow{2}{*}{$\begin{array}{c}\text { Per- } \\
\text { cent } \\
\text { gravel }\end{array}$} & \multirow[b]{2}{*}{$\begin{array}{l}\text { Tex- } \\
\text { ture }\end{array}$} & \multirow[b]{2}{*}{ Structure ${ }^{3}$} & \multicolumn{2}{|c|}{ Consistence } & \multirow[b]{2}{*}{$\begin{array}{c}\mathrm{CO}_{3} \\
\text { stage }^{6}\end{array}$} & \multirow{2}{*}{$\begin{array}{l}\text { Effer- } \\
\text { ves- } \\
\text { cence } \\
(\mathrm{HCl})^{7}\end{array}$} & \multirow[b]{2}{*}{$\begin{array}{l}\text { Cemen- } \\
\text { tation }^{8}\end{array}$} & \multirow{2}{*}{$\begin{array}{c}\text { Hori- } \\
\text { zon } \\
\text { bound- } \\
\text { ary }^{9}\end{array}$} & \multirow[b]{2}{*}{ Roots $^{10}$} & \multirow[b]{2}{*}{ Pores ${ }^{11}$} & \multirow[b]{2}{*}{$\begin{array}{l}\text { Rhizo- } \\
\text { liths }^{12}\end{array}$} \\
\hline & & & & Wet & Dry & & & & Dry ${ }^{4}$ & Wet ${ }^{5}$ & & & & & & & \\
\hline & & & & & & & SCF & 2-T3 Profile & SP IV-C & ntinued & & & & & & & \\
\hline \multirow[t]{3}{*}{$\mathrm{S}_{\mathrm{F}}$} & $6 \mathrm{Kqmb2}$ & $175-200$ & F4 & $7.5 Y R 4 / 4$ & $\begin{array}{l}7.5 \mathrm{YR} \\
7 / 2-7 / 3\end{array}$ & $40-50$ & ls-s & $1 \mathrm{f}-\mathrm{m}$ sbk & h-vh & so po & III-IV & es & cs cont & $\mathrm{cs}$ & N.O. & N.O. & 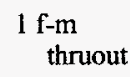 \\
\hline & 7Kqmb2 & $200-225$ & $\mathrm{~F} 3$ & $\begin{array}{l}7.5 \text { YR } 4 / 4 \\
6\end{array}$ & 7.5 YR $7 / 3-4$ & 70 & s & $1 \mathrm{f}-\mathrm{m}$ sbk & vh & so po & III & es & es cont & $\mathrm{gw}$ & N.O. & N.O. & $\begin{array}{l}2 \mathrm{f}-\mathrm{m} \\
\text { thruout }\end{array}$ \\
\hline & $8 \mathrm{~Kb} 2$ & $225-?$ & F3 & $10 Y R 5 / 3$ & $10 \mathrm{YR} 8 / 2-3$ & 70 & $\mathbf{s}$ & $1 \mathrm{f}-\mathrm{c} \mathrm{sbk}$ & $\mathrm{h}$ & so po & III & es & ci cont & not exp & N.O. & $1 \mathrm{f}-\mathrm{m}$ i clast & $1 \mathrm{f}-\mathrm{m} \mathrm{scat}$ \\
\hline
\end{tabular}

3.0 meters west from previous location dip on bedding gives same depth \#.

${ }^{1}$ From Munsell soil color charts, revised edition, 1990.

${ }^{2}$ Texture: $l=l$ loam, $s=$ sand, sit=silt.

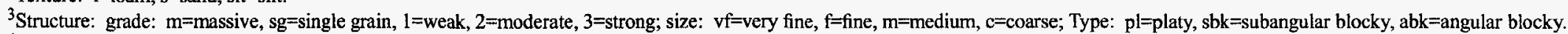

${ }^{4}$ Consistence, dry: lo=loose, so=soft, sh=slightly hard, $h=$ hard, vh=very hard, eh=extremely hard.

${ }^{5}$ Consistence, wet: $s 0=$ nonsticky, $s s=$ slightly sticky; $\mathrm{po}=$ nonplastic, $\mathrm{ps}=$ =slightly plastic.

${ }^{6}$ Carbonate morphology stage defined in Birkeland, 1984.

${ }^{7}$ Effervescence: in cold dilute hydrochloric acid, eo-noncalcareous, vse=very slightly effervescent, e=effervescent, em=moderately effervescent, es=strongly effervescent, ev=very strongly effervescent.

${ }^{8}$ Cementation: $\mathrm{CO}_{3}=$ carbonate, $\mathrm{SiO}_{2}=$ silicate; strength: $\mathrm{cw}=$ weak, $\mathrm{cs}=$ strong, $\mathrm{ci}=$ indurated; type: cont=continuous, discont=discontinuous

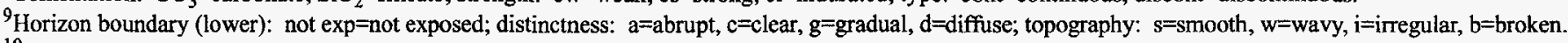

${ }^{10}$ Roots: abundance: $1=$ few, $2=$ common, $3=$ many; size: vf=very fine, $f=$ fine, $m=$ medium, $c 0=$ coarse; location: thruout=throughout, scatt=scattered, ped=ped faces, frac=fractures, lam=laminae.

"Pores: abundance: $1=\mathrm{few}, 2=\mathrm{common}, 3=$ many; size: vf=very fine, $\mathrm{f}=\mathrm{fine}, \mathrm{m=medium}$; shape: $v=$ vesicular, i=irregular or interstitial; location: thruout=throughout, scatt=scattered, ped=ped faces, nod $=\mathrm{CO}_{3}$, nodules, cis=clasts.

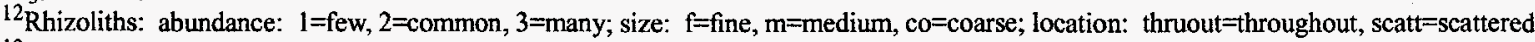

${ }^{13}$ Top of soil profile was disturbed during trench excavation. Horizon II starts at petrocalcic horizon not described in SP 1.

${ }^{14}$ Top of soil profile was disturbed during excavation. Ap horizon was not described. 
Samples for analysis have been collected from mapped lithologic units in trenches SCR-T1 and $\mathrm{SCR}-\mathrm{T} 3$ as part of an integrated program designed to provide direct geochronologic control for paleoseismic investigations at Yucca Mountain (Paces and others, 1994). This represents the first numerical dating efforts attempted on surficial deposits associated with the Stagecoach Road fault. The studies employed two basic dating techniques: (a) TL analysis of fine-grained, polymineralic sediment (table 4) and (b) U-series disequilibrium (U-Th) analyses of calcareous rhizoliths and matrix soil carbonate (tables 5,6). Samples in both trenches were collected primarily from uncemented sandy deposits on the hanging-wall blocks with the most complete paleoseismic record of late Quaternary faulting. Analyses of three samples for U-Th dating and six samples for TL dating have been completed from trench SCR-T1, and age estimates are available for one U-Th sample and five TL samples from trench SCR-T3. All sample locations are plotted in plates 2 and 3 except for one spurious TL sample (TL-17) and one U-Th sample (HD1439), which is located on the

Table 4. Age estimates based on thermoluminescence data

[Estimated dose, dose rate at both field and saturation moisture, and best age estimates based on the field soil-moisture. All uncertainties are quoted at the 95-percent confidence level ( 2 sigma) absolute; Gy, grays; ka, thousand years ago; SCR-T, Stagecoach Road trench; TL, thermoluminescence; \pm , plus or minus]

\begin{tabular}{ccccc}
\hline Trench & $\begin{array}{c}\text { Estimated } \\
\text { dose } \\
\text { (Gy) }\end{array}$ & \multicolumn{2}{c}{$\begin{array}{c}\text { Dose rate } \\
\text { (Gy/ka) }\end{array}$} & $\begin{array}{c}\text { Best age } \\
\text { estimate } \\
\text { (ka) }\end{array}$ \\
\cline { 3 - 4 } SCR-T1 & & Field & Saturation & \\
TL-02 & $95 \pm 46$ & $8.6 \pm 1.2$ & $8.2 \pm 1.2$ & $12 \pm 6$ \\
TL-26 & $75 \pm 3$ & $8.4 \pm 1.1$ & $7.5 \pm 1.0$ & $9 \pm 1$ \\
TL-25 & $103 \pm 4$ & $8.8 \pm 1.2$ & $7.9 \pm 1.1$ & $12 \pm 2$ \\
TL-16 & $210 \pm 42$ & $7.5 \pm 1.0$ & $6.0 \pm 0.9$ & $28 \pm 4$ \\
TL-27 & $392 \pm 67$ & $8.0 \pm 1.1$ & $7.2 \pm 1.0$ & $49 \pm 11$ \\
TL-15 & $26 \pm 0.3$ & $7.7 \pm 1.0$ & $6.2 \pm 0.9$ & $3 \pm 0.5$ \\
SCR-T3 & & & & \\
TL-28 & $20 \pm 2$ & $8.4 \pm 1.1$ & $7.2 \pm 1.0$ & $2 \pm 0.4$ \\
TL-18 & $185 \pm 31$ & $8.3 \pm 1.1$ & $6.6 \pm 0.9$ & $22 \pm 5$ \\
TL-19 & $691 \pm 110$ & $8.0 \pm 1.0$ & $6.4 \pm 0.9$ & $87 \pm 18$ \\
TL-29 & $476 \pm 124$ & $12 \pm 2$ & $9.3 \pm 1.3$ & $40 \pm 12$ \\
TL-17 & $318 \pm 17$ & $8.5 \pm 1.1$ & $6.8 \pm 0.9$ & $38 \pm 5$ \\
\hline
\end{tabular}

south wall opposite to the logged part of the trench. Analytical results are pending from additional $\mathrm{U}-\mathrm{Th}$ samples collected from both trenches in 1994. Thus, age determinations for units presented herein should be considered preliminary and possibly subject to change.

Correlations of at least one, and possibly two, basaltic ash-bearing layers present in both trenches with tephra deposits from nearby Lathrop Wells volcanic center at the south end of Yucca Mountain (Crowe and others, 1992, 1995) potentially provide independent age control. Four eruptive sequences have been identified at the volcanic center, and at least two, and possibly three, eruptions are believed capable of having introduced ash into deposits in the trench area. The two oldest eruptive events are associated with the most distinct tephra sheets near the vent and thus are most likely to have produced ashes exposed in trenches at Yucca Mountain. However, the thirdyoungest cone-building eruption also could have introduced ash locally at nearby sites such as the Stagecoach Road trenches. Considerable uncertainty in the ages of the volcanic units at the Lathrop Wells center presently limits their usefulness for providing direct age constraints. Preliminary ages of four tephra-producing sequences reported in Crowe and others (1995) are: (a) $\geq 100 \mathrm{ka} \mathrm{(100-130} \mathrm{ka)} \mathrm{for} \mathrm{the}$ oldest eruptive unit in the sequence; (b) 80 to $100 \mathrm{ka}$ for second-oldest units; (c) 40 to $60 \mathrm{ka}$ for third-oldest (cone-building) event; and (d) $<10 \mathrm{ka}$ for the small, youngest eruption. Preliminary results from dating in progress suggests that younger ages of 50 to $80 \mathrm{ka}$ for the second event and 30-60 ka for the third oldest event may be likely (Bruce Crowe, Los Alancos National Laboratory, written commun., 1995). A problem in using this ash chronology for independent age control is uncertainties in correlations of ashes in trench deposits with tephra from specific eruptive events. Provisional correlations are presented in this report primarily based on stratigraphic position and soil relationships in the trenches and at the vent area. Studies to determine the possibility of directly identifying ash-bearing units in the trenches on the basis of trace-element geochemistry are in progress. 
Table 5. Uranium and thorium isotopic data from carbonates collected from Stagecoach Road fault trenches SCR-T1 and SCR-T3

[Size fractions are given in mesh units. All isotopic ratios given as activity ratios. All uncertainties are relative (percentages) and are quoted at the 95 -percent confidence level ( 2 sigma); $U$, uranium; ppm, parts per million; Th, thorium; <, less than; \pm , plus or minus; dashes (-), no data available; $\rho$, correlation coefficient between isotope ratios; $\left.08,{ }^{230} \mathrm{Th} / 238 \mathrm{U} ; 28,{ }^{232} \mathrm{Th} /{ }^{238} \mathrm{U} ; 48,{ }^{234} \mathrm{U} /{ }^{238} \mathrm{U}\right]$

\begin{tabular}{|c|c|c|c|c|c|c|c|c|c|c|c|}
\hline Sample & $\begin{array}{c}\text { Size } \\
\text { fraction }\end{array}$ & $\begin{array}{c}\text { Analysis } \\
\text { type }\end{array}$ & $\underset{(\mathrm{ppm})}{\mathrm{U}}$ & $\begin{array}{c}\text { Th } \\
\text { (ppm) }\end{array}$ & ${ }^{234} U /{ }^{238} U$ & ${ }^{230} \mathrm{Th} /{ }^{238} \mathrm{U}$ & ${ }^{232} \mathrm{Th} /{ }^{238} \mathrm{Th}$ & ${ }^{230} \mathrm{Th} /{ }^{232} \mathrm{Th}$ & Pos-48 & $\rho_{28-48}$ & $\rho_{08-28}$ \\
\hline \multicolumn{12}{|c|}{ Rhizoliths from north wall, SCR-T1 } \\
\hline \multicolumn{12}{|l|}{ HD 1067} \\
\hline$-\mathrm{AL}$ & $<325$ & Leach & $9.77 \pm 1.8$ & $0.784 \pm 9.2$ & $1.5751 \pm 1.1$ & $0.3482 \pm 3.9$ & $0.0264 \pm 9.4$ & $13.17 \pm 9.2$ & 0.177 & 0.074 & 0.198 \\
\hline$-A W$ & $<325$ & Whole rock & $10.29 \pm 1.8$ & $0.973 \pm 5.9$ & $1.5530 \pm 1.1$ & $0.3310 \pm 3.2$ & $0.0312 \pm 6.2$ & $10.62 \pm 5.8$ & 0.206 & 0.107 & 0.267 \\
\hline$-B W$ & 325 to 200 & Whole rock & $11.80 \pm 1.9$ & $1.335 \pm 11.6$ & $1.5407 \pm 1.0$ & $0.3252 \pm 5.6$ & $0.0373 \pm 11.7$ & $8.702 \pm 11.5$ & 0.112 & 0.054 & 0.243 \\
\hline$-\mathrm{CW}$ & 200 to 100 & Whole rock & $10.99 \pm 1.8$ & $3.27 \pm 5.7$ & $1.5352 \pm 1.0$ & $0.3788 \pm 4.2$ & $0.0981 \pm 6.0$ & $3.862 \pm 5.5$ & 0.150 & 0.106 & 0.378 \\
\hline \multicolumn{12}{|c|}{ Rhizoliths from north wall, SCR-T1 } \\
\hline \multicolumn{12}{|l|}{ HD1068 } \\
\hline$-A L$ & $<325$ & Leach & $7.01 \pm 2.1$ & $0.614 \pm 5.5$ & $1.4895 \pm 1.1$ & $0.2524 \pm 3.3$ & $0.0289 \pm 5.9$ & $8.735 \pm 5.4$ & 0.202 & 0.115 & 0.286 \\
\hline$-A R$ & $<325$ & Residue & $8.24 \pm 5.1$ & $1.513 \pm 5.0$ & $1.4748 \pm 3.8$ & $0.2718 \pm 5.9$ & $0.0605 \pm 7.1$ & $4.492 \pm 5.1$ & 0.385 & 0.316 & 0.650 \\
\hline$-A W$ & $<325$ & Whole rock & $7.54 \pm 1.9$ & $0.926 \pm 5.6$ & $1.4783 \pm 1.1$ & $0.2448 \pm 3.6$ & $0.0405 \pm 6.0$ & $6.053 \pm 5.6$ & 0.186 & 0.111 & 0.288 \\
\hline$-B W$ & 325 to 200 & Whole rock & $6.93 \pm 1.7$ & $0.954 \pm 5.7$ & $1.4911 \pm 1.0$ & $0.2862 \pm 3.5$ & $0.0454 \pm 6.0$ & $6.311 \pm 5.7$ & 0.160 & 0.095 & 0.280 \\
\hline$-\mathrm{CW}$ & 200 to 100 & Whole rock & $8.05 \pm 2.5$ & $3.691 \pm 3.6$ & $1.4570 \pm 1.5$ & $0.2678 \pm 3.9$ & $0.1512 \pm 4.4$ & $1.771 \pm 3.5$ & 0.218 & 0.198 & 0.526 \\
\hline \multicolumn{12}{|c|}{ Individual rhizolith near main fault trace from south wall, SCR-T1 } \\
\hline \multicolumn{12}{|l|}{ HD1439 } \\
\hline A & - & Whole rock & $6.98 \pm 0.4$ & $0.73334 \pm 0.3$ & $1.5075 \pm 0.7$ & $0.1977 \pm 0.8$ & $0.0346 \pm 0.5$ & $5.706 \pm 0.8$ & 0.000 & 0.000 & 0.197 \\
\hline B & -- & Whole rock & $6.25 \pm 0.3$ & $2.61874 \pm 1.1$ & $1.4962 \pm 0.5$ & $0.2669 \pm 3.2$ & $0.1381 \pm 1.2$ & $1.933 \pm 3.4$ & 0.000 & 0.000 & 0.204 \\
\hline $\mathrm{C}$ & -- & Whole rock & $5.04 \pm 0.4$ & $0.60169 \pm 0.3$ & $1.4842 \pm 0.9$ & $0.2167 \pm 1.1$ & $0.0394 \pm 0.5$ & $5.507 \pm 1.0$ & 0.000 & 0.001 & 0.185 \\
\hline $\mathrm{D}$ & -- & Whole rock & $5.86 \pm 0.5$ & $4.22776 \pm 1.0$ & $1.4599 \pm 1.3$ & $0.3253 \pm 2.8$ & $0.2378 \pm 1.1$ & $1.368 \pm 3.0$ & 0.000 & 0.000 & 0.239 \\
\hline $\mathrm{C}-3$ & -- & Whole rock & $5.10 \pm 0.3$ & $0.61099 \pm 0.3$ & $1.4948 \pm 1.1$ & $0.2293 \pm 0.7$ & $0.0395 \pm 0.4$ & $5.806 \pm 0.7$ & 0.000 & 0.001 & 0.091 \\
\hline \multicolumn{12}{|c|}{ Matrix soil carbonate from north wall, SCR-T3 } \\
\hline \multicolumn{12}{|l|}{ HD 1447} \\
\hline$-A W$ & -- & Whole rock & $6.50 \pm 2.0$ & $2.747 \pm 5.0$ & $1.4758 \pm 1.5$ & $1.0010 \pm 3.4$ & $0.1392 \pm 5.4$ & $7.190 \pm 4.8$ & 0.272 & 0.171 & 0.359 \\
\hline$-B W$ & -- & Whole rock & $12.93 \pm 1.7$ & $2.924 \pm 6.1$ & $1.4859 \pm 0.8$ & $0.9762 \pm 3.9$ & $0.0746 \pm 6.4$ & $13.09 \pm 5.5$ & 0.122 & 0.074 & 0.436 \\
\hline$-\mathrm{CW}$ & -. & Whole rock & $4.64 \pm 2.1$ & $8.865 \pm 3.4$ & $1.3547 \pm 1.2$ & $0.9652 \pm 3.8$ & $0.6300 \pm 3.9$ & $1.532 \pm 2.5$ & 0.189 & 0.181 & 0.659 \\
\hline$-\mathrm{EW}$ & - & Whole rock & $5.54 \pm 1.9$ & $8.636 \pm 3.6$ & $1.3543 \pm 1.3$ & $0.9780 \pm 3.8$ & $0.5137 \pm 4.1$ & $1.904 \pm 3.0$ & 0.199 & 0.182 & 0.582 \\
\hline
\end{tabular}


Table 6. Uranium and thorium isotopic compositions of the authigenic carbonate component for sample aliquots listed in table 5

[Isotopic ratios are obtained by regressing ${ }^{234} \mathrm{U} /{ }^{238} \mathrm{U}-{ }^{230} \mathrm{Th} / 238 \mathrm{U}-232 \mathrm{Th} / 238 \mathrm{U}$ ratios simultaneously using maximum likelihood estirnation techniques (Ludwig and Titterington, written commun., 1994; Ludwig, 1993). All isotope ratios are given as activity ratios. All uncertainties are given at the 95-percent confidence level (2 sigma) absolute. The error correlation between ${ }^{234} \mathrm{U} /{ }^{238} \mathrm{U}-{ }^{230} \mathrm{Th} /{ }^{238} \mathrm{U}$ is listed under $\rho_{0848}$ A measure of the nonanalytical scatter is listed as the Mean Square of the Weighted Deviates (M.S.W.D.). Values greater than about two indicate that analytical errors cannot account for the observed scatter about the best-fit curve; ka, thousand years ago; \pm , plus or minus]

\begin{tabular}{ccccccc}
\hline Sample & $\begin{array}{c}\text { Number } \\
\text { of } \\
\text { aliquots }\end{array}$ & ${ }^{234} \mathbf{U} /{ }^{238} \mathbf{U}$ & ${ }^{230} \mathrm{Th} /{ }^{238} \mathbf{U}$ & $\mathbf{P}_{\mathbf{0 8}-48}$ & M.S.W.D. & $\begin{array}{c}\text { Age } \\
\text { (ka) }\end{array}$ \\
\hline HD1067 & 4 & $1.5673 \pm 0.042$ & $0.3171 \pm 0.0325$ & 0.169 & 3.36 & $24 \pm 3$ \\
HD1068 & 5 & $1.4963 \pm 0.0466$ & $0.2559 \pm 0.0246$ & 0.207 & 7.27 & $20 \pm 2$ \\
HD1447 & 4 & $1.5055 \pm 0.0248$ & $0.9942 \pm 0.0612$ & 0.203 & 2.00 & $108 \pm 10$ \\
HD1439 & 5 & $1.5050 \pm 0.116$ & $0.1901 \pm 0.0247$ & 0.001 & 110 & $15 \pm 2$ \\
\hline
\end{tabular}

\section{STRATIGRAPHY, SOILS, AND AGES OF LITHOLOGIC UNITS IN TRENCHES AND BOREHOLES}

\section{General Characteristics of Trench Stratigraphy}

The Stagecoach Road fault in trenches SCR-T1 and SCR-T3 juxtaposes markedly different stratigraphic sequences of mixed alluvium, colluvium, and eolian material on the footwall and hanging-wall blocks (figs. 7, 8; pls. 1-3). The footwall blocks consist of a 2- to 3-m thickness of sand and gravel containing thick, well-developed petrocalcic soils. These units are deposited on a gently west-sloping erosion surface cut across upper Tertiary volcanic bedrock. The hanging-wall blocks comprise a much younger depositional sequence of unconsolidated to poorly consolidated silty to pebbly sand with weakly to moderately developed soils. One or more thin, undeformed layers of sand and silt continue across the entire length of the trench and shallowly bury the uppermost trace of the fault zone in both trenches.

\section{Lithologic Units}

The logs portray a detailed trench stratigraphy defined for each site on the basis of lithologic field descriptions (figs. 7, 8; pls. 2, 3; table 2; appendixes A, B). The alphanumeric designations of mapped units reflect the following stratigraphic hierarchy. At each site, major lithologic sequences (uppercase letters), ranging from 0.5 to $3 \mathrm{~m}$ in thickness, are identified on the basis of variations in texture, color, degree of cementation, sedimentary features, and associated soil-profile development. Most of these sequences consist of several depositional units (designated by numbers) with typical thicknesses of 0.2 to $2 \mathrm{~m}$ that are defined on the basis of relatively less distinct lithologic variations, layer geometry, and bedding contacts. Where necessary, some of the numbered depositional units are subdivided into two to five subunits (designated by lowercase letters), which are commonly $\leq 1 \mathrm{~m}$ thick. These subunits could represent internal facies (fissure and debris facies within a colluvial wedge; Nelson, 1992), distinct localized facies within a larger unit (small gravelly channels within a general sandy layer), or small bedding packages within a thick, lithologically homogeneous unit.

Lithologic sequences and units in the footwall blocks differ significantly between the trenches, largely reflecting the differing geomorphic characteristics of the upslope piedmont at each site (fig. 3). In contrast, there is general similarity between stratigraphic units in the hanging-wall deposits, particularly the younger units in the upper part of the trenches. There is greater proportion of gravelly alluvium in trench SCR-T3, relative to the predominantly sandy deposits in trench SCR-T1. Table 7 shows provisional lithostratigraphic correlations of the major hangingwall sequences in each trench, derived primarily from observed similarities in lithology, soil develojment, and relative stratigraphic position.

The type and origin of lithologic units in the trenches, although variable, is dominated by combinations of alluvial, colluvial, and eolian surficial 


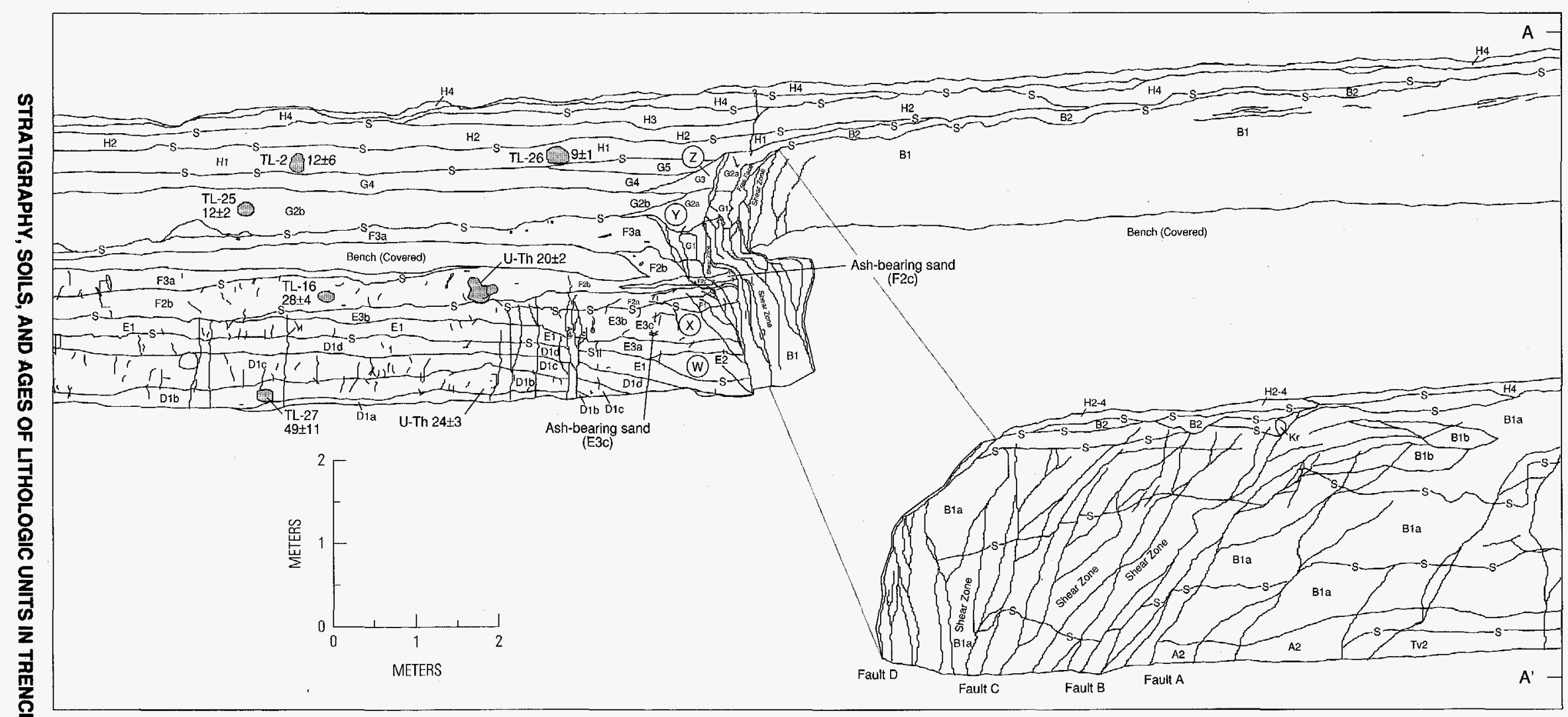

Figure 7. Portion of log, looking north, of Stagecoach Road fault in trench SCR-T1 (modified from plate 2).

응 


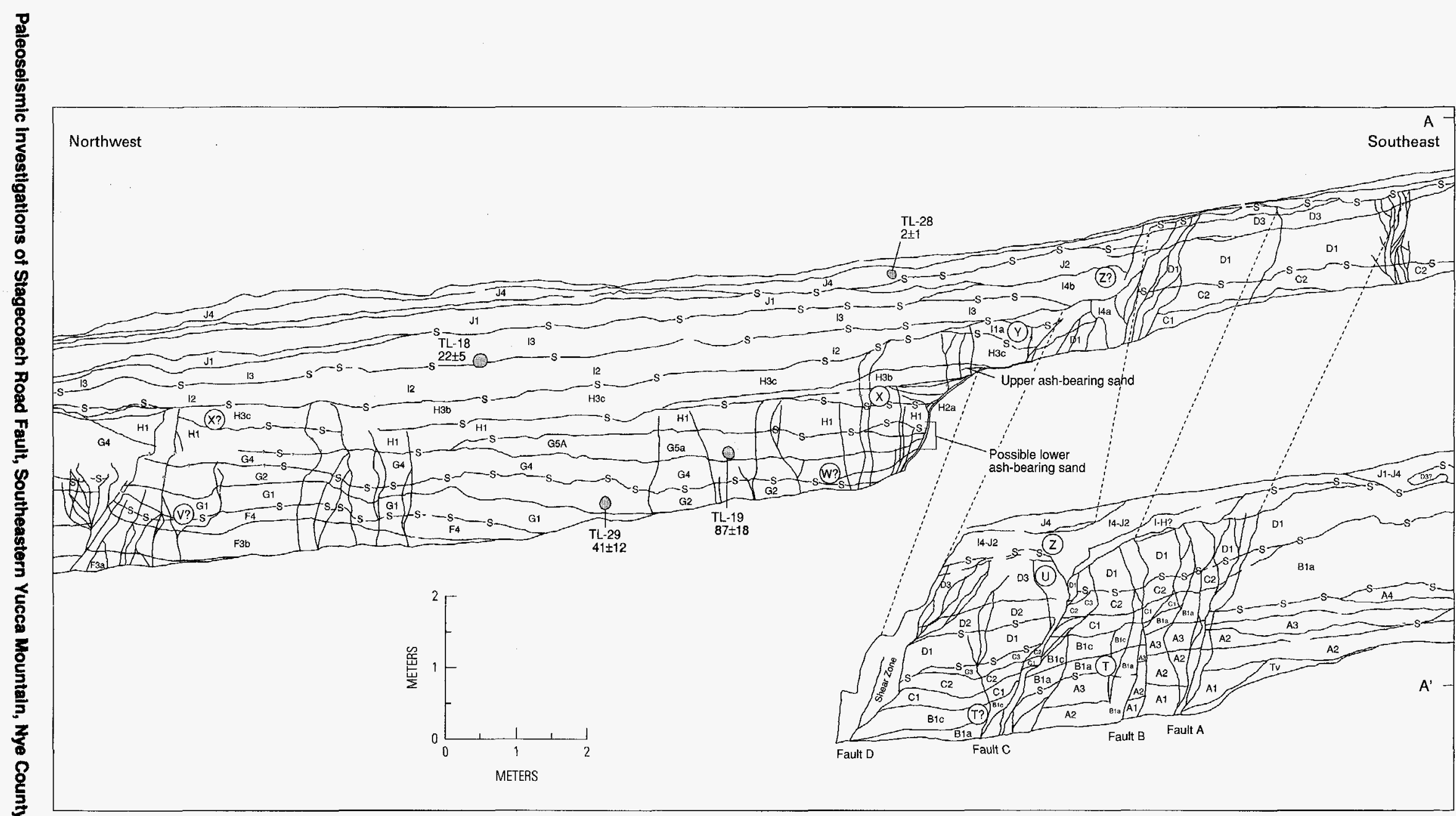

Figure 8. Portion of log, looking north, of Stagecoach Road fault in trench SCR-T3 (modified from plate 3). 
Table 7. Lithostratigraphic correlations and estimated ages of lithologic sequences in trenches SCR-T1 and SCR-T3

[General ages assigned based on soil stratigraphy and numerical age ranges derived from geochronologic control in tables 4 and 6 , queried where uncertain. Sequences are described in text and are shown in plates 2 and 3 ; component units are described in table 2 and appendixes $A$ and $B$. Basaltic ash also indicated. Age symbols are $\mathrm{H}$, Holocene; IsP, latest Pleistocene; IP, late Pleistocene; mP, middle Pleistocene; eP, early Pleistocene; Pl, Pliocene; $1 \mathrm{M}$, late Miocene; $\mathrm{mM}$, middle Miocene; $\mathrm{ka}$, thousand years ago; Ma, Million years ago; dashes (--), no data; $>$, greater than]

\begin{tabular}{|c|c|c|}
\hline SCR-T1 & SCR-T3 & $\begin{array}{c}\text { Estimated ages } \\
\text { (ka) }\end{array}$ \\
\hline \multicolumn{3}{|c|}{ Lithostratigraphic correlations-hanging-wall sequences } \\
\hline $\mathrm{H}$ & $\mathrm{J}$ & H-lsP $(0-15)$ \\
\hline $\mathrm{G}$ & I & lsP $(10-30)$ \\
\hline $\mathrm{F}(\mathrm{ash})$ & $H$ (upper)(ash) & IsP (25-40?) \\
\hline $\mathrm{E}$ (ash?) & $\mathrm{H}$ (lower) & IsP $(40 ?-50)$ \\
\hline $\mathrm{D}$ & G (upper) & $1 P(50-100 ?)$ \\
\hline-- & $\mathrm{G}$ (lower) & IP $(70-120)$ \\
\hline- & $\mathbf{F}$ & $1-m P(>110)$ \\
\hline \multicolumn{3}{|c|}{ Lithostratigraphic correlations-footwall sequences } \\
\hline $\mathrm{H}$ & $\mathrm{I}-\mathrm{J}$ & $\mathrm{H}-1 \mathrm{sP}$ \\
\hline $\mathrm{C}$ & $\mathrm{E}$ & $1-\mathrm{mP}$ \\
\hline- & D & $\mathrm{mP} ?$ \\
\hline-- & $\mathrm{C}$ & $\mathrm{mP} ?$ \\
\hline- & B & $\mathrm{mP} ?$ \\
\hline-- & A & $\mathrm{mP} ?$ \\
\hline B & -- & $\mathrm{m}-\mathrm{eP}$ \\
\hline A & -- & $\mathrm{m}-\mathrm{eP}$ \\
\hline Tv2 & -- & 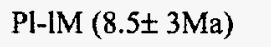 \\
\hline Tv1 & Tv & $\mathrm{mM}$ \\
\hline
\end{tabular}

deposits. The alluvial deposits typically consist of intermixed, poorly sorted pebble-cobble gravels and sand. Bedding is usually poorly defined, although some gravel lenses display lenticular channel geometries. Colluvial deposits generally comprise mixtures of sand, silt, and pebbly gravels reworked to varying degrees by local rilling and slope wash. One or more thin, loess-like, eolian mantles of fine sand and silt lie just below the surface in both trenches. Large sections of the trenches, particularly in the hanging-wall blocks, contain thick layers of poorly bedded to massive pebbly sand and silt, predominantly of eolian origin, with varying degrees of local reworking by surface runoff. Numerous rhizoliths (carbonate-replaced fossilized roots) and root casts are distributed throughout many of these sand units.
Small, commonly indistinct, colluvial units of silt, sand, and gravel occur in the hanging wall adjacent to the fault zone. Some of these features are interpreted as colluvial wedges related to the degradation of fault scarps produced by surface ruptures along the fault, as outlined by Wallace (1977), Swan and others (1980), and Nelson (1992). These scarpderived colluvial wedges commonly overlie debrisfilled fissures that developed during ground rupture along the fault zone. Many of these wedges occur in sandy intervals near the fault zone and thus are difficult to identify due to absence of a clastic debris facies, indistinct internal stratification and bounding contacts, and overprinting by subsequent deformation and rhizolith development.

\section{Soil Stratigraphy}

Soils were described along three to four vertical profiles (designated by Roman numerals, such as SP II) located on the footwall and hanging-wall blocks in each trench (pls. 2, 3; table 3). Each soil profile location spans the full vertical extent of the trench wall at that location and thus commonly encompasses the surface soil and several buried soils. Individual soil units are identified according to the lithologic sequence in which they are developed at that location $\left(\mathrm{S}_{\mathrm{H}-\mathrm{J}}\right)$.

The stratigraphic units in both trenches contain surface soils and buried paleosols that both show variable weak to strong degrees of development. The strongest soil development generally occurs in the upper part of the footwall blocks, which exhibit thick, well-cemented petrocalcic soils with carbonate morphologies of Stage III-IV, locally approaching Stage V in trench SCR-T1, using the definitions of Birkeland (1984) and Machette (1985). These thick, near-surface relict soils are mantled by generally thin colluvial deposits with weakly developed soils characterized by Stage I carbonate and (or) silica cementation (SP I and II, pls. 2, 3). The uppermost colluvial units commonly fill shallow pits or depressions in the upper part of the subjacent petrocalcic soils. The origin of these features is unclear but probably includes bioturbation and (or) degradation (scour, dissolution) of the top of the carbonate soil.

Soils in the hanging-wall deposits are weakly developed compared to soils in the footwall blocks. This contrast is particularly obvious in units adjacent 
to the fault zone. Locally strong soils with Stage IIIIV carbonate-silica cementation occur in the lower units at the western (hanging wall) ends of trenches (for example, SP IV, pl. 3) west of any significant secondary deformation along the fault zone. Hangingwall deposits in the lower one-half to two-thirds of the trenches near the fault contain weakly to moderately developed soils with Stage I-III carbonate morphologies. Most of the visible carbonate is concentrated in rhizoliths and root casts. Only very weakly developed soils with maximum Stage I carbonate morphology are present in the upper section of hanging-wall deposits. Soils in the hanging wall also notably lack deep rubification or textural evidence for clay accumulation associated with argillic horizon development. This weak soil development probably reflects the youthfulness of the deposits; however, it may be influenced texturally by the predominance of eolian sand and silt in the parent material. The sandy textures may induce widespread dissemination of carbonate and silica, which may in part explain the weak morphologic stages in the visible accumulation of these constituents. Soil horizons commonly are poorly defined in the hanging wall owing to gradational boundaries, which suggest cumulic soil development in an eoliandominated aggradational environment.

\section{Trench SCR-T1}

Two bedrock units are exposed at the base of the trench in the footwall block (pl. 2). A cemented tuffaceous sandstone (Tv1) probably associated with the middle Miocene Paintbrush or Timber Mountain Groups is present at the eastern end of the trench. This unit is juxtaposed across the eastern strand of the Stagecoach Road fault against a poorly indurated, pumiceous tephra deposit of unknown stratigraphic affinity or age (Tv2). This latter rock type is not clearly associated with the main upper Miocene volcanic sequence, but rather may be related to latest Miocene or Pliocene volcanism (D.C. Buesch, U.S. Geological Survey, written commun., 1993). A sample of the Tv2 unit has been submitted for $\mathrm{K}-\mathrm{Ar}$ dating. A preliminary age for this unit is $8.5 \pm 3 \mathrm{my}$ (Edward McKee, U.S. Geological Survey, written commun., 1994).

A weathering zone developed on bedrock (Sequence A) is overlain by a 2- to 3-m-thick package of mixed alluvial, colluvial, and eolian deposits
(Sequence B). A thick, very well cemented petrocalcic soil with Stage III-V carbonate morphologies has developed though the entire sequence and obscures the internal stratigraphy of the parent material. Unit $C$ is colluvial fill within pits in the upper surface of the petrocalcic horizon.

A significant time-stratigraphic gap occurs between footwall units and the lowest deposits on the hanging-wall block. Neither bedrock nor deposits with well-developed soils comparable to the petrocalcic soil on the footwall block are exposed in the hanging-wall portion of the trench. The two boreholes drilled on the hanging wall west of the fault reveal 20 to $30 \mathrm{~m}$ of sandy deposits above a 30 - to 50-m-thick section of clay-rich sand with local interbeds of alluvial gravel. The hanging-wall sequence contains only a few weakly to moderately developed calcic soils with Stage I to III morphologies that are locally associated with clay-rich $\mathrm{Bt}$ soil horizons. Stage I-type carbonate is also dispersed throughout much of the upper sandy section with little or no concentrations into definable soil horizons. No thick, well-developed petrocalcic soils were encountered in the depositional sequence above bedrock in either borehole. Bedrock was penetrated at a depth of $83 \mathrm{~m}$ in the SR-2 borehole, and depth to bedrock increases to $104 \mathrm{~m}$ to the west in borehole SR-3.

Within trench SCR-T1, two basal sandy units containing abundant carbonate-cemented rhizoliths and root casts occupy the lower part of the hanging wall (Sequences D and E; fig. 7; pl. 2). These sequences are overlain by a poorly exposed sand unit (Sequence F) with a weak, but distinct, carbonate soil and scattered rhizoliths. At least one, and possibly two, thin sand layers deposited in Sequences $E$ and $F$ adjacent to the fault contain dispersed (5-15 percent) basaltic ash.

The stratigraphic sequence in the upper section of the trench consists of loose, poorly stratified sand and silt layers with minimal soil development. Sequence $\mathrm{G}$ is moderately oxidized in contrast to the minimally weathered overlying deposits of Sequence $\mathrm{H}$. The latter sequence buries the fault trace, but thins eastward across the upper surface of the petrocalcic soil capping the footwall block (fig. 7; pl. 2). Sequence $H$ is the only deposit in the trench present on both sides of the fault; all of the underlying hanging-wall deposits (Sequence $D$ through $G$ ) are truncated against the Stagecoach Road fault. 


\section{Trench SCR-T3}

On the footwall block, a small exposure of bedrock (a welded tuff unit of middle Miocene Paintbrush Group) occurs near the trench base adjacent to the main fault zone (Tv; fig. 8; pl. 3). Above this bedrock unit are a series of mixed alluvial, colluvial, and eolian units comprising the bulk of surficial deposits in the footwall block. The basal sequence consists of gravelly alluvium with several distinct channel lenses (Sequence A). These coarse deposits are overlain by a thick sequence of rhizolithrich sand with minor gravel layers that at the west end of the footwall drapes across and is cut by the eastern strand of the fault zone (Sequences B and C; fig. 8; pl. 3). Another gravelly alluvial deposit (Sequence D) mantled by thin, fine-grained colluvial and eolian material caps the footwall sequence. Two distinct, well-developed soils with Stage III-IV carbonatesilica cementation are present at the top of

Sequences A and D. The upper surface of the upper petrocalcic soil contains pits filled with colluvium (Unit C).

The hanging-wall block contains a basal sequence of alluvial gravels, moderately to well cemented with Stage III-IV carbonate (Sequence F; fig. 8; pl. 3), that are exposed only at the west end of the trench. Overlying these gravels are a series of sand layers (Sequences $\mathrm{G}$ and $\mathrm{H}$ ) which collectively thicken eastward until truncated by the westernmost strand of the main fault. These sandy deposits contain numerous rhizoliths and several paleosols discontinuously cemented with carbonate Stage I-II morphologies. A thin sand layer (Unit $\mathrm{H} 3 \mathrm{a}$ ) with very sparse basaltic ash ( $<5$ percent) occurs at bench level in Sequence $H$, in a similar relative stratigraphic position to the upper ash-bearing sand in trench SCR-T1.

The upper part of the hanging-wall sequence in trench SCR-T3 consists of poorly consolidated to unconsolidated layers of fine sand and silt with little or no soil development. The lower, slightly reddened layers (Sequence I) abut against one of the western strands of the Stagecoach Road fault. The thin, eoliandominated units in Sequence J are deposited against and in part overlap across the main fault trace without displacement; a lithologically similar sequence discontinuously mantles the main stratigraphic sequence and related soil on the footwall block.

\section{Boreholes SR-1, SR-2, and SR-3}

Boreholes SR-1, SR-2, and SR-3 penetrated a 83-m to 104-m thickness of unconsolidated to poorly consolidated sediments above weathered bedrock in the hanging-wall block. The boreholes are located 49 to $76 \mathrm{~m}$ west of the Stagecoach Road fault in Trench SCR-T1. Most of the samples in these boreholes consist of uncemented to weakly cemented, poorly bedded to massive silty sand layers interstratified with a few thin scattered sandy gravel layers. The section contains variable amounts of dispersed calcium carbonate but lacks thick, very well developed petrocalcic or calcic soils similar to the petrocalcic soil with carbonate morphologies of Stage III-V in the footwall block of the trench. Most of the carbonate in the core samples consists of Stage I or II nodules or carbonate coatings on clasts intermixed with Stage IIIII carbonate chips that are locally concentrated into zones suggesting moderately developed petrocalcic soil horizons. For example, several thin carbonate Stage III-IV(?) petrocalcic soils are interpreted at depths of $22,36,61$, and $72 \mathrm{~m}$ in boreholes SR-1 and SR-2.

In Borehole SR-2, a basal layer of alluvial gravel directly overlies a welded tuff unit of the Ranier Mesa Tuff at a depth of $83 \mathrm{~m}$. A similar welded tuff was penetrated at a depth of $104 \mathrm{~m}$ in Borehole SR-3. The welded tuff is overlain by a 4.3 -m-thick zone of highly weathered reworked pumice-rich tuff that provisionally is correlated with Unit Tv2 in the footwall block of Trench SCR-T1. This establishes a vertical displacement of $100 \mathrm{~m}$ on this unit, which represents a minimum value because Unit Tv2 is present on the western (downthrown) side of the easternmost fault strand in the footwall block (pl. 2). The primary fault zone in the center of the trench is not intersected in any of the boreholes. The contact between bedrock and overlying sediments is depositional in nature, and is interpreted as a downfaulted equivalent of the bedrock pediment present in and to the east of Trench SCR-T1.

\section{Estimated Ages of Stratigraphic Sequences}

General ages of the lithologic sequences in the trenches are estimated by correlations with the regional Quaternary allostratigraphy of the Yucca 
Mountain area (see table 7, Wesling and others, 1992; John Wesling, written commun., 1993; Lundstrom and others, 1993, 1994), using criteria such as lithology, stratigraphic position, and degree of pedogenesis. The very thick, well-cemented petrocalcic soil capping the footwall of trench SCR-T1 is at least as strongly developed as relict surface soils on Q1 deposits of middle to early Pleistocene age; the associated deposit (B1) may be as old as Pliocene. The strongly developed petrocalcic soils in the footwall units of trench SCR-T3 (Sequence A to D) suggest correlations with $\mathrm{Q} 1$ to $\mathrm{Q} 3$ deposits of middle Pleistocene age. In contrast, the weak to moderate degree of soil development in hanging-wall units of both trenches indicates generally young, late Pleistocene to Holocene ages associated with Q4 and Q5 deposits. The carbonate-cemented alluvial gravels in the lower western section of trench SCR-T3 may be slightly older, perhaps correlative to $\mathrm{Q} 3$ deposits of late middle or early late Pleistocene age. These assignments also are consistent generally with specific age estimates provided by the geochronologic (U-Th and TL) dating of deposits and soil features in both trenches (table 7; see "Geochronology" section). Provisional stratigraphic correlations with basaltic tephra units at the Lathrop Wells volcanic center are difficult to utilize effectively given uncertainties arising from correlations with specific source tephra and age determinations of eruptive events, although late Pleistocene age for sediments containing ash is consistent with the provisional age assessments for Lathrop Wells events cited earlier.

\section{Geochronology}

\section{General Methods and Results}

Results from the TL studies are given in table 4. Analytical techniques follow the procedures of H.T. Millard and P.B. Maat (U.S. Geological Survey, written commun., 1994), and Paces and others (1994). A fundamental assumption of TL dating is the rezeroing of electron traps by ionizing radiation (sunlight) at the time of sediment transport. If met, the ages reported in table 4 should provide estimates of the time since the sediment was last exposed to light and buried. This assumption appears to be satisfied for most of the fine-grained deposits at Yucca Mountain (Paces and others, 1994); however, one sample of a near-surface unit in trench SCR--T3 yields an anomalously old age indicative of incomplete zeroing (sample TL-17). Ages for lithologic units on the hanging-wall block range from a likely minimum of approximately $2 \mathrm{ka}$ to approximately $100 \mathrm{ka}$ with 95-percent $(2 \sigma)$ confidence-level uncertainties typically about 20 to 25 percent of the quoted age. Estimated doses (ED) listed in table 4 are determined by the Total Bleach technique, although for most samples, ED's also are derived from the Partial Bleach technique. When available, the two ED values typically agree within $1 \sigma$ error overlap. An additional source of uncertainty in TL age estimation is the history of soil-moisture contents. Pore water attenuates the in-situ radiation stimulating the TL response. Therefore, longer exposure times are necessary for wet sediments to achieve the same cumulative radiation dose relative to dry sediments. Present field soilmoisture contents are low for sandy soils typically encountered in the hanging-wall blocks of the Stagecoach Road trenches (several percent by weight), but moisture contents may have been significantly higher during past pluvial cycles. Table 4 gives ages standardized to field moisture contents which thus represent minimum age estimates.

Another potential problem for TL analyses is the post-depositional translocation of silt and very fine sand into these units. It seems possible to mechanically introduce a fine sediment fraction significantly after deposition of this unconsolidated and permeable sandy parent material. Such a mechanism might be difficult to detect analytically and would tend to produce anomalously young TL ages for that unit such as TL-15 in trench SCR-T1.

Uranium and thorium isotopic data for: pedogenic carbonates used to calculate U-series (U-Th) disequilibrium ages are given in table 5. Many of the lithologic units in both trenches contain well-preserved and locally dense carbonate-impregnated rhizoliths. Although these fossilized roots lend themselves well to U-Th dating techniques, the timing of plant growth relative to sediment deposition remains a vexing question. Rhizoliths clearly represent a minimum age for the deposit itself because there is an unknown, possibly long, interval between the time of deposition and root introduction. In addition, several discontinuous carbonate soils of varying degrees of development were sampled for dating. Pedogenic carbonate in these soil horizons 
also represent minimum soil ages postdating sediment deposition by some finite accumulation time.

The principles of U-series disequilibrium dating, as well as the analytical technique used for carbonates, are described elsewhere (Szabo and others, 1981; Paces and others, 1994). The relatively low values of ${ }^{230} \mathrm{Th} /{ }^{232} \mathrm{Th}$ indicate that all of the fractions listed in table 5 require corrections for addition of detrital ${ }^{230} \mathrm{Th}$. In order to accomplish this, samples were prepared either by mechanical fractionation of softer, authigenic carbonate and the harder detrital components or by leaching of carbonate with dilute nitric acid. Isotopic analyses were conducted either by counting alpha particle disintegrations by an alpha spectrometer or by ion counting in a thermalionization mass spectrometer. The results of both methods provide a sufficient spread of ${ }^{232} \mathrm{Th} /{ }^{238} U$ to allow regression back to a ${ }^{232} \mathrm{Th}$-free component. The isotopic compositions of this carbonate component are assumed to reflect an episode of enhanced soil formation that subsequently behaved as a closed system with respect to evolution of ${ }^{238} \mathrm{U},{ }^{234} \mathrm{U}$, and ${ }^{230} \mathrm{Th}$. The results of regression on these three components, using maximum-likelihood estimation techniques (table 6), are used to derive age estimates ranging from 15 to $24 \mathrm{ka}$ for rhizoliths and $108 \mathrm{ka}$ for matrix soil carbonate, using the techniques of Ludwig (1993), and K.R. Ludwig and D.M. Titterington (U.S. Geological Survey, written commun., 1994). Uncertainties are quoted at the 95 -percent $(2 \sigma)$ confidence level and range between 10 and 15 percent of the cited age.

\section{Geochronologic Control in Trench SCR-T1}

The geochronologic control for deposits in the lower hanging-wall block at and below the mid-level bench are summarized as follows. A TL sample collected from unit F2b straddling the bench has yielded an age of $28 \pm 4$ (TL-16), and a TL age of $49 \pm 11$ (TL - 27) is available from unit D1b near the base of the trench (fig. 7; table 4). The latter older age creates severe mismatch problems in correlations between the two trenches of lithostratigraphic units (table 7) and faulting events (see later section on age constraints on faulting); thus, the age may be too young due to post-depositional contamination by silt and fine sand. These TL results indicate that the U-Th ages of $20 \pm 2$ and $24 \pm 3$ (samples HD1068 and HD1067, respectively, table 6) from rhizoliths collected in Sequences F and D represent minimum ages related to post-depositional root invasion. This inference is supported by the presence of a strongly developed buried calcic soil in Sequence $E$ between the two root zones. Collectively, these estimates indicate that the age of deposits in the lower hanging wall below the bench are at least 40 to $60 \mathrm{ka}$, and may be significantly older. The deposits and carbonate soil straddling the bench are generally 25 to $40 \mathrm{ka}$ in age (fig. 7; table 7).

However, these age assignments based primarily on TL ages do present a potential problem with the presence of dispersed basaltic ash in units F2c and possibly E2c within this stratigraphic interval.

These ash-bearing layers were initially correlated with the second oldest and oldest tephra units, respectively, from the Lathrop Wells cone (Crowe and others, 1995). The $50-80 \mathrm{ka}$ and $100 \mathrm{ka}$ ages of these eruptions (Bruce Crowe, Los Alamos National Laboratory, oral commun., 1995) are significantly older than the TL ages from interstratified units cited above. This discrepancy can be minimized if the two ash-bearing units are correlated with third- and second-oldest tephra units in the vent area. Traceelement geochemical data from ash in the upper layer in the trench supports, but does not require, the original correlation with the second-oldest eruption (Frank Perry, Los Alamos National Laboratory, oral commun., 1995). Alternatively, this correlation may be correct and the TL ages are anomalously young, perhaps as a result of post-depositional translocation of silt as noted previously. This apparent contradiction has not yet been resolved, although the preferred interpretation favors acceptance of most of the $T L$ ages and using the second ash correlation scheme described above.

Unit $\mathrm{H} 1$ in the upper section of the hanging wall contains two TL dates of $12 \pm 6 \mathrm{ka}$ and $9 \pm 1 \mathrm{ka}$ (samples TL-02 and TL-26, respectively), which are generally concordant with one another within analytical uncertainty. These ages appear stratigraphically reasonable in that they are consistent with a weak carbonate soil at the top of Unit $\mathrm{H}$ that resembles soils observed in many lower Holocene to uppermost Pleistocene Q5 deposits in the Yucca Mountain region (Wesling and others, 1992; John Wesling, Geomatrix, Inc., written commun., 1993; Lundstrom and others, 1993, 1994). TL dates of $3 \pm 0.5 \mathrm{ka}$ and $12 \pm 2 \mathrm{ka}$ (samples $\mathrm{TL}-15$ and $\mathrm{TL}-25$, respectively) are available from the base of sequence $G$ immediately 
above the bench in the upper section of the hanging wall (fig. 7, table 4; Paces and others, 1994). The younger of these two dates appears anomalously young because this age is out of stratigraphic sequence with the overlying unit, and the age is inconsistent with both the degree of weathering of the deposit itself, as well as the amount of pedogenesis in the overlying Sequence $H$. Thus, the older age is preferred for this unit. This age plus lithostratigraphic and pedogenic correlations with a dated deposit in Sequence I at base of upper wall in trench SCR-T3 (see below) indicate an general age range of latest Pleistocene (10-30 ka) for Sequence $G$ in this trench (see figs. 7, 8; tables 4, 7). Within error bounds, the combined age control indicates a probable Holocene to latest Pleistocene age ( $\leq 30 \mathrm{ka}$ ) for Sequences $\mathrm{H}$ and $\mathrm{G}$ in the upper part of the hanging wall of this trench.

A rhizolith collected from within the main fault zone near the base of the unlogged portion of the south wall of the trench yielded a U-series age of $15 \pm 2 \mathrm{ka}$ (table 6). The exact relationship between this sample and faulting is not clear, although the rhizolith was not obviously deformed and may reflect deep root penetration along the fault following the most recent event.

\section{Geochronologic Control in Trench SCR-T3}

In the lower section of the hanging-wall block, a TL sample from Sequence G (TL-19, Unit G5) has yielded an age of $87 \pm 18 \mathrm{ka}$ (sample TL-19; fig. 8; table 4). This age directly conflicts with a TL age of $40 \pm 12$ ka from a sample (TL-29) in unit G2 lower in the section. It is difficult to resolve this problem. Two different alternative age assignments exist. In the first alternative (Alternative A), the older age is accepted and the younger age is rejected as being too young, perhaps due to post-depositional contamination by translocated silt and fine sand. This alternative is preferred because it is in good agreement with a U-Th date of $108 \pm 10 \mathrm{ka}$ that was derived from carbonate laminae at the base of the strong soil with carbonate Stage II-IV morphology developed on Units G4 and G2 west of the western graben-bounding fault (sample HD1447; fig. 8; SP IV, pl. 3; table 6). The units containing this soil continue eastward across the graben and underlie Unit G5 with the TL date. No comparably strong carbonate soil is present in the sandy units in this part of the graben; however, a weakly to moderately developed, buried calcic soil in Unit H1 extends downward through Units G5 to G1 and may correlate with at least part of the more strongly developed soil in Unit G4 west of the graben (Profiles SP III and IV, pl. 3). Also, the older age assignment is in better agreement with the $50-80 \mathrm{ka}$ age of the ash using the most straightforward correlation with the second oldest tephra at the Lathrop Wells source. These data indicate a broad age range of 70 to $120 \mathrm{ka}$ for at least Sequence $\mathrm{G}$ in the lower vall of the trench. The underlying alluvial gravels of Sequence $F$ contain a moderately cemented, buried petrocalcic soil which indicates a possible, but poorly constrained, age of $\geq 110-150 \mathrm{ka}$ for units at the base of hanging-wall exposures in the trench.

As a second alternative (Alternative $\mathrm{B}$ ), the younger TL age of $40 \pm 12 \mathrm{ka}$ (TL-29) could be correct, which would place the older TL age (TL-19) in error and call into question either the U-series age itself (HD-1447) or its correlation described above into the graben sequence. The age of sample TL-29 lies within the error of the age of $49 \pm 11 \mathrm{ka}$ (TL-27) from Unit D1 in trench SCR-T1 (see previous section). Thus, it seems plausible that both units correlate with one another and a composite age of $45 \pm 8 \mathrm{ka}$ is computed for the single correlated horizon from the weighted average of the two age determinations, where the weights are the inverse squares of the corresponding standard deviations (Taylor, 1982). This establishes a general age range for sequence $G$ of $30-60 \mathrm{ka}$ in this alternative age assignment.

A TL sample from Sequence I in the middle section of the hanging-wall block, above the bench, yields an age of $22 \pm 5 \mathrm{ka}$ (sample TL-18; fig. 8; table 4). This age appears consistent with the degree of soil development that includes slight oxidation and local ped formation. The underlying Sequence $\mathrm{H}$ contains a weakly to moderately developed calcic soil and a sand layer with sparse basaltic ash sand, similar to units at mid-bench level in trench SCR-TI described earlier. We assign a provisional age of 20-50 ka for Sequences I and H in trench SCR-T3 (table 7) based both on specific geochronologic control in the trench and correlation with Sequences $G$ through E in trench SCR-T1.

The age control is poor for the upper section of hanging-wall block in this trench. A TL sample (TL-17) collected from the uppermost eolian unit (J1) yielded an age of $38 \pm 5 \mathrm{ka}$ (table 4 ), which appears anomalously old for a surficial deposit with no significant soil development. This suggests that the TL signal in the deposit may not have reset completely 
during deposition. It is also possible that the material from the nearby spoil pile from the trench may have contaminated the sample during collection. Another $T L$ sample collected from the same location yielded an age of $2 \pm 0.4 \mathrm{ka}$ (TL-28; fig. 8; table 4). Sequence J is considered Holocene to latest Pleistocene in age $(\leq 20 \mathrm{ka})$, based on the resampled TL date, the minimal degree of pedogenesis (a weak carbonate soil only) and weathering (lack of oxidation), the stratigraphic position relative to underlying dated units in the trench, and lithostratigraphic and soil correlations with Sequence $\mathrm{H}$ in trench SCR-T1 (figs. 7, 8; table 7).

\section{Depositional Rates}

The available age control was used to calculate the average depositional rates for the intervening stratigraphic section between individual dated units. The procedure is probably valid as an approximation for the sandy aggradational sequences of the hangingwall block, because these sediments lack strong soil development or well-developed paleosurfaces indicative of long depositional hiatuses. The depositional rates are in the range of 0.003 to $0.06 \mathrm{~cm} / \mathrm{yr}$. These depositional rates are applied as a means to directly estimate the age of faulted horizons identified in the depositional section between the age control.

\section{STRUCTURAL FEATURES}

\section{General Characteristics}

The Stagecoach Road fault in both trenches is characterized by a wide, complex zone of deformation (figs. 7, 8; pls. 1-3). Major displacement typically is centered on a main fault zone, which is defined as the prominent set of fractures and shears separating hanging-wall and footwall units. This fault zone is typically 2 to $6 \mathrm{~m}$ wide and commonly contains multiple fault strands (figs. 7,8). Secondary deformation manifested by fracturing, graben formation, and backtilting of units in the hanging wall can be found locally as much as $12 \mathrm{~m}$ west of the main fault (figs. 7, 8; pls. 1-3). In trench SCR-T1, footwall deposits also are extensively sheared and fractured 15 to $20 \mathrm{~m}$ east of the primary fault. However, the absence of deformation in trench SCR-T2 indicates no significant
Quaternary activity on a fault strand mapped by Simonds and others (1995) to the east of trench SCR-T1. The footwall block lacks significant deformation east of the main fault zone in trench SCR-T3.

\section{Primary Faulting}

\section{Fault Geometry}

The fault zone in trench SCR-T1 is defined by 6-m-wide zone of anastomosing shears and fractures that typically is well cemented by laminae of carbonate and silica (figs. $6 B$ and $7 ;$ pl. 2). A central zone of closely spaced shears is 35 to $60 \mathrm{~cm}$ wide and typically strikes N. $0^{\circ}$ to $20^{\circ} \mathrm{W}$. and dips $75^{\circ}$ to $85^{\circ} \mathrm{W}$. Many individual fractures adjacent to this zone dip steeply eastward in the hanging wall and steeply to moderately westward in the footwall. This imparts an upward-flaring geometry to the fault zone resembling a pseudo flower-type structure. The absence of the fault in the first borehole drilled $50 \mathrm{~m}$ west of the fault requires that the dip of the fault exceeds $60^{\circ}$ to at least $85 \mathrm{~m}$ depths. Another distinct strand of the fault zone juxtaposes different bedrock units (Tv1, Tv2) in the footwall block, $20 \mathrm{~m}$ to the east of the main zone (pls. 1, 2). This fault is associated with 0.75 -m-high vertical step in the upper bedrock contact. The $20-\mathrm{m}$ down-to-the-west difference in the depth to bedrock in the two boreholes drilled in the hanging-wall block west of the fault zone in trench SCR-T1 is consistent with an intervening buried fault strand that is not exposed at the surface.

The main fault zone in trench SCR-T3 is comprised of four separate strands, spaced approximately 0.5 to $1 \mathrm{~m}$ apart (fig. 8; labeled faults $A$ to $D$, from east to west on pl. 3). These fault strands individually strike N. $20^{\circ}$ to $45^{\circ} \mathrm{E}$. and dip $55^{\circ}$ to $65^{\circ} \mathrm{W}$., vary from $10 \mathrm{~cm}$ to $1 \mathrm{~m}$ in width, and typically increase in structural complexity upsection.

\section{Structural Fabric}

The dominant structural fabric of the fault zone is a complicated network of fractures and shears coated by multiple laminae of carbonate and (or) opaline silica (fig. 6). The thickness of component fractures is variable but commonly falls within a range of $\leq 1$ to $5 \mathrm{~cm}$. Fault deformation in loose, friable sand commonly lacks carbonate cement but instead is 
expressed as finely etched, but uncoated, sets of fractures and shears.

Numerous gravel clasts within and adjacent to fractures and shears are aligned subparallel to the fracture fabric. This tectonic imbrication commonly results from the primary orientation of clasts deposited adjacent to surface ruptures as part of scarp-derived colluvial wedges (Swan and others, 1980; Crone and others, 1987; Nelson, 1992).

\section{Fissures}

At several stratigraphic horizons, lithologic units and soils near the fault zone are disrupted by narrow vertical or steeply dipping zones filled with variably cemented mixtures of sand, silt, and gravel. These features are interpreted as debris-filled fissures that open between or adjacent to shears and fractures during surface rupture (Nelson, 1992). The boundary fractures of these fissures are sometimes coated with thin, carbonate laminae, which are particularly evident where plated against the shear zone on the footwall side. Several of these filled fissures appear to underlie scarp-derived colluvial wedges.

\section{Slip Orientations}

Previous surface mapping along the Stagecoach Road fault identified evidence for leftlateral components of slip in the form of slickenline orientations on bedrock fault exposures and possible left-lateral stream deflections (O'Neill and others, 1992; Simonds and others, 1995). Orientation data suggestive of lateral slip have not been observed in any of the Stagecoach Road trench exposures. A bedrock fault surface within fault strand $A$ at the base of trench SCR-T3 contains well-defined slickenlines with essentially a dip-slip orientation (with a trend of N. $80^{\circ} \mathrm{W}$. and a plunge of $50^{\circ}$, on a fault surface of N. $12^{\circ}$ E., $52^{\circ}$ NW.). Striae with similar dip-slip orientations are developed on another bedrock exposure of the same fault in a gully to the south of the trench. Unambiguous slip-orientation data were not observed in trench SCR-T1.

\section{Secondary Deformation}

Deposits adjacent to the fault zone are deformed within the hanging-wall blocks in both trenches and in the footwall block between the main and eastern strands of the fault in trench SCR-T1 (figs. 7, 8; pls. 1-3). The dominant components of this secondary deformation are fracturing, stratal backtilting, and development of antithetic grabens. In both trenches most of this deformation is concentrated in the hanging-wall blocks.

\section{Fracturing}

The dominant expression of this deformation is numerous isolated or clustered fractures with little or no detectable displacement of strata. The fractures are 1-10 mm thick and commonly are filled discontinuously with carbonate. Fracture orientations are highly variable, with strikes generally subparallel or oblique $\left( \pm 20^{\circ}\right)$ to the primary fault trace and generally steep dips oriented both synthetic and antithetic to the main fault zone.

\section{Upward Terminations}

Most of the secondary fracturing seems to be confined to deposits within the lower one-half of the exposed hanging-wall blocks. In both trenches, sets of fractures terminate at the base of stratigraphic horizons that are commonly associated with scarpderived colluvial wedges related to individual faulting events (the upper contact of Unit E3b in trench SCR-T1, or the top of Unit H3c in trench SCR-T3; figs. 7, 8, pls. 2, 3). These upward fracture terminations also coincide in places with other types of secondary deformation, including abrupt upsection decreases in the amount of eastward backtilting or discrete offsets of units on the western boundary faults of the antithetic grabens. Upward terminations of secondary fractures represent an important criterion for identifying the stratigraphic position of individual faulting events in both trenches.

\section{Backtilting of Strata}

Lower stratigraphic units are backtilted to the east against the fault zone in the hanging-wall blocks of both trenches. The amount of stratal backtilting near the fault zone appears to progressively decrease upsection, with average dips varying from about $10^{\circ} \mathrm{E}$. near the base of the trenches to subhorizontal at bench level. Slightly deformed or undeformed units in the upper part of the hanging-wall blocks dip about $5^{\circ}$ to $10^{\circ} \mathrm{NW}$., subparallel to the westward slope of the modern land surface. Backtilting also increases in magnitude eastward along a given tilted horizon, reaching maximum values adjacent to the main fault. 


\section{Development of Antithetic Grabens in Hanging Walls}

The eastward backtilting of stratigraphic units in the hanging walls of both trenches is associated with the formation of asymmetric antithetic grabens adjacent to the fault zone (figs. 7,8 ; pls. 2, 3).

Discrete graben structures are visible only in the lower parts of the hanging wall, although immediately overlying units dip slightly eastward, indicating that the graben may affect units in the upper part of the trenches as well. The graben in trench SCR-T3 averages $14 \mathrm{~m}$ in width and is bounded to the west by a distinct subsidiary fault zone, $20-35 \mathrm{~cm}$ wide, with a 37-cm down-to-the-east vertical displacement of Unit F3 (fig. 8; pl. 3). Trench SCR-T1 contains a much narrower (2-3 m wide) graben localized near the primary fault zone. The western border of this feature is defined by a zone of distributed fracturing, flexuring, and local fissuring, and units near the base of the trench are displaced $16 \mathrm{~cm}$ down to the east across several shears. Most of the secondary fracturing in the hanging walls is concentrated in the graben interiors, and particularly within or near the boundary structures. The positions and geometries of grabens and flexures are consistent with formation in response to localized distention and collapse of the hanging wall above the westward-dipping fault zone.

\section{PALEOSEISMIC INTERPRETATIONS}

\section{Number and Amounts of Surface-Faulting Events}

The relationships between lithologic units and deformation exposed in trench walls are used to interpret the number and amounts of displacements associated with individual surface ruptures on the Stagecoach Road fault zone (tables 8, 9). The evidence for identifying specific events typically are localized at discrete lithologic contacts (event horizons) that are assumed to correspond to the land surface at the time of surface rupturing associated with paleoearthquakes (Swan and others, 1980; Allen, 1986; Schwartz and Coppersmith, 1986; Machette, 1988; Schwartz, 1988; Nelson, 1992). Event horizons thus mark the stratigraphic position of interpreted faulting events.

Table 8. Criteria used to interpret surface-faulting events in trenches SCR-T1 and SCR-T3

[Event horizons shown in figures 9 and 10 ; dashes (--), no data]

\begin{tabular}{|c|c|c|c|c|c|}
\hline Faulting event & Criteria $^{1}$ & Reliability $^{2}$ & $\begin{array}{c}\text { Event } \\
\text { horizon } \\
\text { (top of unit) }\end{array}$ & $\begin{array}{l}\text { Colluvial } \\
\text { wedge }\end{array}$ & Fissure \\
\hline \multicolumn{6}{|l|}{ SCR-T1 } \\
\hline $\mathrm{Z}$ & $\mathrm{D}, \mathrm{W}$ & $\mathrm{H}$ & $\mathrm{G} 2 \mathrm{~b}$ & G3 & -- \\
\hline $\mathrm{Y}$ & $\mathrm{W}, \mathrm{F}(?)$ & $\mathrm{H}$ & F3a & $\mathrm{G} 2 \mathrm{a}$ & $\mathrm{G} 1$ \\
\hline$X$ & $\mathrm{U}, \mathrm{R}, \mathrm{G}(?), \mathrm{W}(?)$ & M & E3b (east) & F1 & - \\
\hline W & $\mathrm{U}, \mathrm{R}, \mathrm{G}, \mathrm{W}(?)$ & M & E1 & $\mathrm{E} 2$ & -- \\
\hline V/U (footwall) & $\mathrm{D}, \mathrm{F}(?)$ & M & $\mathrm{A} 1, \mathrm{~A} 2, \mathrm{Tv} 2$ & -- & Bla (part) \\
\hline \multicolumn{6}{|l|}{ SCR-T3 } \\
\hline $\mathrm{Z}$ & $\mathrm{D}, \mathrm{W}, \mathrm{F}$ & $\mathrm{H}$ & 13 & $\mathrm{I} 4 \mathrm{~b}, \mathrm{~J} 2, \mathrm{J3}$ & $14 \mathrm{a}$ \\
\hline $\mathrm{Y}$ & $\mathrm{U}, \mathrm{W}$ & $\mathrm{H}$ & $\mathrm{H} 3 \mathrm{c}$ & $\mathrm{Ila}, \mathrm{Ilb}$ & -- \\
\hline$X$ & $\mathrm{U}, \mathrm{R}, \mathrm{W}(?)$ & M & $\mathrm{H} 1$ & $\mathrm{H} 2 \mathrm{a}, \mathrm{H} 2 \mathrm{~b}$ & -- \\
\hline W & $\mathrm{R}, \mathrm{W}(?)$ & $\mathbf{P}$ & $\mathrm{G} 2$ & G3 & -- \\
\hline V (W-graben only) & $\mathrm{G}, \mathrm{D}, \mathrm{W}$ & $\mathbf{H}$ & F4 & F5 & - \\
\hline $\mathrm{U}$ (footwall) & $\mathrm{U}, \mathrm{F}(?)$ & M & D3 & -- & D3 (part) \\
\hline $\mathrm{T}$ (footwall) & $\mathrm{D}, \mathrm{W}(?), \mathrm{F}$ & $\mathbf{H}$ & A3 & -- & Bla (part) \\
\hline
\end{tabular}


Table 9. Estimates for apparent displacements per event for the Stagecoach Road fault in trenches SCR-T1 and SCR-T3

[cm, centimeters; dashes $(--)$, no data; N/A, not applicable]

\begin{tabular}{|c|c|c|c|c|c|c|c|c|}
\hline \multirow{2}{*}{ Event $^{1}$} & \multicolumn{6}{|c|}{$\begin{array}{l}\text { Apparent displacements per event } \\
\text { (cm) }\end{array}$} & \multirow{2}{*}{$\begin{array}{c}\begin{array}{c}\text { Net apparent } \\
\text { displacements } \\
\text { per event } \\
\text { (cm) }\end{array} \\
\begin{array}{c}\text { Preferred } \\
\text { range }\end{array}\end{array}$} & \multirow[b]{2}{*}{ Value } \\
\hline & $\begin{array}{c}\text { Wedge } \\
\text { thickness }\end{array}$ & $\begin{array}{c}\text { Adjusted } \\
\text { wedge } \\
\text { thickness }\end{array}$ & $\begin{array}{l}\text { Stratigraphic } \\
\text { separation }^{6}\end{array}$ & $\begin{array}{l}\text { Measured } \\
\text { displace- } \\
\text { ment }^{7}\end{array}$ & $\begin{array}{l}\text { Preferred } \\
\text { range }^{8}\end{array}$ & Value & & \\
\hline \multicolumn{9}{|l|}{$\overline{\mathrm{SCR}-\mathrm{T} 1}$} \\
\hline$Z$ & $40-45$ & $50-90$ & $62-82$ & $40-45$ & $40-50$ & 40 & $40-50$ & 40 \\
\hline $\mathrm{Y}$ & $34-42$ & $43-84$ & $38-69$ & -- & $40-85$ & 50 & $34-70$ & 42 \\
\hline $\mathrm{X}$ & $15-24$ & $19-48$ & $81-105$ & -- & $30-80$ & 50 & $28-75$ & 47 \\
\hline W & $28-33$ & $35-66$ & $80-88$ & -- & $40-80$ & 60 & $34-67$ & 51 \\
\hline W (graben) & N/A & $\mathrm{N} / \mathrm{A}$ & N/A & 16 & $15-20$ & 15 & N/A & N/A \\
\hline V/U (foot-wall) & -- & -- & N/A & -- & $80-135$ & 100 & N/A & N/A \\
\hline \multicolumn{9}{|l|}{ SCR-T3 } \\
\hline $\mathrm{Z}$ & $60-80$ & $75-160$ & $97-110$ & 100 & $100-110$ & 105 & $41-45$ & 43 \\
\hline $\mathrm{Y}$ & $25-50$ & $31-100$ & $60-100$ & -- & $60-100$ & 75 & $47-79$ & 59 \\
\hline $\mathrm{X}$ & $30-40$ & $38-80$ & $80-103$ & -- & $60-80$ & 70 & $49-65$ & 57 \\
\hline W & $38-53$ & $48-106$ & $103-130$ & -- & $65-110$ & 100 & $44-74$ & 67 \\
\hline V (graben) & $16-28$ & $20-56$ & $\mathrm{~N} / \mathrm{A}$ & 37 & $30-40$ & 35 & $N / A$ & N/A \\
\hline $\mathrm{U}$ (foot-wall) & -- & -- & -- & - & -- & -- & N/A & N/A \\
\hline $\mathrm{T}$ (foot-wall) & -- & - & $56-90$ & 68 & $55-75$ & 70 & N/A & N/A \\
\hline
\end{tabular}

TFulting events listed in table 9.

${ }^{2}$ Apparent fault displacements for individual events, measured parallel to dip of fault, using various methods.

${ }^{3}$ Net apparent fault displacements for individual events, adjusted from preferred values for apparent displacement in preceding column by removing effects of local deformation (primarily backtilting and graben formation) in hanging-wall block, using projection techniques described in text.

${ }^{4}$ Fault displacement derived from thickness of interpreted scarp-derived colluvial wedges, which typically represents a minimum value of the displacement per event.

${ }^{5}$ Fault displacements derived from adjustments of the thickness of scarp-derived colluvial wedges. Corrections are applied assuming the wedge thickness represents 50 to 80 percent of the amount of displacement per event, as discussed in text.

${ }^{6}$ Fault displacements derived from the stratigraphic separation between successive event horizons, which assumes that this entire thickness represents a scarp-derived colluvial wedge with no inter-event burial of the fault trace.

${ }^{7}$ Displacements per event measured directly from correlative marker horizons offset across the fault.

${ }^{8}$ Preferred values for apparent displacements per event, typically involving judgement in removing extreme values, and emphasis on best measurement data. Both ranges and single value estimates are given.

\section{General Methods}

\section{Identification of Individual Faulting Events}

Individual faulting events and event horizons are difficult to recognize in the trenches because the footwall block generally does not contain units correlative with faulted strata on the hanging-wall block. This precludes use of one of the most reliable means of identifying faulting events, which is the incremental downsection increases in the amount of displacement of specific stratigraphic horizons. Other, somewhat more ambiguous, criteria were used to identify surface-rupturing events in both trenches. In general order of reliability and importance, these include (a) a few discrete displaced horizons present on both sides of the fault; (b) upward termination of fractures, fissures, or shears at the upper contact of a stratigraphic unit; (c) abrupt increase at a given stratigraphic interval in the amount $\left(\geq 5^{\circ}\right)$ of eastward backtilting of units towards the main fault zone; and (d) the presence of buried deposits or features; commonly associated with surface ruptures above a given lithologic contact. These latter deposits and features include scarp-derived colluvial wedges deposited against fault traces, buried paleoscarp surfaces developed across fault zones, debris-filled fissures along fault surfaces, and clasts in units above 
stratigraphic horizons that appear tectonically tilted or disturbed adjacent to the fault. Multiple criteria are used to identify events as means of cross-checking results (table 8 ).

There are some inherent uncertainties in identifying rupture events at each trench. Most of these uncertainties involve difficulties in the mapping and tectonic interpretation of specific structures, units, and contacts, such as indistinct or poorly exposed fracture terminations and poorly defined colluvial wedges within sand-dominated stratigraphic intervals in the hanging-wall blocks. Table 8 lists all interpreted surface-faulting events in both trenches, including several ambiguous, but plausible, events recognized near the base of the trench. The table presents the specific criteria and degree of certainty associated with the identification of each event. Both the maximum and minimum numbers of interpreted events are used as input for the calculations of apparent displacements per event, recurrence intervals, and fault-slip rates, although a preferred interpretation is presented that best fits all available data.

\section{Measurement of Apparent Fault Displacements}

Several factors complicate measurement of fault displacements on the Stagecoach Road fault. In both trenches, the main fault zone structurally juxtaposes different units in the hanging-wall and footwall blocks. With a few exceptions, this precludes direct measurements of the displaced horizons across the fault. Instead, fault displacements must be estimated indirectly using the thicknesses of scarp-derived colluvial wedges or the amounts of stratigraphic separation between successive event horizons (see below).

Secondly, the geomorphic setting and local stratigraphy of both trenches indicate a potential for the burial of fault-generated colluvial wedges by deposition across the fault (eolian mantles and slope wash) between surface-faulting events. For example, Sequence $\mathrm{H}$ depositionally overlaps the most recent rupture in trench SCR-T1. This factor introduces the difficult task of separating tectonically induced wedges from nontectonic burial units on the hanging wall.

The footwall block adjacent to the fault probably has experienced an unknown amount of stripping and erosion during fault-scarp degradation between surface ruptures. Some footwall erosion is indicated by the absence of the A or B soil horizons associated with near-surface carbonate horizons on the footwall blocks of both trenches. The net amount of stripping is impossible to determine directly but probably does not greatly exceed the likely thickness of the soil epipedon (upper 0.5 to $1 \mathrm{~m}$ ), as the upper laminar caps of the $K$ horizon are still present beneath upper Pleistocene to Holocene colluvium adjacent to the fault.

For the above reasons, most of the individual and cumulative amounts of surface displacements are not true measurements of actual fault displacements. Rather, they represent estimates of the dip-slip separation of hanging-wall units inferred from the observed stratigraphic relationships across the fault. Therefore, we have applied several different measurement techniques that are designed to provide lower and upper bounds for the estimates of fault separations, herein referred to as apparent displacements.

The apparent displacements of individual surface-rupturing events are estimated in three basic ways. Lower-bound estimates are derived from the thicknesses of scarp-derived colluvial wedges (table 9). However, paleoseismic studies at other sites at Yucca Mountain, as well as elsewhere, suggest that wedge thicknesses may underestimate the actual surface displacements by 50 to 80 percent, owing to factors such as (a) partial burial of the free face following rupture; (b) problems in identifying the complete depositional wedge, including fine-grained wash facies, of the fault scarp; and (c) erosional beveling of the rupture footwall during fault scarp formation (Wallace, 1977; Swan and others, 1980; Machette, 1988; Machette and others, 1992; Nelson, 1992; Menges and others, 1994). Values shown in table 9 incorporate uncertainties in identifying and measuring the thickness of colluvial wedges, particularly in fine-grained sandy units.

Two types of upper-bound estimates of apparent displacements per event have been developed (table 9). First, the observed thicknesses of the colluvial wedges, measured at a point close to the fault, have been increased by factors of 1.25 to 2.0 in order to accommodate the possibility of underestimation of per-event displacements noted above. This adjustment assumes that the small, scarp-derived colluvial wedges identified are debris facies of much larger colluvial wedges, which include the overlying fine-grained sandy deposits as wash facies (for 
example, Units E2, E3a, and E3b in trench SCR-T1, pl. 2; compare Wallace, 1977; Nelson, 1992).

Another upper-bound measure of apparent displacement is provided by the total stratigraphic separation between interpreted event horizons on the hanging-wall block, measured at the fault (table 9). This procedure assumes that the entire separation between the two event horizons on the hanging-wall block is an approximate measure of the apparent displacement associated with the faulting event at the lower horizon. Thus, the entire apparent displacement per event is buried or aggraded completely by postevent deposition on the hanging-wall block. This assumption also requires that the footwall block has neither aggraded or eroded appreciably, while net deposition occurs only on the hanging-wall block in response and proportional to the amount of surface faulting. That is, the depositional rates on the hanging-wall block must be equal to, or less than, displacement rates. This relationship is required in order to provide space for deposition and preservation of downfaulted units in the hanging-wall block, given the absence of net aggradation on the footwall block.

The larger of the two measures, adjusted wedge thickness or stratigraphic separation, is taken as the upper-bound estimate for apparent displacements per event. In either case, most of the sediment between two event horizons represents large, scarp-derived colluvial wedges.

Table 9 also presents preferred values for the amounts of individual displacements per event at each interpreted event horizon. Most preferred estimates involve a significant amount of judgment that typically is weighted more heavily toward the smaller values of adjusted wedges or toward stratigraphic separation measurements. Net apparent displacements per event are derived from the preferred estimates by removing the effects of backtilting and (or) graben formation adjacent to the fault zone (table 9). This net adjustment is derived from projection of event horizons into the fault zone from undeformed sections of the hanging-wall block and uneroded parts of the footwall blocks. This correction for net apparent displacements tends to reduce the amount of displacement measured at the fault trace (Swan and others, 1980; Schwartz, 1988).

\section{Faulting Events and Apparent Displacements in Trench SCR-T1}

In trench SCR-T1, at least two, and preferably four surface-rupturing events are defined in the hanging wall on the basis of the criteria listed earlier. Two events are interpreted with higher confidence on the upper part of the trench, including the most recent event, Event $Z$, and the penultimate event, Event $Y$ (figs. 7, 9; pl. 2; table 8). Event $Z$ cuts through a preexisting fissure and appears to vertically displace the underlying colluvial wedge from Event $Y$. (Unit $\mathrm{G} 2 \mathrm{a}$ ) by $40 \mathrm{~cm}$. If correct, this is the only place in this trench where the vertical displacement from an event on the fault zone can be directly measured. Event $Z$ also is associated with a small, scarp-derived colluvial wedge (Unit G3). Event $Y$ is interpreted to have occurred at the top of Unit F3a. This event is defined on the basis of (a) upward termination of several carbonate-coated fractures and shears (both secondary and primary) at and west of the fault; (b) deformation of the upper contact of Unit F3a near the fault zone; (c) a possible wedge (Unit G2a); and (d) a carbonate-coated fissure filled with Unit G1. There is a minor, albeit undiagnostic, amount of eastward tilting of Sequences $G$ and $F$, which also supports the proposed events in this stratigraphic interval.

Additional events are necessary lower in the trench to incrementally increase the amount of backtilting of progressively older strata eastward into the fault zone, as well as create space for the depositional sequences against the fault zone (figs. 7,$9 ; \mathrm{pl} .2$; table 8). The lower part of the hanging-wall block beneath the bench contains many carbonate-coated fractures, which cannot be mapped to continue upwards into overlying units, thereby strongly suggesting at least one, and preferably two, earlier faulting events. However, the exact stratigraphic position of these events are more difficult to define in this part of the trench. One surface rupture (Event X) is indicated near the top of Sequence $E$ by (a) the apparent upward truncation of numerous fractures beneath Sequence $F$ in a poorly exposed area near the bench, (b) a slight increase in backtilting at the top of Unit E3b, and (c) an indistinct colluvial wedge (Unit F1) deposited above Unit E3b at the fault. A possible fissure filled with disseminated ash may continue downward from this horizon along the western border fault to the antithetic graben as well. 


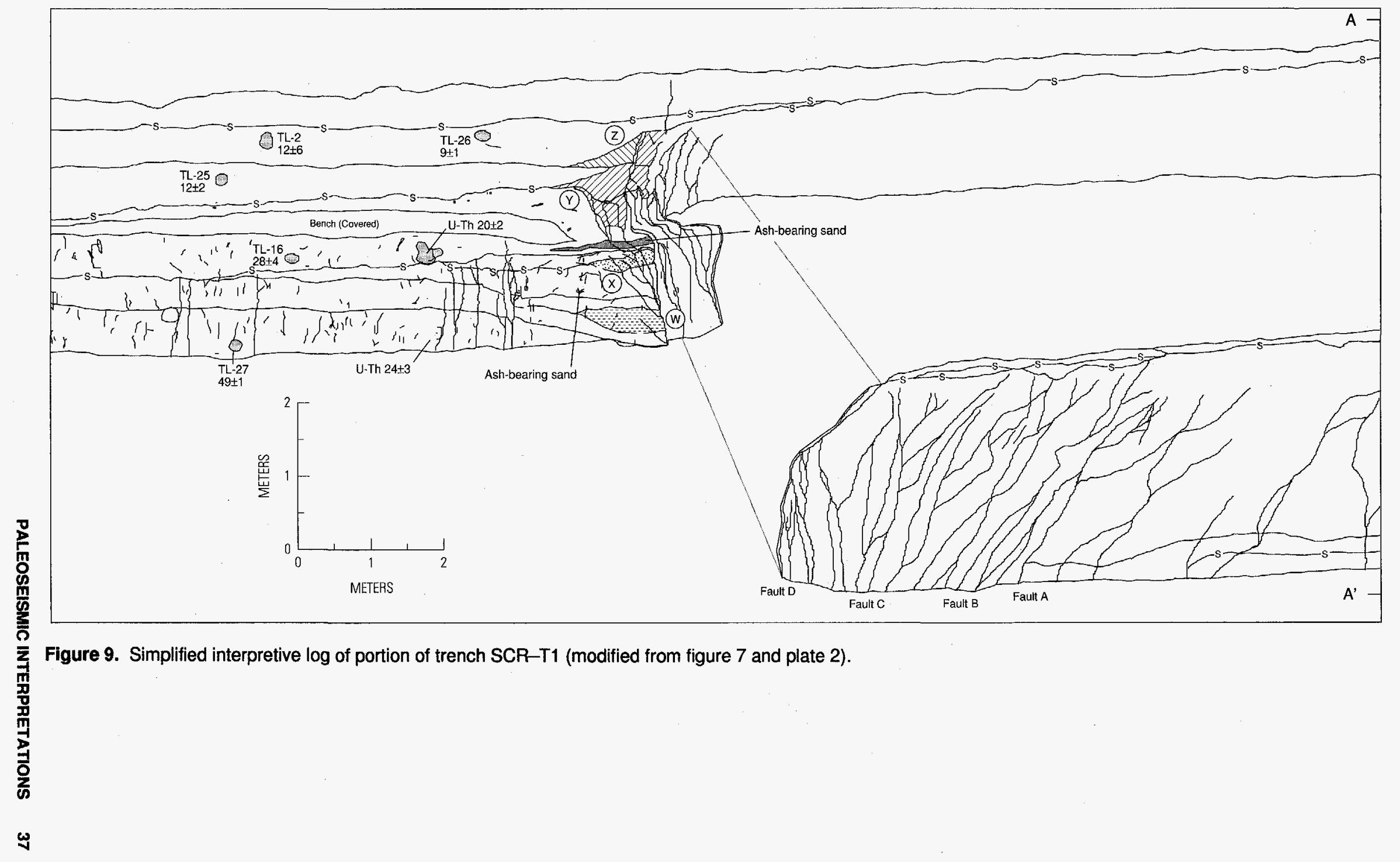


Another event horizon (Event W at the top of Unit E1) is indicated by the apparent termination beneath Unit E3b of a discrete $16-\mathrm{cm}$, down-to-the-east offset of underlying units in Sequence $D$ across the western boundary fault of the graben. This event horizon is associated with a sharp increase in the eastward tilt of Unit E1 and Sequence D, as well as a poorly-defined lenticular unit (Unit E2) that may be related to scarpderived colluvial deposition.

Near the top of the trench wall, a thin fracture with no carbonate coatings and no detectable offset continues upward from the fault zone through overlying units of Sequence $\mathrm{H}$ essentially to the base of the surface vesicular (Av) horizon (fig. 7; pl. 2; table 8). The tectonic significance of this fracture is unclear, but it is not unequivocally associated with movement on Stagecoach Road fault. This fracture may be related to ground shaking from seismic events on other nearby faults, or it may be associated with nontectonic pedogenic processes.

Another episode of faulting is present on the eastern strand of the fault zone in the middle of the footwall block in trench SCR-T1 (pls. 1, 2). The amount of bedrock displacement cannot be determined because two different bedrock units (Tv1 and Tv2) are juxtaposed across this strand of the fault in the trench wall. However, the bedrock erosional surface developed across the top of these units is displaced $70 \mathrm{~cm}$ down to the west across the fault. This displacement probably represents at least one surfacerupturing event (event $\mathrm{V} / \mathrm{U}$ ) during the depositional interval of the overlying colluvial-alluvial unit (Unit B1), based on (a) pronounced thickening of the basal section of B1a across the bedrock fault step, probably through deposition of a colluvial wedge obscured by subsequent pedogenesis; (b) scarplike beveling of upper Tv1 contact at the eastern (footwall) side of fault; and (c) possible development of debrisfilled fissure unit along the fault contact between Units Tv2 and Tv1. Details of this interval of surface faulting are difficult to resolve because of overprinting by pedogenic carbonate.

Although poorly constrained in most cases, several types of measurements of the sizes of individual events on the main fault zone in trench SCR-T1 are listed in table 9. Lower estimates derived from thicknesses of colluvial wedges range between 15 and $45 \mathrm{~cm}$, which includes a true displacement measurement of 40 to $45 \mathrm{~cm}$ from the most recent event. Upper bounds from adjusted wedge thicknesses and stratigraphic separations between event horizons are much larger, in the range of 19 to $105 \mathrm{~cm}$. The preferred interpretations of event sizes range from 30 to $85 \mathrm{~cm}$, and net apparent displacements range from 28 to $75 \mathrm{~cm}$.

These displacement amounts are derived using a preferred four-event scenario in the hanging wall. Reduction of the number of events potentially could increase the upper bound estimate of displacements per event derived from stratigraphic thickness; most of the increase is concentrated in the lower section if the older, less well-defined events are omitted. Apparent separations derived from the thicknesses of colluvial wedges would provide more minimum estimates if fewer events are interpreted.

\section{Faulting Events and Apparent Displacements in Trench SCR-T3}

At least three, and preferably five faulting events are indicated in the hanging-wall block adjacent to the main fault zone in trench SCR-T3 (figs. 8, 10; pl. 3; table 8). The event sequence appears generally similar to that in trench SCR-T1 with the addition of one more event, although stratigraphic relationships for most faulting events typically are better expressed in trench SCR-T3. Surface-rupturing events are interpreted in trench SCR-T3 on the basis of similar criteria used in trench SCR-T1: upward fracture terminations, progressive backtilting, and variably distinct colluvial wedges and (or) related fissures.

Two well-defined events are interpreted on the upper part of trench SCR-T3 (figs. 8, 10; pl. 3; table 8 ). The most recent event (Event $Z$ ) occurred on fault $\mathrm{C}$ and is represented by a large gravel wedge, with a basal gravelly and upper sandy facies (Units I4b, J2, and J3), that was deposited against a steeply dipping scarp free face. This composite wedge overlies a wide, debris-filled fissure (Unit I4a). The penultimate event (Event $\mathrm{Y}$ ) is identified just above the bench on fault $D$ at the top of Unit H3c. Evidence for Event $Y$ consists of (a) upward truncation of faults and fractures at the base of Unit $\mathrm{I} 2$ at the western margin and within the antithetic graben; (b) apparent deformation, particularly evident in the carbonate soil, of Unit H3; and (c) a possible scarp-derived colluvial wedge (Unit Ila and Unit I1b).

One to two possible additional events are suggested at bench level and near the base of the 


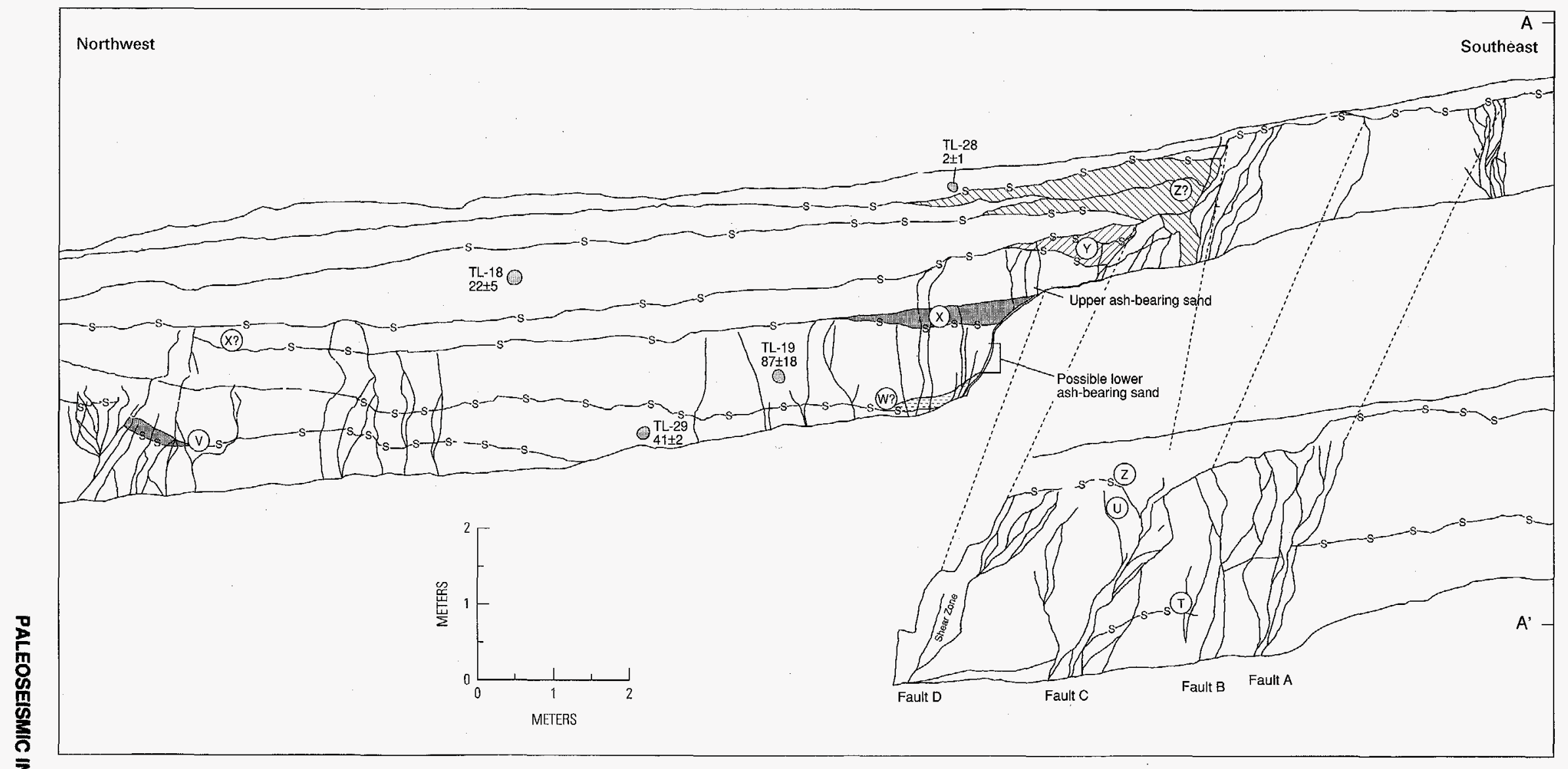

Figure 10. Simplified interpretive log of portion of trench SCR-T3 (modified from figure 8 and plate 3). 
trench on the westernmost fault strand (figs. 8 and 10; fault $\mathrm{D}$, pl. 3 ; table 8 ) by progressive backtilting of units within the antithetic graben and by the presence of possible scarp-derived colluvial wedges. However, these events are less well-defined than those higher in the trench. One possible event (Event $\mathrm{X}$ ) is suggested by an indistinct colluvial wedge (Units $\mathrm{H} 2 \mathrm{a}, \mathrm{H} 2 \mathrm{~b}$ ) combined with apparent truncation of secondary fractures below Unit H3c. The greater eastward dips of units in Sequence $G$ near the base of the trench may represent another possible event horizon (Event W). This event possibly is associated with an ambiguous wedge-like unit (G3); however, the apparent backtilting alternatively may reflect burial of a large colluvial wedge associated with an event (Event V) inferred beneath the present floor of the trench (see below).

A thin, uncoated fracture with no displacement continues upward from fault $\mathrm{C}$ through the unfaulted colluvial wedges ( $14 \mathrm{~b}, \mathrm{~J} 2$ and $\mathrm{J} 3$ ) and an overlying deposit (J4) to the base of the surface Av horizon. The morphology and stratigraphic position of this feature are similar to the fracture at the top of trench SCR-T1 and thus are interpreted in similar fashion. This fracture probably is not related to movement of the underlying fault.

A fifth event (Event $V$ ) is recognized in the hanging-wall block of the western boundary fault of the graben. This event is identified confidently on the basis of displacement and tilting of Unit F4 and older units, a possible small scarp-related colluvial wedge (Unit F5), and fault disruption and fracturing of Unit F4 on the downthrown block at the western edge of the graben (pl. 3; figs. 8, 10; table 8). Sequence F disappears to the east beneath the floor of the trench before intersecting the main fault zone (fault $D$ ) as a result of eastward backtilting within the graben. However, the displacements of Sequence F on the western graben-bounding fault and the pronounced rotation of the units in the lower part of the graben must be associated with a relatively large event on the main fault that is not exposed in the trench. At least one possible small event recorded in deposits at or near the bench on the western graben-bounding fault may correlate with Event $\mathrm{X}$ at the top of Unit $\mathrm{H} 1$ on the main fault (figs. 8, 10; pl, 3).

Two much older faulting events (Events $U$ and T) are interpreted on the eastern strands of the main fault zone (faults $\mathrm{A}, \mathrm{B}$, and $\mathrm{C}$ ) in the footwall block (figs. 8,10 ; table 8 ; pl. 3). Event $U$ is indicated by a carbonate-cemented disrupted section, possibly related to fissure development within Unit D1 located to the west of the most recent rupture on fault $C$. Event $T$ is expressed as apparent disruption of unit $\mathrm{B} 1 \mathrm{a}$, probably associated with fissure development, west of fault $B$. This event appears to postdate beveling of Unit Bla and was subsequently buried by a sandy colluvial unit (Unit B1c) that may be fault related. Event T' probably is responsible for the separation of underlying Units A1 to $A 3$ on both faults B and A. The footwall events $\mathrm{U}$ and $\mathrm{T}$ seem temporally and spatially disconnected from the main sequence of rupture events on the western fault trace (fault D), in that Event $T$ occurred prior to deposition of Sequence $D$ at the top of the footwall block and both events predate most of the carbonate accumulation in the well-developed relict soil on Unit D. However, most of the large displacement of footwall Sequences $A$ to $D$ on fault $C$ in the south wall is attributed to the most recent event (Event $\mathrm{Z}$ ) identified on the north wall of the trench. There is an additional small displacement (about $10 \mathrm{~cm}$ ) at the base of Unit $\mathrm{C} 2$ on fault $\mathrm{A}$, above the proposed event horizon $\mathrm{T}$. This reflects a component of faulting on this strand associated with either Event $U$ or one or more of the younger everits recognized on the hanging wall.

Lower- and upper-bounding values for the apparent displacements of individual events at the fault zone are derived in similar fashion to SCR-T1 (table 9) using the preferred five-hanging-wall-event scenario for this trench. Lower-bound estimates are derived from the thicknesses of colluvial wedges, which range from 25 to $80 \mathrm{~cm}$. The large value is associated with a composite wedge from the most recent event (Event $Z$ ). The corresponding ranges of upper-bounding values from the adjusted wedge thicknesses and stratigraphic separations are 31 and $160 \mathrm{~cm}$, respectively. The preferred estimates of displacements per event range between 60 to $110 \mathrm{~cm}$, values that are much closer to the wedge-derived measurements. However, the net apparent displacements per event are somewhat smaller, in the range of 41 to $79 \mathrm{~cm}$. Unit D3 is displaced $100 \mathrm{~cm}$ across fault $\mathrm{C}$ on the north wall of the trench. Most of this offset is attributed to the most recent event (Event $Z$ ), although an unknown, but probably small amount may be related to event $\mathrm{U}$.

Reduction in the number of events will have the same effect on apparent separation estimates described previously for trench SCR-T1. Elimination of event 
W particularly will increase the displacement amount for the buried event on the main fault corresponding to event $\mathrm{V}$ on the graben-bounding fault.

\section{Age Constraints on Faulting}

Age control for faulting events is developed from relationships between event horizons and the estimated ages of lithologic units in trenches. These ages are derived from specific geochronologically dated units combined with general soil stratigraphic relationships (see preceding section on "General Characteristics of Trench Stratigraphy;" figs. 7, 8, 11; tables $4,6,8,10)$. None of the ages were collected from deposits such as scarp-derived colluvial wedges or fissure fills that are directly associated with faulting. Instead, dated units occur in stratigraphic sequences above or below the interpreted event horizons, and thus the dated unit provides only minimum or maximum ages for the faulting event. A close temporal association between event horizons and the age control is possible in a few cases where a dated deposit closely overlies or underlies an event horizon with little or no soil development (for example, Unit H1 in trench SCR-T1; fig. 7; pl. 2).

Table 10 and figure 11 present the age control currently available for individual and multiple faulting events discussed previously. The table and figure state the reported ages and analytical errors for dates that occur stratigraphically above and below the events, as well as a more restricted preferred range in ages which incorporates additional geologic or soil-stratigraphic constraints provided by relationships among faulting events, dated units, and pedogenic features such as carbonate deposition. Also included is a third estimate of ages and intervals of faulting events based on the interpolations of depositional rates computed between successive dated units (see preceding section on trench stratigraphy). The depositional rates are used to calculate the ages for event horizons by computing the time required to accumulate the observed stratigraphic thickness between the event horizon and the closest overlying dated deposit and then adding this increment to the age of the control unit. Several paleoseismic studies on the San Andreas fault have used sedimentation rates to additionally constrain paleoearthquake ages (Sieh, 1978; Sieh and others, 1989; Biasi and Weldon, 1994). This method assumes constant depositional rates between dated horizons and thus will work best in the depositional setting of the Stagecoach Road trenches in poorly bedded to massive, sandy units with weak cumulic soils. These values may underestimate the age of event horizons that occur within intervals with buried soil, which reflect intermittent periods of soil formation and episodic deposition.

The best age constraints are available for the four youngest events (Events Z-W) identified in each trench within stratigraphically continuous sections of the hanging-wall block. The three youngest events (Events Z-X) can be easily correlated between trenches. In particular, there is good agreement in correlative trench stratigraphy and age assignments in this interval in each trench. The stratigraphic context of the next oldest events (Event W) in the trenches is also similar at both sites. However, the individual Event $W$ in each trench may not correlate with one another, but instead be two separate and distinct ruptures, based on possible differences in the assigned ages of affected deposits (see discussion below). Thus, in order to avoid confusion in correlating events between the sites, the individual fault chronologies from the trenches were combined into a single composite event chronology that uses a prime notation (that is, $Z^{\prime}, Y^{\prime}, X^{\prime}$...).

The most recent surface-rupturing event $\left(Z^{\prime}\right)$ underlies an unfaulted unit with a latest Pleistocene to early Holocene age (TL-02, $12 \pm 6 \mathrm{ka}$, and TL -26 , $9 \pm 1 \mathrm{ka}$ ) in trench SCR-T1 (figs. 7,9). The weak carbonate soil in the overlying units $(\mathrm{H} 4$ to $\mathrm{H} 1)$ suggests a minimum age of middle to early Holocene (5-10 ka). This event overlies Unit G2b with a TL age of $12 \pm 2 \mathrm{ka}$ (TL-25) in trench SCR-T1 and Unit I3 with a TL age of $22 \pm 5 \mathrm{ka}$ (TL-18) in trench SCR-T3 (figs. $7,8,9,10$ ). In both trenches, uncemented fractures associated with the most recent event cut thin carbonate plating on older fissures and shear surfaces, which are probably associated with the penultimate event. Thus, the most recent event appears to postdate significant carbonate deposition at this stratigraphic level. Collectively, these data indicate that the most recent event on the fault is latest Pleistocene to middle Holocene (5-15 ka) in age. Most likely, the age is closer to $10-15 \mathrm{ka}$, which agrees with the depositionrate calculations and soil stratigraphy (table 10). This age range also is consistent with a 15-ka age from the undeformed rhizolith in the main fault of trench SCR-T1 as well (HD1439, table 6), which likely grew along the fault zone. Fractures cutting up to the base 


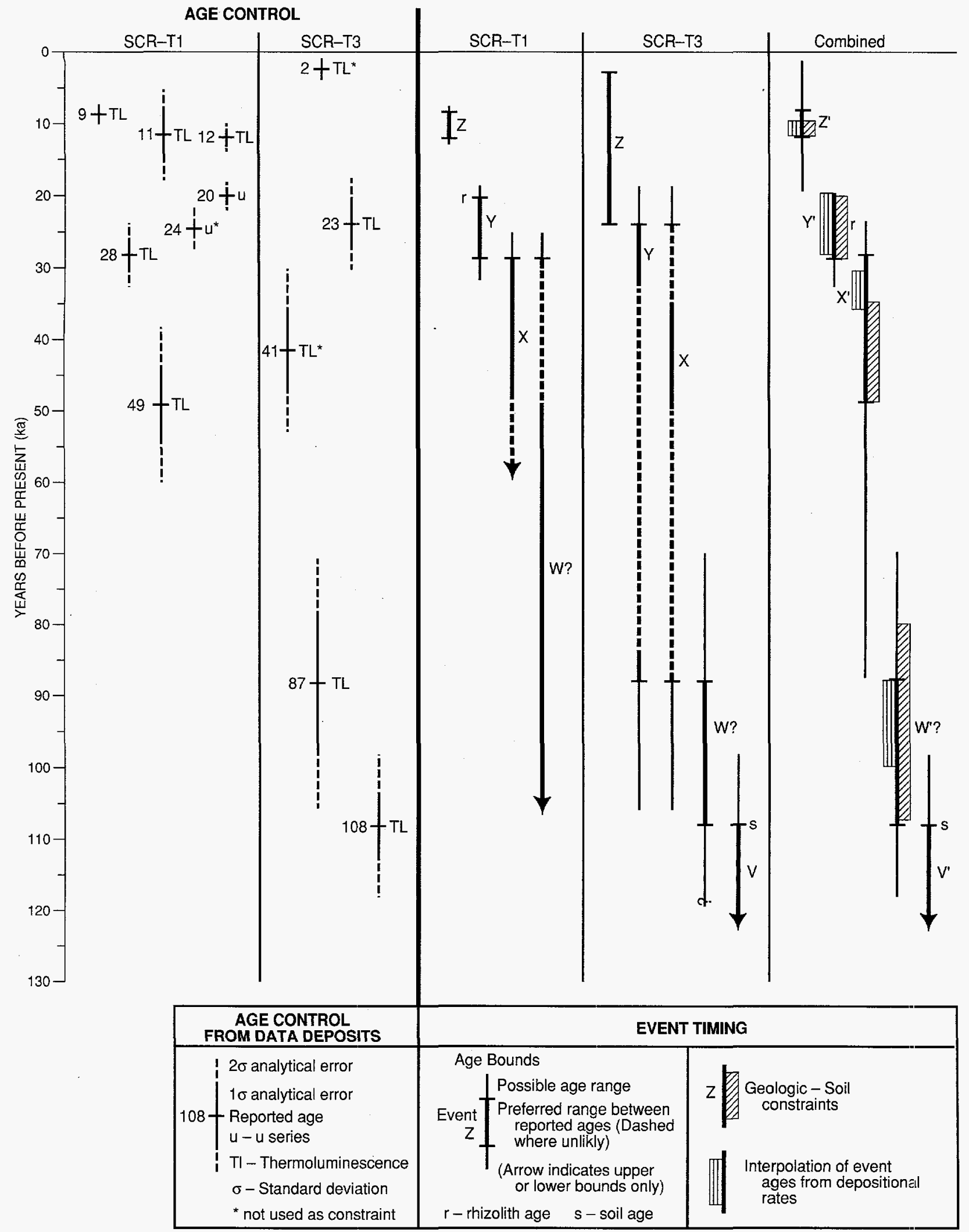

Figure 11. Chart illustrating age control and temporal relationships of faulting events in trenches SCR-T1 and SCR-T3. 
Table 10. Age control for composite set of faulting events identified on Stagecoach Road fault in trenches SCR-T1 and SCR-T3

[Age control derived from dated deposits using geochronologic ages listed in tables 4 and 6 and shown in figures 7 and 8 . Events are queried where uncertain; ka, thousand years ago; ky, thousand years; N/A, not applicable]

\begin{tabular}{|c|c|c|c|c|}
\hline \multirow{2}{*}{$\begin{array}{l}\text { Events and } \\
\text { intervals }^{1}\end{array}$} & \multirow{2}{*}{$\begin{array}{c}\text { Age- } \\
\text { control } \\
\text { alternative }\end{array}$} & \multicolumn{2}{|c|}{$\begin{array}{l}\text { Bounding age } \\
\text { constraints }^{3}\end{array}$} & \multirow{2}{*}{$\begin{array}{c}\text { Estimated } \\
\begin{array}{c}\text { age deposi } \\
\text { tlonal } \\
\text { rates }^{4}\end{array} \\
\text { Range }^{7}\end{array}$} \\
\hline & & $\begin{array}{c}\text { Total } \\
\text { range }^{5}\end{array}$ & $\begin{array}{c}\text { Preferred } \\
\text { range }^{6}\end{array}$ & \\
\hline Events & & \multicolumn{2}{|c|}{ (ka) } & (ka) \\
\hline$Z^{\prime}$ & N/A & $6-14$ & $10-15$ & $10-12$ \\
\hline$Y^{\prime}$ & N/A & $17-32$ & $22-28$ & $19-24$ \\
\hline \multirow[t]{2}{*}{$X^{\prime}$} & A & 24-105 & $35-50$ & $32-36$ \\
\hline & B & $24-53$ & $30-35$ & $26-32$ \\
\hline \multirow[t]{2}{*}{$W^{\prime}(?)$} & A & $69-118$ & $80-110$ & $87-99$ \\
\hline & B & $24-53$ & $40-45$ & $36-48$ \\
\hline $\begin{array}{l}\text { Event } \\
\text { intervals }\end{array}$ & & \multicolumn{2}{|c|}{ (ky) } & (ky) \\
\hline$Z^{\prime}-Y^{\prime}$ & $\mathrm{N} / \mathrm{A}$ & $3-26$ & $10-20$ & $7-14$ \\
\hline$Y^{\prime}-X^{\prime}$ & A & $0-88$ & $10-30$ & $8-17$ \\
\hline$X^{\prime}-W^{\prime}(?)$ & A & $6-77$ & $30-75$ & $51-67$ \\
\hline$Z^{\prime}-X^{\prime}$ & A & $10-99$ & $20-40$ & $20-26$ \\
\hline$Z^{\prime}-W^{\prime}(?)$ & A & $55-112$ & $75-100$ & $75-89$ \\
\hline$Y^{\prime}-X^{\prime}$ & B & $0-36$ & $5-15$ & $2-13$ \\
\hline$X^{\prime}-W^{\prime}(?)$ & B & $0-29$ & $5-15$ & $4-22$ \\
\hline$Z^{\prime}-X^{\prime}$ & B & $10-47$ & $15-25$ & $14-22$ \\
\hline$Z^{\prime}-W^{\prime}(?)$ & B & $10-47$ & $25-35$ & $24-38$ \\
\hline
\end{tabular}

Composite set from both trenches of faulting events listed in table 8 .

${ }^{2}$ Specific set of alternative age-control used in analysis of older events. Alternative A uses the older TL and U-series ages (TL-19 and HD-1447), whereas Alternative B is based on the younger TL ages (TL-27 and TL-29). See text for explanation.

${ }^{3}$ Upper and lower age constraints for faulting events derived from dated deposits in both trenches.

${ }^{4}$ Age ranges for faulting events derived from interpolations using depositional rates computed between dated deposits (see text).

${ }^{5}$ Total age ranges using full error bounds from constraining ages.

${ }^{6}$ Preferred age ranges based on additional constraints from geologic and soil-stratigraphic relationships among faulting events and dated deposits.

${ }^{7}$ Event ages using total range of upper- and lower-bounding age constraints adjusted with interpolations from computed depositional rates between dated deposits (see text).

of the surface Av horizon in both trenches are middle to late Holocene in age, although the features are not associated with detectable fault displacement and may be nontectonic in origin.

This age assignment for the most recent event also is consistent with the morphology of the fault scarp developed across the fault zone at the trenches (fig. 5). The age of the scarp can be crudely estimated by using the technique of Bucknam and Anderson (1979), whereby maximum slope angle is plotted and regressed against log height from scarp profile data. Scarp data from the Stagecoach Road fault plots near the regression line for the Beatty scarp. The latter feature is assigned an approximate $10 \mathrm{ka}$ age (Anderson and Klinger, in press) based on a similar regression analysis and radiocarbon dating of charcoal in associated fluvial gravels.

The faulting event interpreted at the base of the upper wall (Event $Y^{\prime}$ ) deforms the upper horizon of the carbonate soil described in both trenches (Units F3a and $\mathrm{H} 3 \mathrm{c}$ in trenches SCR-T1 and SCR-T3, respectively; figs. 7, 8, 9, 10; tables 7,8 ). Possible associated wedges and fissures contain less pedogenic carbonate. In trench SCR-T1, the event horizon is overlain by deposits of Sequence $G$ that lack significant carbonate and are assigned a latest Pleistocene age based on soil development and a TL age of $12 \pm 2 \mathrm{ka}$ (TL-25; table 4; figs. 7,9). This event horizon underlies the unit with a TL age of $22 \pm 5 \mathrm{ka}$ (TL-18; figs. 8, 10) in trench SCR-T3. A lower constraint in trench SCR-T1 is provided by unit $\mathrm{F} 2 \mathrm{~b}$ below the event horizon with a TL age of $28 \pm 4$ (TL-16; figs. 7, 9). Based on these relationships, a general age range of $15-30 \mathrm{ka}$ is estimated for this penultimate event, with a preferred age bracket of 20-30 ka consistent both with its association with pedogenic carbonate deposition and with depositional rate calculations (fig. 11; table 10).

Any faulting events at mid-bench level, including the proposed Event X, lie immediately below the stratigraphic sequence with the upper rhizolith zone (Sequence F), a TL age of $28 \pm 4 \mathrm{ka}$ (TL-16) in Unit F26, and the upper ash-bearing sand in trench SCR-T1 (Unit F2b) (figs. 7, 9; tables 7, 8). This event horizon underlies a similar ash-bearing sand (H3a) and the unit (I3) with the middle TL age in trench SCR-T3 (TL-18; $22 \pm 5 \mathrm{ka}$; figs. 8,10$)$. In both trenches the event horizon lies within or just below the lower portion of the stratigraphically highest carbonate soil with at least Stage II morphology. This stratigraphic sequence also overlies Unit D1 with the lower TL age of $49 \pm 11 \mathrm{ka}$ (TL-27) in trench SCR-T1, as well as Units G5 and G2 with the TL ages of $87 \pm 18 \mathrm{ka}$ (TL-19) and $40 \pm 12 \mathrm{ka}$ (TL-29), respectively, in trench SC-T3.

The age of the event depends on which of the two age-control alternatives is used in the lower part 
of the trenches (see earlier discussion in section on geochronologic control in trench SCR-T3). In Alternative A, based on the older TL age (TL-19), Event $X^{\prime}$ is assigned a general age range of $25-105 \mathrm{ka}$, but soil-stratigraphic and depositional-rate criteria suggest that the faulting is probably closer to $35-50 \mathrm{ka}$ (fig. 11; table 10). The assigned age is younger in Alternative B, which uses the composite age of $45 \pm$ $8 \mathrm{ka}$ from the two younger TL ages (TL-27 and TL-29), with estimated event ages of 24-53 ka, and preferred values of $30-35 \mathrm{ka}$. As noted previously, Alternative $\mathrm{A}$ is preferred because the older TL age is in good agreement with the U-series age $(108 \pm 10 \mathrm{ka}$; $\mathrm{HD}-1447$ ) of Unit G2 in trench SCR-T3. The Alternative A age control also is in better agreement than Alternative B with the assigned age of the basaltic ash horizon using the most straightforward correlation with air fall ash at the Lathrop Wells source.

The age of faulting near the base of the lower section of the hanging-wall block is more difficult to interpret because of apparent discrepancies in ages of affected deposits between the trenches. This age of uncertainty is added to difficulties in identifying uniquely faulting events in this stratigraphic interval (see earlier section). The stratigraphic contexts of Event $\mathrm{W}$ in both trenches are very similar. That is, the lithostratigraphic units and their relationships to the interpreted event horizon strongly resemble one another in the lower part of each trench. Thus Event W would correlate between trenches. This evidence, combined with the remaining older event in trench SCR-T3 (see below), leads to a simple fiveevent composite scenario for the main sequence of hanging-wall events in both trenches (fig. 11). However, there is a geochronologic problem in this event correlation. Event $\mathrm{W}$ in trench SCR-T1 overlies and thus is younger than Unit D1 with a $49 \pm 11 \mathrm{ka} \mathrm{TL}$ age (TL-27). Event $W$ in trench SCR-T3 underlies and thus must be older than Unit G5 with a TL age of $87 \pm 18 \mathrm{ka}(\mathrm{TL}-19)$ and lies above Unit G2 with a $\mathrm{U}-\mathrm{Th}$ age of $108 \pm 10 \mathrm{ka}$ on pedogenic carbonate (HD1447) and a TL age of $40 \pm 12 \mathrm{ka}$ (TL-29). If these ages are correct and the contradictory young age of TL-29 is in error, the Event W's in each trench must be two temporally distinct events, which creates a six event composite fault chronology for both trenches. This would require also that Event W' (Event W in trench SCR-T1) was not recorded in trench SCR-T3, and that the older event in trench
SCR-T3 was not recorded in trench SCR-T1. The simpler five-event composite scenario is preferred because of: (a) the strong lithostratigraphic and contextual similarity in events between the trenches; and (b) the unlikely prospect of missing events between trenches that are so close together.

This still leaves open the question of which age control alternative to use in assigning an age to Event $W^{\prime}$ in the five-event scenario. In Alternative A with older age control (TL-19 and HD-1447), Event $\mathrm{W}^{\prime}$ is constrained to a broad 69-118 ka age interval with a preferred age of 80-110 ka (fig. 11, table 10). The event is notably younger using Alternative $B$ with the two younger TL ages (TL-27 and TL-29); this alternative establishes a general age of $24-53 \mathrm{ka}$, with preferred values of $40-45 \mathrm{ka}$. Both alternative age assignments are used in subsequent recurrence calculations. However, the Alternative A age control is preferred for reasons outlined previously.

The next oldest event ( $V^{\prime}$ ) in the composite chronology is present only on the graben-bounding fault in trench SCR-T3. This event displaces the top of Sequence $F$. The associated fractures and wedge (Unit F5) contain a similar amount of carbonate cement to the underlying deposits. Assuming Alternative $A$ age control, the age of Sequence $F$ must be greater than the combined 100- to 130-ka age range of the TL and $\mathrm{U}$-series dates from the overlying units in sequence $\mathrm{G}$, and may be $\geq 150 \mathrm{ka}$ based on the degree of soil development.

Little direct age control exists for fault displacements in the footwall blocks in either trench, although there is probably a significant time gap between these events and those on the hanging wall adjacent to the main fault zone. This inference is based on the observation that the soils postdating events are more strongly developed on the footwall blocks, relative to soils on the hanging-wall blocks. Thus these footwall events are not shown in the timing diagram of figure 11. In trench SCR-T1, displacements occur on the eastern fault zone, at or near the base of Sequence B where it thickens across the offiset in the bedrock surface (pl. 2). Fractures and stratigraphy related to these events are overprinted by, and thus predate, the thick, very well developed petrocalcic soil in Sequence B, which indicates that the deformation is probably at least middle to early Pleistocene in age. Most of the older faulting (Events $\mathrm{U}$ and $\mathrm{T}$ ) identified on eastern footwall strands of the fault zone in SCR-T3 (figs. 8, 10; pl. 3) occurred prior to formation 
of the petrocalcic soil in Sequence D, and thus both events are probably at least middle Pleistocene in age.

\section{Paleoearthquake Recurrence}

Recurrence intervals are defined as the time intervals between successive surface-rupturing paleoearthquakes. Two types of recurrence intervals are developed for the composite fault chronology interpreted from the hanging-wall stratigraphy of both trenches (table 11). The intervals between individual events are calculated for certain pairs of successive events with suitable age control, and average recurrence intervals are computed from age control that encompasses more than two faulting events. Both recurrence estimates, which use the same age constraints described herein for individual faulting events, are beset by uncertainties regarding.the commonly indirect, bracketing relationship between specific dated deposits and a given faulting event. This affects the number of inter-event intervals used to compute recurrence intervals. That is, the minimum number of intervals used in the calculation is one less than the number of events spanned by the age control. However, this assumes that the paleoearthquake events are close in time to the dated deposits used in the computation. Thus where necessary, an extra interval was added to the calculations to account for the possibility that at least one of the events are

Table 11. Recurrence intervals computed for faulting events on the Stagecoach Road fault using composite timing control from trenches SCR-T1 and SCR-T3

[Events are queried where uncertain; ky, thousand years; dashes (--), no data; N/A, not applicable]

\begin{tabular}{|c|c|c|c|c|c|}
\hline \multirow[t]{2}{*}{ Event intervals ${ }^{1}$} & \multirow[t]{2}{*}{$\begin{array}{l}\text { Age-control } \\
\text { alternative }^{2}\end{array}$} & \multirow[t]{2}{*}{$\begin{array}{l}\text { Number of } \\
\text { intervals }^{3}\end{array}$} & \multicolumn{2}{|c|}{$\begin{array}{l}\text { Bounding age constraints } \\
\text { on Intervals }{ }^{4} \\
(k y)\end{array}$} & \multirow{2}{*}{$\begin{array}{c}\begin{array}{c}\text { Estimated } \\
\text { intervals } \\
\text { depositional } \\
\text { rates }^{5} \\
(\mathbf{k y})\end{array} \\
\text { Range }^{8}\end{array}$} \\
\hline & & & Total range ${ }^{6}$ & $\begin{array}{l}\text { Preferred } \\
\text { range }^{7}\end{array}$ & \\
\hline \multicolumn{6}{|l|}{ Individual interval } \\
\hline$Z^{\prime}-Y^{\prime}$ & N/A & 1 & $3-26$ & $10-15$ & $7-14$ \\
\hline$Y^{\prime}-X^{\prime}$ & A & $1-2$ & $0-88$ & $5-30$ & $4-17$ \\
\hline $\mathrm{X}-\mathrm{W}^{\prime}(?)$ & A & $1-2$ & $2-77$ & $15-75$ & $26-67$ \\
\hline$Y^{\prime}-X^{\prime}$ & B & $1-2$ & $0-36$ & $3-15$ & $1-13$ \\
\hline$X^{\prime}-W^{\prime}(?)$ & B & $1-2$ & $0-29$ & $3-15$ & $2-22$ \\
\hline \multicolumn{6}{|l|}{ Average interval } \\
\hline $\mathrm{Z}^{\prime}-\mathrm{X}^{\prime}$ & A & $2-3$ & $3-49$ & $10-20$ & $7-13$ \\
\hline$Z^{\prime}-W^{\prime}(?)$ & A & $3-4$ & $14-37$ & $20-35$ & $20-30$ \\
\hline $\mathrm{Z}^{\prime}-\mathrm{X}^{\prime}$ & B & $2-3$ & $3-24$ & $5-15$ & $5-11$ \\
\hline$Z^{\prime}-W^{\prime}(?)$ & B & $3-4$ & $2-18$ & $5-15$ & $6-13$ \\
\hline \multicolumn{6}{|l|}{ Ash correlation ${ }^{9}$} \\
\hline$Z^{\prime}-Y^{\prime}$ & N/A & $1-2$ & $20-70$ & $20-35$ & -- \\
\hline $\begin{array}{l}\text { between dated deposit } \\
{ }^{6} \text { Total age rang } \\
{ }^{7} \text { Preferred age } r \\
\text { among faulting events } \\
{ }^{8} \text { Interval ages } u \\
\text { computed depositiona } \\
{ }^{9} \text { Recurrence cal }\end{array}$ & $\begin{array}{l}\text { nce intervals of } \\
\text { mative age-cont } \\
\text { 7), whereas Alte } \\
\text { of possible inte } \\
\text { ge constraints fo } \\
\text { urrence interval } \\
\text { ee text). } \\
\text { or intervals using } \\
\text { es for intervals } \\
\text { d dated deposits. } \\
\text { total range of u } \\
\text { tes between date } \\
\text { ation based on as }\end{array}$ & $\begin{array}{l}\text { ents using timir } \\
\text { used in analys } \\
\text { ative } \mathrm{B} \text { is based } \\
\text { als between age } \\
\text { recurrence inter }\end{array}$ & $\begin{array}{l}\text { ontrol from both } \\
\text { f older events. A } \\
\text { the younger TL } \\
\text { is derived from ds }\end{array}$ & $\begin{array}{l}\text { ies listed in tal } \\
\text { ive A uses the } \\
\text { TL-27 and TL } \\
\text { posits in both } \\
\text { on depositior } \\
\text { c and soil-stra } \\
\text { djusted with in }\end{array}$ & $\begin{array}{l}\text { Events listed in } \\
\text { TL and U-series } \\
\text { See text for } \\
\text { hes. } \\
\text { es computed } \\
\text { hic relationships } \\
\text { lations from }\end{array}$ \\
\hline
\end{tabular}


separated in time from the constraining age. For example, Event X' might be considerably younger than the maximum age constraint; the additional interval accounts for the possible extra time. A third alternative where two intervals are added to the minimum number addresses the possibility that both dated deposits are separated from the event horizons by an amount of time equal to the recurrence of individual events. However, this option was not used in the recurrence calculations because the basic assumption that neither dated deposit is close in time to the event horizon did not occur in this study.

These timing uncertainties are also addressed in the computation of paleoearthquake recurrence by using three types of age control (table 11). The first method makes no assumptions and simply uses the upper and lower age bounds for faulting events and event horizons established by the age control. This set of determinations is computed by dividing the time span between dated deposits by the maximum and minimum number of intervals between events.

Additional geologic and soil-stratigraphic constraints are factored into the second, preferred age estimates, and the third set of calculations uses the event ages interpolated from computed depositional rates. These two methods directly use either single or averaged age estimates derived from either geologic-pedogenic constraints or depositional rates.

The recurrence intervals for the five-event scenario depend on which alternative age control is selected. The recurrence intervals between individual events using the preferred Alternative A older age control vary between 0 and $88 \mathrm{ky}$, although the lower bound is geologically unreasonable (table 11). The preferred values range between 3 and $75 \mathrm{ky}$, and commonly between 10 and $30 \mathrm{ky}$. Average recurrences also exhibit a similar wide range of values that vary between 3 and $49 \mathrm{ky}$. Preferred values for average recurrence intervals commonly range between 10 and $30 \mathrm{ky}$. Use of Alternative B with the younger TL age control reduces the individual recurrence intervals to 0 to $36 \mathrm{ky}$, with preferred values on order of 3 to $15 \mathrm{ky}$ (table 11). The values of average recurrence intervals are generally similar. The range of recurrence intervals in table 11 incorporates uncertainties in the ages of bounding units (reported in $\pm 2 \sigma$ error bounds), as well as, where applicable, the number of possible intervening events.

Recurrence intervals do change significantly if ash correlations are used to constrain the timing of events instead of the TL dates (see earlier section on age control of units). Disregarding most the TL ages precludes direct computation of most individual or average recurrence intervals, although the time interval between the two youngest events recorded in the section above the upper ash-bearing unit can be crudely calculated. If a correlation of the ash with the second oldest eruption is accepted, then the time interval between the ash horizon and the youngest event (Event $Z^{\prime}$ ) is $40-70 \mathrm{ky}$. One to two intervals between event $Z^{\prime}$ 'and $Y^{\prime}$ yields recurrence interval estimates of $20-70 \mathrm{ky}$, with preferred values of 20-35 ky (table 11). These intervals serve as a upperbound end member for recurrence intervals, in that they are two to three times longer than recurrence intervals calculated from the TL age control.

The individual recurrence intervals between the last two to three events using the preferred Alternative A age control range from 5 to $30 \mathrm{ky}$ and thus may be shorter by a factors of 30 to 60 percent relative to the oldest event, although the expression and age control of the latter is poorly resolved. However, the estimated longer recurrence intervals in the lower section of the trench are consistent with fewer identifiable events within units spanning a longer interval of time, relative to the upper trench stratigraphy. This pattern, if real, may reflect the threshold of possible weak temporal clustering of fault rupture and associated paleoearthquakes in the latest Pleistocene. However, within the resolution of the timing data, it is equally plausible to postulate that all or part of this pattern may reflect variability in surface faulting that can be accommodated in quasi-periodic or even periodic earthquake recurrence models for intraplate faults with low slip rates (see below). There is no evidence for temporal clustering if the Alternative B set of age control is used. Additional data, particularly regarding the timing patterns of older events, is required to substantiate any possible pattern of temporal clustering.

\section{Fault-Slip Rates}

Fault-slip rates, given in standardized units of millimeters per year, are calculated by dividing the amount of net cumulative apparent displacement of a given reference horizon by the age of the horizon (table 12). The oldest dated unit spanning at least two and preferably three faulting events is selected for the 
reference horizon. Most of the slip rates in table 12 are average values that use time intervals from the present to the ages of displaced horizons with adequate age control. The range in all cited slip rates includes uncertainties in both age control and the net apparent displacements of the reference horizon.

Fault-slip-rate calculations require measurement of the cumulative net apparent displacements of the dated reference horizons. The initial step involves using the displacement per event of table 9 to calculate the cumulative dip-slip displacement of each displaced horizon. Lower bounds for these apparent cumulative displacements are computed by summing the thicknesses of the scarp-derived colluvial wedges, and upper bounds are provided by summation of either the adjusted wedge thicknesses or stratigraphic separations, depending on which is larger. These values are summed for the range of possible fault events interpreted stratigraphically above the reference horizons (for example, two or three events above Unit G5 in trench SCR-T3; tables 8, 12). The amount of cumulative apparent dip-slip displacement also is derived directly by measuring along the fault the amount of dip-slip separation between each dated reference horizon and the stratigraphically highest faulted unit on the footwall.

Net cumulative apparent displacements are derived from the dip-slip values by removing the effects of backtilting and graben formation in the hanging-wall block adjacent to the fault zone (see "Structural Features" section). The effects of secondary deformation are removed by measuring the total observed displacement of each reference horizon along the fault, using projections of each displaced horizon into the fault zone from undeformed or uneroded sections of the hanging-wall and footwall blocks. This correction typically reduces the amount of apparent dip-slip displacements by factors of 0.5 to 0.9 . No correction was applied for oblique slip because only dip-slip slickenlines are observed on the fault zone in the trenches (see "Structural Features" section). Preferred values of net apparent displace-

Table 12. Fault-slip rates for selected dated horizons on Stagecoach Road fault at trenches SCR-T1 and SCR-T3

[Cumulative net tectonic displacements derived from table 9 and composite age control from table 10; ka, thousand years ago; $\mathrm{cm}$, centimeters; $\mathrm{mm} / \mathrm{yr}$, millimeters per year; \pm , plus or minus]

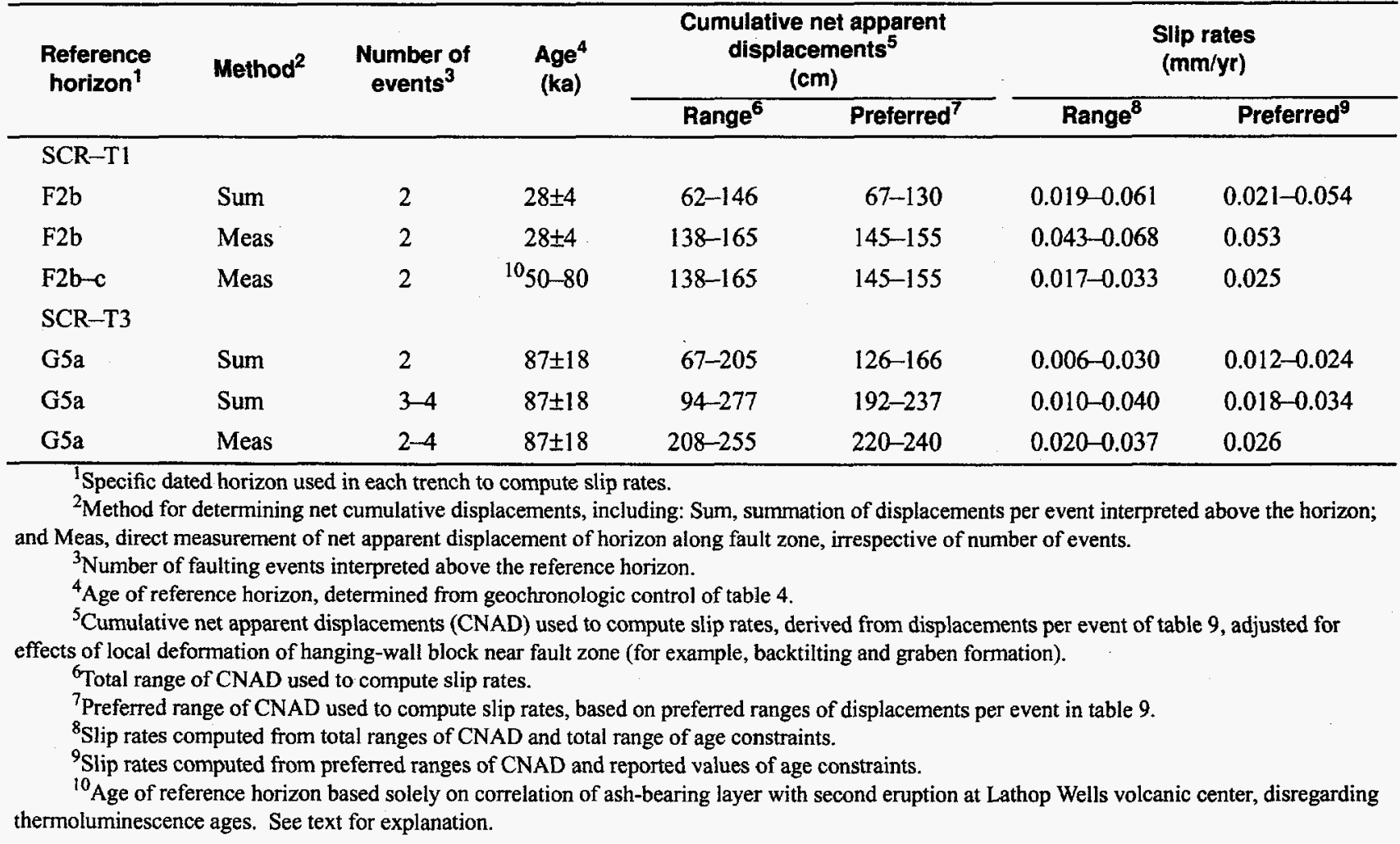


ments are 67 to $166 \mathrm{~cm}$ (two events) and 192 to $240 \mathrm{~cm}$ (three to four events) for a latest Pleistocene and late Pleistocene reference horizons, respectively (table 12).

Fault-slip rates calculated for the latest Pleistocene datum with a TL age of $28 \pm 4 \mathrm{ka}$ varies between 0.02 and $0.07 \mathrm{~mm} / \mathrm{yr}$, with preferred values of 0.02 to $0.05 \mathrm{~mm} / \mathrm{yr}$. Slip rates for an older late Pleistocene horizon with a TL age of $87 \pm 18 \mathrm{ka}$ are two to three times lower, ranging between 0.006 and $0.04 \mathrm{~mm} / \mathrm{yr}$, with preferred values of 0.01 to $0.03 \mathrm{~mm} / \mathrm{yr}$. The increase in slip rates for the youngest time interval reflects in part the possible greater frequency and shorter recurrence intervals for the most recent two to three events noted earlier. Slip rates also were calculated for the latest Pleistocene datum using an age (50-80 ka) derived solely from correlation of the ash-bearing layer in the unit with the second eruption at the Lathrop Wells volcanic center. These rates are low, in the range of 0.017 to 0.033 $\mathrm{mm} / \mathrm{yr}$, with a preferred value of $0.025 \mathrm{~mm} / \mathrm{yr}$. Overall, a slip rate of $0.04 \mathrm{~mm} / \mathrm{yr}$ best characterizes late Pleistocene activity on the Stagecoach Road fault.

\section{Long-Term Displacement Patterns}

Long-term displacement patterns on the Stagecoach Road fault can be inferred from the total offset of volcanic bedrock units across the fault. Additional information can be drawn from the depth to the contact between Miocene volcanic bedrock and overlying poorly-consolidated latest Tertiary to Quaternary sediments, particularly where this contact is associated with a bedrock pediment or erosion surface. The presence of a bedrock pediment between a fault zone and an adjoining bedrock escarpment typically indicates either very low rates of tectonic activity or inactivity on the bounding structure during the formation of the erosion surface.

Interpretations of long-term displacement rates can be extracted directly from subsurface data from the boreholes drilled on the hanging-wall block on the Stagecoach Road fault. The depth to the pumice-rich tuff in borehole SR -3 indicates approximately $99 \mathrm{~m}$ of vertical separation across the Stagecoach Road fault at the SCR-T1 site. This tuff has yielded a $\mathrm{K}-\mathrm{Ar}$ age of $8.5 \pm 3 \mathrm{Ma}$ (Edward McKee, U.S. Geological Survey, written commun, 1994). Long-term average slip rates computed from these data are in the range of 0.009 to $0.02 \mathrm{~mm} / \mathrm{yr}$, with a preferred value of $0.02 \mathrm{~mm} / \mathrm{yr}$.

These rates are considered minimum for several reasons. The unit is exposed only within the bedrock part of the fault zone on the footwall block, although displacements do not appear to be large on the eastern strand of the Stagecoach Road fault at trench SCR-T1. An unknown amount of material may have been eroded from the tuffaceous marker bed on the footwall block during the period of downfaulting of this unit. Deposition of the tuff could precede the initiation of faulting by an unknown amount of time. Also, the presence of an erosion surface across the top of bedrock on the footwall block implies a hiatus in faulting on the Stagecoach Road fault of sufficient length to allow formation of a 0.5 -km-wide pediment.

The absence of significant petrocalcic soils in the hanging-wall sequence within the boreholes near trench SCR-T1 indicates relatively uniform rates of faulting along the Stagecoach Road fault without significant hiatuses within the time interval represented by these deposits. Extrapolation of late Quaternary faulting rates of 0.03 to $0.04 \mathrm{~mm} / \mathrm{yr}$ derived from paleoseismic data in the trenches suggests a speculative, but plausible, age of 3 to $4 \mathrm{Ma}$ for the initiation of downfaulting of the bedrock surface beneath the unconsolidated sediments. In general, this result is consistent with long-term slip rates of 0.02 to $0.03 \mathrm{~mm} / \mathrm{yr}$ on the southern Windy Wash fault at the southwestern corner of Yucca Mountain. These rates are determined from $90 \mathrm{~m}$ of vertical displacement of $3.7 \mathrm{Ma}$ basalts estimated from shallow seismic refraction surveys across the southern Windy Wash fault (Whitney and Berger, U.S. Geological Survey, written commun., 1995).

\section{Rupture Lengths}

No strong evidence exists in the available paleoseismic data for segmentation of the Stagecoach Road fault. Late Pleistocene events correlate well between trenches, particularly in the composite fiveevent scenario with a single old event W' (see discussion in "Age Constraints on Faulting" section). Even the possible absence of events in both trenches in the composite six-event scenario reflects problems in event correlation related to the dating results. Surface ruptures are unlikely to be segmented, given the relatively simple geometry of the fault, the general 
similarity in event timing and amount of displacements per event between the trenches, and the short length of 4 to $8 \mathrm{~km}$ for the mapped surface expression of the fault.

Surface ruptures on the Stagecoach Road fault may continue northward to join with the 4- to 8-kmlong Iron Ridge fault which is a southeastern splay of the Solitario Canyon fault (fig. 2). However, relations exposed in a trench excavated on this strand require that any displacements must be very small $(<5-10 \mathrm{~cm}$ ) and quite young (Holocene to latest Pleistocene)(A.R. Ramelli, Nevada Bureau of Mines and Geology, oral commun., 1995). Timing discrepancies in latest Pleistocene events with the Busted Butte paleoseismic record make it is difficult to continue rupture on the Stagecoach Road fault to the northeast onto the southern Paintbrush Canyon fault (fig. 1; see later section). There is also an absence of fault scarps in the intervening Holocene and late Pleistocene alluvial fans between the two faults (S.C. Lundstrom, U.S. Geological Survey, written commun., 1995). The observed rupture trace on the Stagecoach Road fault likewise does not continue to the southwest of the main zone because of a lack of surface expression in that direction. Surface rupture to the southwest or northeast of the main fault trace, if present, could only consist of minor fracturing or very small displacements that because of erosion or burial do not persist as topographic fault scarps.

A surface rupture length of 4 to $8 \mathrm{~km}$ for the Stagecoach Road fault is anomalously short for the displacements per event based on a comparison with length-displacement relationships of surface ruptures from historical earthquakes (Wells and Coppersmith, 1994). That is, these analyses indicate that displacements per event on the order of 45 to $60 \mathrm{~cm}$ typically are associated with fault lengths of 10 to $20 \mathrm{~km}$. Possible factors that might explain the unusual lengthdisplacement relationships on the Stagecoach Road fault include: (a) the surface displacements are overestimated consistently; (b) the observed surface expression of the fault is a minimum value that is not a good indicator of true rupture length, which is possible if the fault zone continues to the north or south as very small displacements that have been buried or eroded; (c) rupture on the Stagecoach Road fault is associated with faulting on another neighboring fault; (d) fault displacements are anomalously large for rupture lengths, a phenomena observed in some volcanic rift zones (for example, Beanland and others, 1990).
Perhaps large displacements on the Stagecoach Road fault at least in part are related to its proximity to the Lathrop Wells volcanic center.

\section{SUMMARY AND CONCLUSIONS}

The following conclusions regarding the Stagecoach Road fault are based on paleoseismic investigations at trenches SCR-T1 and SCR-T3 (table 13).

Table 13. Summary of paleoseismic parameters for late Quaternary activity on the Stagecoach Road fault

$[<$, less than; ka, thousand years ago; $\mathrm{ky}$, thousand years; $\mathrm{cm}$, centimeters; $\mathrm{mm} / \mathrm{yr}$; millimeters per year]

\begin{tabular}{|c|c|c|c|}
\hline Parameter & Minimum & Maximum & Preferred \\
\hline $\begin{array}{c}\text { Number of } \\
\text { events } \\
(<150- \\
200 \mathrm{ka})\end{array}$ & 3 & 6 & 5 \\
\hline $\begin{array}{c}\text { Displacement } \\
\text { per event }\end{array}$ & $15 \mathrm{~cm}$ & $160 \mathrm{~cm}$ & $\begin{array}{c}30-110 \mathrm{~cm} \\
(\mathrm{Net} 40- \\
70 \mathrm{~cm})\end{array}$ \\
\hline $\begin{array}{l}\text { Age }- \text { most } \\
\text { recent event }\end{array}$ & $5 \mathrm{ka}$ & $15 \mathrm{ka}$ & $11 \mathrm{ka}$ \\
\hline $\begin{array}{l}\text { Recurrence } \\
\text { intervals }\end{array}$ & $5 \mathrm{ky}$ & $75 \mathrm{ky}$ & $10-30 \mathrm{ky}$ \\
\hline $\begin{array}{l}\text { Net cumu- } \\
\text { lative } \\
\text { apparent } \\
\text { displace- } \\
\text { ments }\end{array}$ & $\begin{array}{l}62 \mathrm{~cm} \\
(30 \mathrm{ka}) \\
67 \mathrm{~cm} \\
(90 \mathrm{ka})\end{array}$ & $\begin{array}{l}165 \mathrm{~cm} \\
(30 \mathrm{ka}) \\
240 \mathrm{~cm} \\
(90 \mathrm{ka})\end{array}$ & $\begin{array}{l}140 \mathrm{~cm} \\
(30 \mathrm{ka}) \\
230 \mathrm{~cm} \\
(100 \mathrm{ka})\end{array}$ \\
\hline $\begin{array}{l}\text { Slip rates } \\
\text { (mm/yr) }\end{array}$ & 0.006 & 0.07 & 0.04 \\
\hline
\end{tabular}

1. Both trenches provide evidence for multiple late Quaternary faulting events along a north- to northeast-striking, steeply west-dipping fault zone developed in mixed sequences of late Quaternary colluvium, alluvium, and eolian material.

2. The Stagecoach Road fault is defined in both trenches by a $2-$ to 5 -m-wide zone of carbonatecoated fractures and shears. Secondary deformation, including fracturing, eastward backtilting of strata, and formation of an antithetic graben, is distributed in the hanging wall for 5 to $15 \mathrm{~m}$ west of the main fault zone in both trenches. Additional fracturing and (or) faulting are present in the footwall adjacent to the main fault zone such that the overall width of the Quater- 
nary fault zone and associated deformation approaches 20 to $30 \mathrm{~m}$ (pls. 1-3).

3. The Stagecoach Road fault juxtaposes poorly consolidated sandy deposits of Holocene to latemiddle Pleistocene age ( $\leq 150 \mathrm{ka})$ in the hanging wall against more consolidated middle to early Pleistocene surficial units overlying a bedrock pediment in the footwall. Age estimates are derived from a total of 11 thermoluminescence ages and four U-series disequilibrium ages from units in the hanging-wall blocks of both trenches, combined with soil-stratigraphy in the trenches and correlations to a regional chronosequence developed for the Yucca Mountain area.

4. A composite sequence of at least three, preferably five, and possibly six surface-faulting events is recognized in both trenches on the basis of stratigraphic and structural relationships exposed in the hanging wall adjacent to the fault zone. Criteria used to identify the events include upward termination of fractures, incremental amounts of eastward backtilting of hanging-wall units, and buried scarp-derived colluvial wedges and (or) fissure fills interpreted to be associated with surface rupture and scarp degradation. Two faulting events are identified with a high degree of confidence in the upper part of each trench. Events are more ambiguous and difficult to identify in the lower sections of the trenches. Stratigraphic and structural relationships are generally similar in the hanging walls of both trenches, which facilitates correlation of faulting chronologies at both sites. An additional older set of 1-2 fault ruptures are present along eastern strands of the fault zone within the footwall block of each trench.

5. The amounts of displacements per event in the hanging-wall block are difficult to determine because displaced units do not correlate across the fault zone. Approximate lower- and upperbound estimates of the amount of apparent displacements across the fault zone are derived for each event from the observed thickness of associated colluvial wedges, wedge thicknesses adjusted for effects of footwall degradation, and the total stratigraphic separation between successive event horizons. These bracketing values are augmented by a more restricted range of preferred estimates, which to a large degree rely upon good scientific judgment. Typically, these preferred values are close to the lower range of the stratigraphic separation values. The amount of apparent displacement per event at both sites ranges between extremes of $15 \mathrm{~cm}$ and $160 \mathrm{~cm}$; preferred values are in the range of 30 to $110 \mathrm{~cm}$. Corrections for backtilting and local graben formation reduce the preferred values of net apparent displacements per event to a range of $28-79 \mathrm{~cm}$, and commonly between 45 and $70 \mathrm{~cm}$.

6. A composite fault chronology has been developed from the combined age control in both trenches. The most recent event ( $\left.Z^{\prime}\right)$ is bracketed within a middle Holocene to latest Pleistocene interval (6-15 ka) and probably occurred between 10 and $15 \mathrm{ka}$. A latest Pleistocene age range of $17-32 \mathrm{ka}$ is derived for the penultimate event $\left(Y^{\prime}\right)$, with the most probable occurrence between 22 and $28 \mathrm{ka}$. A possible older event ( $\mathrm{X}^{\prime}$ ) may have occurred between 24 and $105 \mathrm{ka}$, and if so, most likely occurred between 35 and $50 \mathrm{ka}$, using the preferred older of two possible sets of age control. Earlier deformation, indicated by increased backtilting, is difficult to resolve into specific events, although there is evidence for at least one event (W') at this stratigraphic interval in both trenches. The age for this event in the five-event scenario using the older age-control alternative is $69-118 \mathrm{ka}$, with a preferred estimate of 80-110 ka.

An event on the graben-bounding fault in trench SCR-T3 appears to be older (Event V) and must be associated with displacements on the fault zone at an event horizon that extends beneath the bottom of the trench. The ages of the events on the fault zone in the footwall block have not been determined numerically but are considered at least middle to perhaps early Pleistocene in age, based on the well-developed petrocalcic surface soils, which largely postdate deformation.

7. Recurrence intervals are calculated from the combined data on the number of rupture events and age control on faulting, using three different types of age control for events and event horizons. All uncertainties in these data are included in the computations. Using the older of the two age controls, preferred values for individual intervals between the three youngest events with the best age constraints range between 5 and $30 \mathrm{ky}$, whereas recurrence intervals between the next oldest event (W'), 
although poorly constrained, appear longer, ranging from 15 to $75 \mathrm{ky}$. Preferred values of average recurrence intervals including two or more individual inter-event intervals range most commonly between 10 to $30 \mathrm{ky}$.

Recurrence intervals calculated for the two youngest events from correlations of ash-bearing layers in the trenches with the second oldest eruption at Lathrop Wells volcanic center are two to three times longer than those computed from the TL ages alone (that is, 20 to $70 \mathrm{ky}$, with preferred values of 20-35 ky). Recurrence intervals calculated from the older alternative set of TL ages are somewhat shorter for the last two to three events relative to the oldest events in the trenches. This pattern may reflect a tendency for weak temporal clustering, although the variation also may represent periodic or quasi-periodic paleoearthquake recurrence for low-slip, intraplate faults. This pattern is not present if the younger alternative TL ages are used.

8. Fault-slip rates are computed from the amount of cumulative apparent displacements of dated horizons in each trench. These estimates are derived from net cumulative apparent displacements, which are cumulative dip-slip displacements adjusted for the effects of secondary deformation in the hanging wall, including graben formation and eastward backtilting of units. This correction typically reduces the amount of apparent dip-slip displacements by factors of 0.5 to 0.9 , producing values of net cumulative apparent displacements of 62 to $165 \mathrm{~cm}$ and 67 to $277 \mathrm{~cm}$ for latest Pleistocene $(30 \mathrm{ka})$ and late Pleistocene $(90 \mathrm{ka})$ reference horizons, respectively. Fault-slip rates calculated from these data range between 0.006 and $0.07 \mathrm{~mm} / \mathrm{yr}$, with a preferred value of $0.04 \mathrm{~mm} / \mathrm{yr}$.

9. Overall, close correspondence between stratigraphic and faulting relations in both trenches indicates similar fault chronologies in at least the youngest, best-resolved set of faulting events. Therefore, it seems reasonable that surface ruptures on the fault probably extended along all or most of the 4- to 8-km length of the surface trace defined by fault scarps. This inference is consistent with the empirical observation that faults of this length are associated with maximum displacements of 20 to $30 \mathrm{~cm}$ and that larger displacements typical of the Stagecoach
Road fault are related to longer faults (Wells and Coppersmith, 1994). Thus, there is no evidence for fault segmentation along the Stagecoach Road fault; in fact, the surface trace of the fault appears too short for the observed displacements per event. It is possible that at least young surface ruptures may have continued as small fractures or displacements northward to the Iron Ridge fault, a southeastern splay of the Solitario Canyon fault. Alternatively, rupture also may be projected northeastward to join with the southern Paintbrush Canyon fault; however, this scenario would be confined to poorly constrained older events at the Stagecoach Road fault, owing to timing mismatches between the young events at trenches SCR-T1 and SCR-T3, relative to the Busted Butte site on the southern Paintbrush Canyon fault. The Stagecoach Road fault could continue to the southwest toward the Lathrop Wells vent, although there is no fault scarp or other observable manifestation of large surface rupture in this direction.

10. Subsurface data from boreholes drilled in the hanging-wall block to the west of the fault in Trench SCR-T1 indicate a minimum of $99 \mathrm{~m}$ of vertical displacement on the bedrock surface exposed in the footwall of the trench. Minimum long-term slip rates from a displaced volcanic tuff dated at $8.5 \pm 3 \mathrm{Ma}$ are in the range of 0.009 to $0.02 \mathrm{~mm} / \mathrm{yr}$, with a preferred value of $0.02 \mathrm{~mm} / \mathrm{yr}$. The presence of a pediment between the fault and the nearest bedrock ridge to the east suggests a lull or hiatus in tectonic activity between late Miocene deformation and Quaternary faulting. Although speculative, extrapolations of the late Quaternary slip rate to the amount of vertical displacement of the bedrock pediment suggest that the Quaternary faulting may have initiated approximately 3 to $4 \mathrm{Ma}$. The absence of strongly developed petrocalcic soils in the hanging wall deposits found in the boreholes above bedrock imply relatively constant rates of aggradation and faulting on the Stagecoach Road fault throughout this interval of late Pliocene(?)-Quaternary faulting. 


\section{SELECTED REFERENCES}

Allen, C.R., 1986, Seismological and paleoseismological techniques of research in active tectonics, in Active tectonics, studies in geophysics: Washington, D.C., National Academy Press, p. 148-154.

Anderson, L.W., and Klinger, R.E., in press, The Beatty scarp in Nye County, Nevada-An important Late Quaternary morphologic datum: Bulletin of the Seismological Society of America.

Beanland, Sarah, Blick, G.H., and Darby, D.J., 1990, Normal faulting in a back arc basin-Geological and geodetic characteristics of the 1987 Edgecumbe earthquake, New Zealand: Journal of Geophysical Research, v. 95, p. 46934707.

Biasi, G.P., and Weldon, Ray, 1994, Quantitative refinement of calibrated ${ }^{14} \mathrm{C}$ distributions: Quaternary Research, v. 41, p. 1-18.

Birkeland, P.W., 1984, Soils and geomorphology: London, Oxford University Press, $372 \mathrm{p}$.

Bucknam, R.C., and Anderson, R.E., 1979, Estimation of fault scarp age from a scarp-height-slope-angle relationship: Geology, v. 7, p. 11-14.

Carr, M.D., 1990, Styles of extension in the Nevada Test Site region, southern Walker Lane Belt-An integration of volcano-tectonic and detachment fault models, in Wernicke, B.P., ed., Basin and Range extensional tectonics near the latitude of Las Vegas, Nevada: Geological Society of America Memoir 176, p. 283-303.

Coe, J.A., Taylor, E.M., and Schilling, S.P., 1991, Closerange geophotogrammetric mapping of trench walls using multi-model stereo restitution software: American Society of Photogrammetry, Engineering, and Remote Sensing, Technical Papers, v. 5, p. 30-43.

Crone, A.J., Machette, M.N., Bonilla, M.G., Leinkaemper, J.J., Pierce, K.L., and others, 1987, Surface faulting accompanying the Borah Peak earthquake and segmentation of the Lost River fault, central Idaho: Bulletin of the Seismological Society of America, v. 77, no. 3, p. 739-770.

Crowe, Bruce, Morley, Richard, Wells, S.G., Geissman, John, McDonald, Eric, and others, 1992, The Lathrop Wells volcanic center-Status of field and geochronology studies, in International High-Level Radioactive Waste Management Conference, 3d, Las Vegas, Nevada, 1992, Proceedings: La Grange Park, Ill., American Nuclear Society, p. 1997-2013.

Crowe, Bruce, Perry, Frank, Valentine, Greg, Wells, S.G., McFadden, L.D., and others, 1995, Status of volcanism studies for Yucca Mountain Site Characterization project: Los Alamos, N. Mex., Los Alamos National Laboratory Report LAMS-9325, v. 2, 378 p.
dePolo, C.M., and Slemmons, D.B., 1990, Estimation of earthquake size for seismic hazards, in Krinitzky, E.L., and Slemmons, D.B., eds., Neotectonics in earthquake evaluation: Geological Society of America, Reviews in Engineering Geology, v. 8, p. 1-28.

Fairer, G.M., Whitney, J.W., and Coe, J.A., 1989, A closerange photogrammetric technique for mapping neotectonic features in trenches: Bulletin of Association of Engineering Geologists, v. 26, p. 521-530.

Fridrich, C.J., and Price, J.R., 1992, Tectonic framework of Crater Flat basin, adjacent to Yucca Mountain, Nevada - A preliminary report [abs.]: Geological Society of America Abstracts with Proceedings, v. 24, no. 7, p. A189-A190.

Frizzell, V.A., and Shulters, Jacqueline, 1990, Geologic map of the Nevada Test Site, southern Nevada: U.S. Geological Survey Miscellaneous Investigations Series Map I-2046, scale 1:100,000.

Harland, W.B., Armstrong, R.L., Cox, A.V., Craig, L.E., Smith, A.G., and Smith, D.G., 1990, A geologic time scale 1989: New York, Cambridge University Press, $263 \mathrm{p}$.

Hatheway, A.W., and Leighton, F.B., 1979, Trenching as an exploratory method, in Hatheway, A.W., and McClure, C.R., Jr., eds., Geology in the siting of nuclear power plants: Reviews of Engineering Geology, v. 4, p. 169-195.

Hoover, D.L., 1989, Preliminary description of Quaternary and late Pliocene surficial deposits at Yucca Mountain and vicinity, Nye County, Nevada: U.S. Geological Survey Open-File Report 89-359, 45 p.

Ludwig, K.R., 1993, ISOPLOT-A plotting and regression program for radiogenic-isotope data, version 2.70: U.S. Geological Survey Open-File Report 91-445, $42 \mathrm{p}$.

Lundstrom, S.C., Wesling, J.R., Swan, F.H, Taylor, E.M., and Whitney, J.R., 1993, Quaternary allostratigraphy of surficial deposit map units at Yucca Mountain, Nevada-A progress report [abs.]: Geological Society of America Abstracts with Programs, v. 25, no. 5, p. 112.

Lundstrom, S.C., Wesling, J.R., Taylor, E.M., and Paces, J.B., 1994, Preliminary surficial deposits map of the northeast quarter of the Busted Butte 7.5' quadrangle, Nye County, Nevada: U.S. Geological Survey Open-File Report 94-341, scale 1:12,000.

Machette, M.N., 1985, Calcic soils of the southwestern United States, in Weide, D.L., ed., Soils and Quaternary geology of the southwestern United States: Geological Society of America Special Paper 203, p. $1-21$. 
Machette, M.N., 1988, Quaternary movement along the La Jencia fault, central New Mexico: U.S. Geological Survey Professional Paper 1440, $82 \mathrm{p}$.

Machette, M.N., Personius, S.F., and Nelson, A.R., 1992, Paleoseismology of the Wasatch fault zone-A summary of recent investigations, interpretations, and conclusions, in Gori, P.L., and Hays, W.W., eds., Assessment of regional earthquake hazards and risk along the Wasatch Front, Utah: U.S. Geological Survey Professional Paper 1500-A-J, p. A1-A71.

Maldonado, Florian, 1985, Geologic map of the Jackass Flats area, Nye County, Nevada: U.S. Geological Survey Miscellaneous Investigations Series Map I-1519, scale 1:48,000.

McKay, E.J., and Sargent, K.A., 1970, Geologic map of the Lathrop Wells Quadrangle, Nye County, Nevada: U.S. Geological Survey Geologic Quadrangle Map GQ-883, scale $1: 24,000$.

Menges, C.M., Wesling, J.R., Whitney, J.W., Swan, F.H., Coe, J.A., and others, 1994, Preliminary results of paleoseismic investigations of Quaternary faults on eastern Yucca Mountain, Nye County, Nevada, in International High-Level Radioactive Waste Management Conference, 5th, Las Vegas, Nevada, 1994, Proceedings: La Grange Park, Ill., American Nuclear Society, v. 4, p. 2373-2390.

Minor, S.A., Sawyer, D.A., Wahl, R.R., Frizzell, V.A., Jr., Schilling, S.P., and others, 1993, Preliminary geologic map of the Pahute Mesa $30^{\prime} \times 60^{\prime}$ Quadrangle, Nevada: U.S. Geological Survey Open-File Report 93-299, scale 1:100,000.

Muhs, D.R., Whitney, J.W., Shroba, R.R., Taylor, E.M., and Bush, C.A., 1990, Uranium-series dating of secondary carbonates near Yucca Mountain, Nevada—Applications to tectonic, paleoclimatic, and paleohydrologic problems, in International High-Level Radioactive Waste Management Conference, 1st, Las Vegas, Nevada, 1990, Proceedings: La Grange Park, IIl,, American Nuclear Society, p. 924.

Munsell Color Company, Inc., 1990, Munsell soil color charts: Baltimore, $\mathrm{Md}$.

Nelson, Alan, 1992, Lithofacies analysis of colluvial sediments - An aid to interpreting the recent history of Quaternary normal faults in the Basin and Range province, western United States: Journal of Sedimentary Petrology, v. 62, no. 4, p. 607-621.

O'Neill, J.M., Whitney, J.W., and Hudson, M.R., 1992, Photogeologic and kinematic analysis of lineaments at Yucca Mountain, Nevada - Implications for strike-slip faulting and oroclinal bending: U.S. Geological Survey Open-File Report 91-623, 24 p.
Paces, J.B., Menges, C.M., Widmann, Beth, Wesling, J.R., Bush, C.A., and others, 1994, U-series disequilibrium and thermoluminescence ages of paleosols associated with Quaternary faults, east side of Yucca Mountain, in International High-Level Radioactive Waste Management Conference, 5th, Las Vegas, Nevada, 1994, Proceedings: La Grange Park, Ill., American Nuclear Society, v. 4, p. 2391-2401.

Paces, J.B., Taylor, E.M., and Bush, C.A., 1993, Late Quaternary history and uranium isotopic composition of ground water discharge deposits, Crater Flats, Nevada, in International High-Level Radioactive Waste Management Conference, 4th, Las Vegas, Nevada, 1993, Proceedings: La Grange Park, Ill., American Nuclear Society, p. 1573-1580.

Pezzopane, S.K., Menges, C.M., and Whitney, J.R., 1994, Quaternary paleoseismology and Neogene tectonics at Yucca Mountain, Nevada: U.S. Geological Survey Open-File Report 94-568, p. 149-151.

Reiter, Leon, 1990, Earthquake hazard analysis: New York, Columbia University Press, $254 \mathrm{p}$.

Rosholt, J.N., Bush, C.A., Carr, W.J., Hoover, D.L., Swadley, W.C., and Dooley, J.R., 1985, Uranium-trend dating of Quaternary deposits in the Nevada Test Site area, Nevada and California: U.S. Geological Survey Open-File Report 85-540, $72 \mathrm{p}$.

Sawyer, D.A., Fleck, R.J., Lanphere, M.A., Warren, R.G., Broxton, D.E., and Hudson, M.R., 1994, Episodic caldera volcanism in the Miocene southwestern Nevada volcanic field—Revised stratigraphic framework, ${ }^{40} \mathrm{Ar} /{ }^{39} \mathrm{Ar}$ geochronology, and implications for magmatism and extension: Geological Society of America Bulletin, v. 106, no. 10, p. 1304-1318.

Schwartz, D.P., 1988, Geologic characterization of seismic sources-Moving into the 1990's, in von Thun, J.L., ed., Earthquake Engineering and Soil Dynamics; v. 2, Recent Advances in Ground-Motion Evaluation: American Society of Civil Engineers Geotechnical Special Publication 20, p. 1-42.

Schwartz, D.P., and Coppersmith, K.J., 1986, Seismic hazards-New trends in analysis using geologic data, in Active tectonics, studies in geophysics: Washington, D.C., National Academy Press, p. 215-230.

Scott, R.B., 1990, Tectonic setting of Yucca Mountain, southwest Nevada, in Wernicke, B.P., ed., Basin and Range extensional tectonics near the latitude of Las Vegas, Nevada: Geological Society of America Memoir 176, p. 251-282. 
Scott, R.B., 1992, Preliminary geologic map of southern Yucca Mountain, Nye County, Nevada: U.S. Geological Survey Open-File Report 92-266, 28 p., scale 1:12,000.

Scott, R.B., and Bonk, Jerry, 1984, Preliminary geologic map of Yucca Mountain, Nye County, Nevada, with geologic sections: U.S. Geological Survey Open-File Report 84 494, 9 p., scale 1:12,000.

Sieh, Kerry, 1978, Prehistoric large earthquakes produced by slip on the San Andreas fault at Pallett Creek, California: Journal of Geophysical Research, v. 83, no. B8, p. 3907-3939.

Sieh, Kerry, Stuiver, Minze, and Brillinger, David, 1989, A more precise chronology of earthquakes produced by the San Andreas fault in southern California: Journal of Geophysical Research, v. 94, p. 603-623.

Simonds, F.W., and Whitney, J.W., 1993, Quaternary fault distribution at the potential high-level nuclear waste repository at Yucca Mountain, Nevada [abs.]: Geological Society of America Abstracts with Programs, v. 25, no. 6, p. A140-A141.

Simonds, F.W., Whitney, J.W., Fox, K.F., Ramelli, A.R., Yount, J.C., and others, 1995, Fault map of the Yucca Mountain area, Nye County, Nevada: U.S. Geological Survey Miscellaneous Investigations Series Map I-2520, scale 1:24,000.

Soil Survey Staff, 1975, Soil taxonomy-A basic system of soil classification for making and interpreting soil surveys: Washington, D.C., U.S. Government Printing Office, U.S. Department of Agriculture, Soil Conservation Service, Agriculture Handbook 436, 754 p.

Spengler, R.W., and Fox, K.F., Jr., 1989, Stratigraphic and structural framework of Yucca Mountain, Nevada: Radioactive Waste Management and the Nuclear Fuel Cycle, v. 13, p. 21-36.

Swadley, W.C., 1983, Map showing surficial geology of the Lathrop Wells Quadrangle, Nye County, Nevada: U.S. Geological Survey Miscellaneous Investigations Series Map I-1361, scale 1:48,000.

Swadley, W.C., Hoover, D.L., and Rosholt, J.N., 1984, Preliminary report on late Cenozoic faulting and stratigraphy in the vicinity of Yucca Mountain: U.S. Geological Survey Open-File Report 84-788, 42 p.

Swadley, W.C., Yount, J.C., and Harding, S.T., 1988, Reinterpretation of the Beatty Scarp, Nye County, Nevada, in Carr, M.D., and Yount, J.C., eds., Geologic and hydrologic investigations of a potential nuclear waste disposal site at Yucca Mountain, southern Nevada: U.S. Geological Survey Bulletin 1790, p. 113-119.

Swan, F.W., Schwartz, D.P., and Cluff, L.S., 1980, Recurrence of moderate to large magnitude earthquakes produced by surface faulting on the Wasatch fault zone, Utah: Bulletin of the Seismological Society of America, v. 70, p. 1431-146.2.

Szabo, B.J., Carr, W.J., and Gottschall, W.C., 1981, Uranium-thorium dating of Quaternary carbonate accumulations in the Nevada Test Site region, southern Nevada: U.S. Geological Survey Open-File Report $81-119,35 \mathrm{p}$.

Taylor, E.M., 1986, Impact of time and climate on Quaternary soils on the Yucca Mountain area of the Nevada Test Site: Boulder, University of Colorado, Master's thesis, $217 \mathrm{p}$.

Taylor, J.R., 1982, An introduction to error analysis - The study of uncertainties in physical measurements: Mill Valley, Calif., University Science Books, 270 p.

U.S. Department of Energy, 1988, Site characterization plan, Yucca Mountain Site, Nevada Research and Development area, Nevada: U.S. Department of Energy Report DOE/RW-0199, 8 v. [variously paged].

Wallace, R.E., 1977, Profiles and ages of young fault scarps, north-central Nevada: Geological Society of America Bulletin, v. 88, p. 1267-1281.

Wells, D.L., and Coppersmith, K.J., 1994, Updated empirical relationships among magnitude, rupture length, rupture width, rupture area, and surface displacement: Bulletin of Seismological Society of America, v. 84, no. 4, p. 974-1002.

Wesling, J.R., Bullard, T.F., Swan, F.H., Perman, R.C., Angel, M.M., Gibson, J.D., 1992, Preliminary mapping of surficial geology of Midway Valley, Yucca Mountain, Nye County, Nevada: Albuquerque, N. Mex., Sandia National Laboratory Report SAND91-0607, $56 \mathrm{p}$.

Whitney, J.W., Shroba, R.R., Simonds, F.W., and Harding, S.T., 1986, Recurrent Quaternary movement on the Windy Wash fault, Nye County, Nevada [abs.]: Geological Society of America Abstracts with Programs, v. 18 , no. 6, p. 787. 
APPENDIX A 



\section{APPENDIX A. UNIT DESCRIPTIONS FOR SCR-T1}

\begin{tabular}{cccc}
\hline Unit & $\begin{array}{c}\text { Assoc. } \\
\text { Soil }\end{array}$ & Unit Description & $\begin{array}{c}\text { Correla- } \\
\text { tive } \\
\text { strat. unit }\end{array}$ \\
\hline
\end{tabular}

\section{Footwall block}

Tv1 $\quad \mathrm{S}_{\mathrm{B}-\mathrm{A}}$
Silicified tuffaceous sandstone: Pale brown $\left(10 \mathrm{YR} 6 / 3, \mathrm{SiO}_{2}\right)$, white to

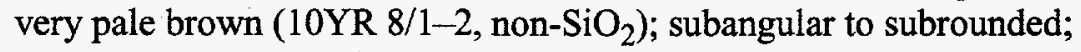 medium to coarse grained; tuffaceous sandstone. Indurated to moderately cemented interbeds with $\mathrm{SiO}_{2}$ and $\mathrm{CO}_{3}$ coatings on fractures. Medium to thickly bedded, with thin to medium interbeds. Base not exposed, but $0.5 \mathrm{~m}$ of tabular shaped unit exposed. Unit dips gently to the west sub-parallel to ground surface.

Tv2 $\quad \mathrm{S}_{\mathrm{B}-\mathrm{A}}$

Pumaceous tephra: White (10YR 8/1); well rounded; well sorted; $15 \%$ lithics-2\% phenocrysts. Stage $\mathrm{I} \mathrm{CO}_{3}$ with weakly cemented interbeds. Thinly interbedded, coarse to very coarse bedding. Base not exposed, but $0.5 \mathrm{~m}$ of tabular shaped unit exposed. In fault contact with unit $\mathrm{F} 1$, unit dips gently west: N15W $10 \mathrm{SW}$.

A1 $\quad$ S $_{\mathrm{B}-\mathrm{A}}$

Mixed colluvium and weathered Tv1: Very pale brown/white (10YR 8/2-1), to pink (7.5YR 7/4 locally); poorly sorted; matrix supported; subangular; gravel. Clasts: $15-30 \mathrm{~cm}$; matrix masked by $\mathrm{CO}_{3}$. Strongly cemented by stage III-IV CO 3 , some $\mathrm{SiO}_{2}$ cementation above contact with Tv1. Poorly bedded; tabular shaped; $0.5 \mathrm{~m}$ thickness. Lower contact is gradational with Tv1. Locally stonelines above weathered bedrock zone. Unit appears to be beveled near scarp on eastern strand of fault zone.

Mixed colluvium and weathered Tv2: Light gray to white (10YR 7/28/1); poorly sorted; matrix supported; subangular to subrounded; pebbly silty sand. Fine pebbles with fine to coarse sand. Weakly to locally moderately cemented with $\mathrm{SiO}_{2}$ and stage $\mathrm{I}-\mathrm{II} \mathrm{CO} 3$. Poorly bedded; irregular tabular shaped; $0-40 \mathrm{~cm}$ thickness. Lower contact is gradational to irregular scoured. Clasts derived primarily from unit Tv2.

B1a B1b $\quad S_{B-A}$

Mixed colluvium and alluvium: White to pink (7.5YR 8/1-7/3); poor to very poorly sorted; variable matrix and clast supported; subangular to subrounded; pebble cobble sandy gravel to sandy pebble gravel.

Clasts: $5-20 \mathrm{~cm}, 10-20 \%$ to $50 \%(\mathrm{Blb})$; matrix masked by $\mathrm{CO}_{3}$. Indurated to moderately cemented stage $\mathrm{IV}-\mathrm{V} \mathrm{CO}_{3}$, with $\mathrm{SiO}_{2}$ and $\mathrm{CO}_{3}$ laminae up to $4 \mathrm{~cm}$ thick. Primary bedding obscured by $\mathrm{CO}_{3}$. Unit $\mathrm{B} 1 \mathrm{~b}$ consists of weakly imbricated channeled gravel lenses, $0.5 \mathrm{~m}$ thick by $4 \mathrm{~m}$ long. Overall unit is tabular; $1.5-3 \mathrm{~m}$ thickness, thickening over fault. Lower contact with units $\mathrm{A} 1 / \mathrm{A} 2$ is gradational to indistinct. Weak tectonic imbrication is present near fault and $\mathrm{CO}_{3}$ cementation increases near main western fault. 


\begin{tabular}{|c|c|c|c|}
\hline Unit & $\begin{array}{l}\text { Assoc. } \\
\text { Soil }\end{array}$ & Unit Description & $\begin{array}{l}\text { Correla- } \\
\text { tive } \\
\text { strat. unit }\end{array}$ \\
\hline B2 & $\mathrm{S}_{\mathrm{B}-\mathrm{A}}$ & $\begin{array}{l}\mathrm{CO}_{3} \text { rubble on K horizon: White }(10 \mathrm{YR} 8 / 1) \text {; poorly sorted; clast } \\
\text { supported; subangular to subrounded; pebble to fine cobble gravel. } \\
\text { Clasts: } 2-15 \mathrm{~cm}, 1-5 \% \text {. Weakly to non cemented by } \mathrm{CO}_{3} \text {. Massive; discon- } \\
\text { tinuous, irregular tabular to lenticular shaped unit } \leq 15 \mathrm{~cm} \text { thick. Sharp } \\
\text { lower contact with unit } \mathrm{B} 1 \mathrm{a} / \mathrm{b} \text {. On the north wall unit thickens above scarp } \\
\text { bevel at main (western) fault zone, and fills fissures and wedges in hanging } \\
\text { wall. }\end{array}$ & $<\mathrm{Q} 1>\mathrm{Q} 5$ \\
\hline $\mathrm{C} 1$ & $\mathrm{~S}_{\mathrm{C}}$ & $\begin{array}{l}\text { Colluvium: Very pale brown to reddish yellow }(10 \mathrm{YR} 7 / 4-7.5 \mathrm{YR} 6 / 6) \text {; } \\
\text { poorly sorted; matrix supported; angular to subrounded; cobble pebble sand. } \\
\text { Clasts: }<15 \mathrm{~cm}, 5-10 \% \text {; matrix: silty fine sand. Strongly to moderately } \\
\text { cemented with } \mathrm{SiO}_{2} \text { and stage } \mathrm{II} \mathrm{CO}_{3} \text {. Massively bedded; irregularly } \\
\text { shaped; } 5-75 \mathrm{~cm} \text { thickness. Sharp to gradational lower contact. Unit infills } \\
\text { pits in } \mathrm{K} \text { horizon. Local krotovinas. }\end{array}$ & $<\mathrm{Q} 1 \geq \mathrm{Q} 4$ \\
\hline $\mathrm{C} 2$ & $\mathrm{~S}_{\mathrm{H}-\mathrm{C}}$ & $\begin{array}{l}\text { Colluvium: Light brown to pink ( } 7.5 \mathrm{YR} 6 / 4-7 / 3) \text {; poorly sorted; matrix } \\
\text { supported; angular to subrounded; pebble sand. Clasts: } 1-6 \mathrm{~cm}, 20 \% \text {; } \\
\text { matrix: silt to medium sand. Weakly to non cemented with } \mathrm{SiO}_{2} . \text { Poorly } \\
\text { bedded; irregularly shaped; } 10-30 \mathrm{~cm} \text { thickness. Gradational to locally } \\
\text { sharp lower contact. Second oldest unit to infill pits in } \mathrm{K} \text { horizon. }\end{array}$ & Q4? \\
\hline
\end{tabular}

\section{Hanging-wall block}

Dla-d $\quad S_{E-D}$

Mixed colluvium and alluvium: Light brown to pink (7.5YR 6/4-7/3); very poorly to poorly sorted; matrix supported; angular to subrounded; pebble sand. Clasts: $1-10 \mathrm{~cm}, 10-20 \%$; matrix: fine to medium sand. Non cemented stage $\mathrm{I} \mathrm{CO}_{3}$. Weakly bedded to massive; tabular shaped; $50 \mathrm{~cm}$ thickness. Internal sub-units have diffuse contacts, lower unit contact is not exposed in trench. Upper subunit thickens to the west from fault. First unit below major rhizolith zone; few thin vertically aligned rhizoliths, project from overlying unit. Unit D16 sampled for TL analysis (TL-27) with age of $49 \pm 1 \mathrm{ka}$.

E1 $\quad S_{\mathrm{E}-\mathrm{D}} \quad$ Mixed colluvium and alluvium: Light brown to pink (7.5YR 6/4-7/3); very poorly to poorly sorted; matrix supported; angular to subrounded; pebble sand. Clasts: $1-10 \mathrm{~cm}, 10-20 \%$; matrix: fine to medium sand. Non cemented stage $\mathrm{ICO}_{3}$. Weakly bedded to massive; tabular shaped; $15-20$ $\mathrm{cm}$ thickness. First unit below major rhizolith zone; few thin vertically aligned rhizoliths, project from overlying unit.

$\mathrm{E} 2 \quad \mathrm{~S}_{\mathrm{E}-\mathrm{D}}$

Scarp-derived colluvium/channel: Light gray (10YR 7/2); poorly sorted; variable matrix and clast supported; angular to subrounded; pebble sand. Clasts: $1-5 \mathrm{~cm}$; matrix: fine to coarse sand. Weakly to moderately cemented by stage $\mathrm{II} \mathrm{CO}_{3}$. Weakly bedded; $1.5 \mathrm{M}$ long lenticular shaped wedge, tapering to the west. $30 \mathrm{~cm}$ maximum thickness. Possible tectonic controlled channel or tectonic wedge, lower contact is concave up and gradational. Tectonic imbrication of clasts is evident along contact with fault. Few, thin rhizoliths are vertically aligned. 


\begin{tabular}{|c|c|c|c|}
\hline Unit & $\begin{array}{l}\text { Assoc. } \\
\text { Soil }\end{array}$ & Unit Description & $\begin{array}{l}\text { Correla- } \\
\text { tive } \\
\text { strat. unit }\end{array}$ \\
\hline$E 3 a-c$ & $\mathrm{~S}_{\mathrm{E}-\mathrm{D}}$ & $\begin{array}{l}\text { Mixed colluvium and alluvium: Very pale brown (10YR 7/3-8/2); poorly } \\
\text { sorted; clast with local matrix supported; angular to subrounded; pebble sand } \\
\text { cobble gravel. Clasts: } 1-10 \mathrm{~cm}, 20-60 \% \text {; matrix: fine to medium sand. } \\
\text { Unit E3a contains sparsely distributed }(5-15 \%) \text { basaltic ash. Weakly to } \\
\text { moderately cemented by stage II- } \mathrm{II}^{+} \mathrm{CO}_{3} \text {. Weak to moderately bedded; } \\
\text { tabular, thinning away from fault; } 20-60 \mathrm{~cm} \text { total thickness. Lower contact } \\
\text { is sharp irregular to more indistinct. Tectonic imbrication of clasts is present } \\
\text { near fault. }\end{array}$ & Q4? \\
\hline E3c & $S_{E-D}$ & $\begin{array}{l}\text { Colluvium/ash interbed: Poorly sorted; matrix supported; angular to } \\
\text { subrounded; pebble sand. Clasts: } 1-4 \mathrm{~cm} \text {; matrix: fine to coarse sand; ash } \\
\text { composition: medium to coarse grained } 5 \% \text { to locally } 20 \% \text {. Weakly to } \\
\text { moderately cemented with stage I-II } \mathrm{CO}_{3} \text {. Forms thin layer near base of } \\
\text { unit E2b; possibly lenticular; } 10 \mathrm{~cm} \text { thickness. Unit discontinuously and } \\
\text { poorly exposed in bench within graben structure. Some rhizoliths. }\end{array}$ & Q4? \\
\hline F1 & $\mathrm{S}_{\mathrm{G}-\mathrm{F}}$ & $\begin{array}{l}\text { Scarp-derived colluvium: Light gray to very pale brown }(10 \mathrm{YR} 7 / 2-3) \text {; } \\
\text { very poorly sorted; predominantly matrix supported; angular to subrounded; } \\
\text { pebble sand to pebble gravel. Clasts: } 1-5 \mathrm{~cm} \text {; matrix: fine to coarse sand. } \\
\text { Weakly to moderately (at base) cemented by stage } \mathrm{I}-\mathrm{I}^{+} \mathrm{CO}_{3} \text {. Massive; } \\
\text { wedge shaped; } 0-25 \mathrm{~cm} \text { thickness. Lower contact is gradational to sharp. } \\
\text { Interpreted as tectonic wedge, with tectonic imbrication of clasts near fault. } \\
\text { Few rhizoliths. }\end{array}$ & Q4-Q5? \\
\hline$F 2 a-c$ & $\mathrm{~S}_{\mathrm{G}-\mathrm{F}}$ & $\begin{array}{l}\text { Mixed colluvium and alluvium: Light yellowish brown to light gray } \\
\text { (10YR 6/4-7/2); poorly sorted; matrix supported; angular to subrounded; } \\
\text { pebble cobble sand. Clasts: } 1-15 \mathrm{~cm}, 10-20 \% \text {; matrix: fine to coarse sand. } \\
\text { Weak to non cemented with stage I-II } \mathrm{CO}_{3} \text {. Weakly bedded; tabular shaped; } \\
70 \mathrm{~cm} \text { maximum total thickness. Lower contact is gradational to sharp. } \\
\text { Contains three sub units, unit F } 2 \mathrm{c} \text { contains } 5-15 \% \text { basaltic ash. Common } \\
\text { rhizoliths and moderately cemented root casts. Unit F } 2 \mathrm{~b} \text { sampled for TL } \\
\text { analysis (TL-16) with age of } 28 \pm 4 \text { ka. }\end{array}$ & Q4-Q5? \\
\hline $\mathrm{F} 2 \mathrm{c}$ & $\mathrm{S}_{\mathrm{G}-\mathrm{F}}$ & $\begin{array}{l}\text { Colluvium/ash interbed: Poorly sorted; matrix supported; angular to } \\
\text { subrounded; pebble sand. Clasts: } 1-5 \mathrm{~cm} \text {; matrix: fine to medium sand; Ash } \\
\text { composition: } \leq 10-15 \% \text {. Weakly cemented with stage } \mathrm{I}^{-} \mathrm{CO}_{3} \text {. Thinly } \\
\text { layered, pinching to the west; } 10-15 \mathrm{~cm} \text { thickness. Lower contact is sharp. }\end{array}$ & Q4-Q5? \\
\hline$F 3 a-b$ & $\mathrm{~S}_{\mathrm{G}-\mathrm{F}}$ & $\begin{array}{l}\text { Mixed colluvium and alluvium: Very pale brown }(10 \mathrm{YR} 7 / 3) \text { to pink } \\
\text { ( } 7.5 \mathrm{YR} 7 / 3) \text {; very poorly sorted; clast supported; angular to subrounded; } \\
\text { sandy cobble pebble gravel. Clasts: } 1-15 \mathrm{~cm}, 10-30 \% \text {; matrix: fine to } \\
\text { coarse sand. Weak to moderately cemented with stage } \mathrm{I}-\mathrm{II} \mathrm{CO}_{3} \text {. Poor to } \\
\text { weakly bedded; tabular shaped; } 50 \mathrm{~cm} \text { thickness. Lower contact is irregular } \\
\text { gradational. Weak tectonic imbrication of clasts is present near fault. Few } \\
\text { rhizoliths. }\end{array}$ & Q4-Q5? \\
\hline
\end{tabular}




\begin{tabular}{|c|c|c|c|}
\hline Unit & $\begin{array}{l}\text { Assoc. } \\
\text { Soil }\end{array}$ & Unit Description & $\begin{array}{l}\text { Correla- } \\
\text { tive } \\
\text { strat. unit }\end{array}$ \\
\hline G1 & $\mathrm{S}_{\mathrm{G}-\mathrm{F}}$ & $\begin{array}{l}\text { Fissure-filling colluvium: Pinkish gray to pink (7.5YR } 7 / 2-3 \text { ); poorly } \\
\text { sorted; matrix supported; angular to subrounded; pebble sand. Clasts: } 1-5 \\
\text { cm; matrix: fine to coarse sand. Weakly cemented with stage } \mathrm{I}-\mathrm{I}^{+} \mathrm{CO}_{3} \text {. Non } \\
\text { bedded; wedge shaped; } 0-15 \mathrm{~cm} \text { thickness. Lower contact is gradational to } \\
\text { indistinct, defined primarily by change in degree of cementation. Interpreted } \\
\text { as tectonic colluvial wedge (unit Gla) and associated fissure fill (unit Glb). } \\
\text { Weak stone line defines top of the unit. }\end{array}$ & Q4-Q5? \\
\hline $\mathrm{G} 2 \mathrm{a}-\mathrm{b}$ & $\mathrm{S}_{\mathrm{G}-\mathrm{F}}$ & $\begin{array}{l}\text { Mixed colluvium and alluvium: Brownish yellow (10YR } 6 / 6) \text { to reddish } \\
\text { yellow ( } 7.5 \mathrm{YR} 6 / 6) \text {; poorly sorted; matrix supported; angular to subrounded; } \\
\text { cobble pebble sand. Clasts: } 1-10 \mathrm{~cm}, 20-50 \% \text {; matrix: fine to coarse sand. } \\
\text { Non cemented, stage } \mathrm{I}^{-} \mathrm{CO}_{3} \text {. Contains two sub-units; tabular shaped; } 20-40 \\
\mathrm{~cm} \text { total thickness. Weak sedimentary imbrication of clasts near fault; lower } \\
\text { contact is sharp and irregular. Unit } \mathrm{G} 2 \mathrm{a} \text { sampled for TL analysis (TL-15) } \\
\text { with anomalous young age of } 3 \pm 0.5 \mathrm{ka} \text {, and thus resampled (TL-25), with } \\
\text { revised age of } 12 \pm 2 \mathrm{ka} \text {. Unit } \mathrm{G} 2 \mathrm{a} \text { is interpreted as scarp-derived colluvial } \\
\text { wedge, with a high percentage of ( } 30-50 \% \text { ) clasts, and thickness of } 0-40 \\
\mathrm{~cm} \text {. Unit } \mathrm{G} 2 \mathrm{a} \text { is displaced by most recent event. }\end{array}$ & Q4-Q5? \\
\hline G3 & $\mathrm{S}_{\mathrm{G}-\mathrm{F}}$ & 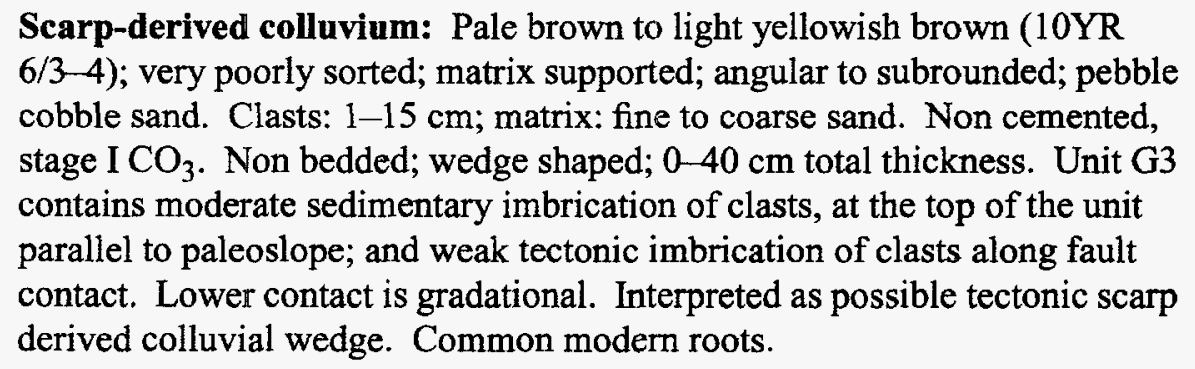 & Q4-Q5? \\
\hline G4 & $S_{G-F}$ & $\begin{array}{l}\text { Mixed colluvium and alluvium: Light yellowish brown (10YR 6/4); very } \\
\text { poorly sorted; matrix supported; angular to subrounded; cobble pebble sand. } \\
\text { Clasts: } 1-12 \mathrm{~cm}, 20-50 \% \text {; matrix: fine to coarse sand with some silt. Non } \\
\text { cemented, with local stage } \mathrm{I}^{-} \mathrm{CO}_{3} \text {. Massive to very poorly bedded; tabular } \\
\text { shaped; } 20-25 \mathrm{~cm} \text { thick unit. Weak to moderate sedimentary imbrication of } \\
\text { clasts throughout unit parallel to ground surface. Lower contact is } \\
\text { gradational. Weak stonelines parallel to ground surface. }\end{array}$ & Q4 Q5? \\
\hline G5 & $\mathrm{S}_{\mathrm{G}-\mathrm{F}}$ & $\begin{array}{l}\text { Mixed colluvium and alluvium: Light yellowish brown (10YR 6/4); } \\
\text { poorly sorted; matrix supported; angular to subrounded; pebble sand. } \\
\text { Clasts: } 1-6 \mathrm{~cm} \text {; matrix: silt to coarse sand. Non cemented, with stage } \mathrm{I}^{--} \\
\mathrm{CO}_{3} \text {. Poorly bedded; lenticular shaped, } 0-20 \mathrm{~cm} \text { thickness. Weak sedimen- } \\
\text { tary, surface parallel imbrication throughout unit. Lower contact is } \\
\text { gradational. Few modern roots. }\end{array}$ & Q4-Q5? \\
\hline
\end{tabular}




\begin{tabular}{|c|c|c|c|}
\hline Unit & $\begin{array}{c}\text { Assoc. } \\
\text { Soil }\end{array}$ & Unit Description & $\begin{array}{c}\text { Correla- } \\
\text { tive } \\
\text { strat. unit }\end{array}$ \\
\hline \multicolumn{4}{|c|}{ Hanging-wall and footwall blocks } \\
\hline $\mathrm{H} 1$ & $\mathrm{~S}_{\mathrm{H}}$ & $\begin{array}{l}\text { Mixed colluvium and alluvium: Pale brown (10YR } 6 / 3) \text {; very poorly } \\
\text { sorted; matrix supported; angular to subrounded; cobble pebble sand. } \\
\text { Clasts: } 1-15 \mathrm{~cm}, 20-30 \% \text {; matrix: fine to coarse sand with some silt. Non } \\
\text { cemented, with stage } \mathrm{I}^{-} \mathrm{CO}_{3} \text {. Poorly bedded; tabular, pinching at footwall; } \\
25-35 \mathrm{~cm} \text { thickness. Weak sedimentary imbrication of clasts near fault. } \\
\text { Lower contact is gradational. Buries most recent event and associated scarp. } \\
\text { Common modern roots. Unit sampled for TL analysis (TL-02 and TL-26) } \\
\text { with ages of } 12 \pm 6 \mathrm{ka} \text { and } 9 \pm 1 \mathrm{ka} \text {, respectively. }\end{array}$ & Q5 \\
\hline $\mathrm{H} 2$ & $\mathrm{~S}_{\mathrm{H}}$ & $\begin{array}{l}\text { Mixed colluvium and alluvium: Pale brown (10YR } 6 / 3) \text {; poor to } \\
\text { moderately sorted; clast with local matrix supported; angular to subrounded; } \\
\text { sandy cobble pebble gravel to gravelly sand. Clasts: } 1-10 \mathrm{~cm}, 15-20 \% \text {; } \\
\text { matrix: fine to coarse sand with some silt. Non cemented, stage I } \mathrm{CO}_{3} \text {. Poor } \\
\text { to moderately bedded; tabular shaped; } 15-30 \mathrm{~cm} \text { thickness. Weak sedimen- } \\
\text { tary imbrication of clasts throughout unit. Bedding of gravels at or above } \\
\text { scarp becomes less well defined away from scarp. Lower contact is sharp to } \\
\text { gradational. Common modern roots. }\end{array}$ & Q5 \\
\hline $\mathrm{H} 3$ & $\mathrm{~S}_{\mathrm{H}} \mathrm{S}_{\mathrm{H}-\mathrm{C}}$ & $\begin{array}{l}\text { Mixed colluvium and alluvium: Pale brown }(10 \mathrm{YR} 6 / 3) \text {; poorly sorted; } \\
\text { matrix supported; angular to subrounded; cobble pebble sand. Clasts: } 1-15 \\
\mathrm{~cm} \text {; matrix: fine to coarse sand with some silt. Non cemented, stage } \mathrm{ICO}_{3} \text {. } \\
\text { Poorly bedded; tabular shaped, pinching at footwall; } \leq 20 \mathrm{~cm} \text { thickness. } \\
\text { Lower contact is gradational. Common modern roots. }\end{array}$ & Q5 \\
\hline H4 & $\mathrm{S}_{\mathrm{H}} \mathrm{S}_{\mathrm{H}-\mathrm{C}}$ & $\begin{array}{l}\text { Eolian sand/colluvium: Pale brown (10YR 6/3); poorly sorted; matrix } \\
\text { supported; angular to subrounded; pebble silty sand (few cobbles) to sandy } \\
\text { silt. Clasts: } 1-10 \mathrm{~cm}, 10-20 \% \text {; matrix: fine to medium sand with silt. Non } \\
\text { cemented. Poorly bedded; tabular shaped; } 20-30 \mathrm{~cm} \text { thickness. Lower } \\
\text { contact is sharp. Platy Av structure with stone line at the surface. }\end{array}$ & Q5-Q6 \\
\hline
\end{tabular}




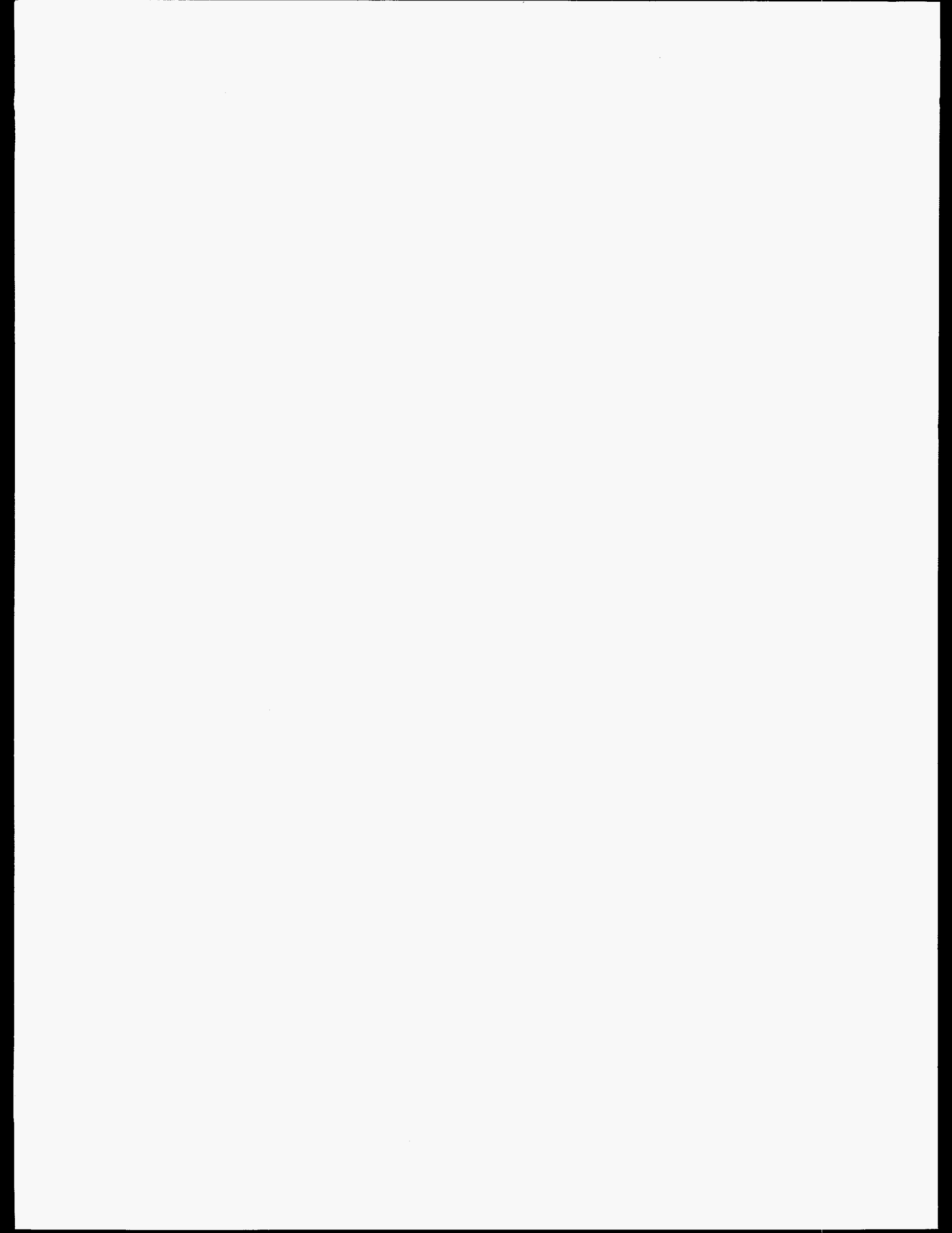


APPENDIX B 



\section{APPENDIX B. UNIT DESCRIPTIONS FOR SCR-T3}

\begin{tabular}{cccc}
\hline Unit & $\begin{array}{c}\text { Assoc. } \\
\text { Soil }\end{array}$ & Unit Description & $\begin{array}{c}\text { Correla- } \\
\text { tive } \\
\text { strat. unit }\end{array}$ \\
\hline
\end{tabular}

\section{Footwall block}

Densely welded tuff: Light brownish gray to grayish brown, 10YR 6-5/2; cryptocrystalline matrix with phenocrysts $1-5 \mathrm{~mm}$. Indurated with $\mathrm{CO}_{3}$ coatings along fractures and exposed surfaces. Massive, tabular shaped. Base not exposed within trench. Displaced by eastern fault (fault A) on west end of unit, and is beveled at the fault.

Mixed colluvium and alluvium: Light brownish gray, 10YR 6/2; poorly sorted; clast supported; angular to subrounded; sandy pebble cobble gravel. Clasts: $1-15 \mathrm{~cm}, 60-70 \%$; matrix: fine to very coarse sand. Weakly cemented with stage II $\mathrm{CO}_{3}$. Weakly bedded, wedge shaped unit. Lower contact is not exposed within the trench, sharp at contact with bedrock. Weak tectonic imbrication of clasts adjacent and parallel to fault.

Moderately developed stone line at top of unit, subparallel and subjacent to bedrock.

Mixed colluvium and alluvium: Pinkish gray, 7.5YR 7/2; very poorly sorted; clast supported; angular to subrounded; silty sandy pebble cobble boulder gravel. Clasts: $1-30 \mathrm{~cm}, 60-70 \%$; matrix: fine to very coarse sand with some silt. Poorly to moderately cemented with stage $\mathrm{II}-\mathrm{II}^{+} \mathrm{CO}_{3}$. Poorly to moderately bedded; irregular shaped unit. Base not exposed within trench, irregular and channeled at east end of trench. Weak tectonic imbrication of clasts along faults at west end of trench. Poorly to moderately developed stonelines defined by coarse cobble boulder deposits.

Mixed colluvium and alluvium: Very pale brown to light gray, 10YR 8/2$7 / 2$; very poorly sorted; clast with local matrix supported; angular to subrounded; sandy pebble cobble gravel. Clasts: $1-20 \mathrm{~cm}, 60-80 \%$; matrix: fine to very coarse sand. Moderately to well cemented by stage II-III $\mathrm{CO}_{3}$. Poorly to moderately bedded; tabular, pinching to the east; $\leq 40 \mathrm{~cm}$ thickness. Channel gravels are 1-2 m thick. Moderate tectonic imbrication of clasts at fault, and weak sedimentary imbrication of clasts in channel deposits.

Lower contact is scoured. Moderately developed cobble stonelines are displaced along fault.

Mixed collevium and alluvium: Very pale brown to light gray, 10YR 8/27/2; very poorly sorted; clast with local matrix supported; angular to subrounded; sandy pebble cobble gravel to local gravelly sand. Clasts: 1-20 $\mathrm{cm}, 50-70 \%$; matrix: fine to very coarse sand. Weak to strongly cemented by stage II-III $\mathrm{CO}_{3}$. Weakly to moderately bedded; tabular pinching to the west; $\leq 80 \mathrm{~cm}$ thickness. Weak sedimentary imbrication of clasts within channel gravels. Lower contact is scoured by some of the stacked channels. Weak to moderately developed stonelines associated with soil. Some rhizoliths project from the overlying unit.

Tiva Cyn Tuff 


\begin{tabular}{|c|c|c|c|}
\hline Unit & $\begin{array}{c}\text { Assoc. } \\
\text { Soil }\end{array}$ & Unit Description & $\begin{array}{l}\text { Correla- } \\
\text { tive } \\
\text { strat. unit }\end{array}$ \\
\hline$B 1_{A-C}$ & $\mathrm{~S}_{\mathrm{D}-\mathrm{B}}$ & $\begin{array}{l}\text { Mixed colluvium and alluvium/scarp-derived colluvium(B1c): Pink to } \\
\text { pinkish gray, } 7.5 \mathrm{YR} 8 / 3-7 / 2 ; \text { poorly sorted; matrix (clast in } \mathrm{B} 1 \mathrm{~b}) \text { supported; } \\
\text { angular to subrounded; cobble pebble sand with some silt. Clasts: } 1-12 \mathrm{~cm} \text {, } \\
10-20 \% \text {; matrix: silt to very coarse sand, mostly fine to medium sand. } \\
\text { Weakly cemented with stage } \mathrm{I}-\mathrm{II}^{-} \mathrm{CO}_{3} \text {. Poorly bedded (moderate in } \mathrm{B} 1 \mathrm{~b} \text { ); } \\
\text { tabular thinning to the east; } 60-110 \mathrm{~cm}(\leq 30 \text { in } \mathrm{B} 1 \mathrm{~b} \text { ) total thickness. Weak } \\
\text { sedimentary imbrication of clasts in unit } \mathrm{B} 1 \mathrm{~b} \text { within channel gravels. Lower } \\
\text { contact is sharp and irregular. Abundant rhizoliths with local root casts as } \\
\text { large as } 10-12 \mathrm{~cm} \text {. Some krotovinas. Unit B la thins across fault and is } \\
\text { beveled across eastern faults (faults A-C) forming a weak scarp. Lower } \\
\text { most unit B1c may contain an indistinct possible scarp derived colluvial } \\
\text { wedge near base. }\end{array}$ & $\geq \mathrm{Q} 3$ \\
\hline $\mathrm{Cl}$ & $S_{D-B}$ & $\begin{array}{l}\text { Mixed colluvium and alluvium: Very pale brown, } 10 \mathrm{YR} 8 / 2 \text {; poorly } \\
\text { sorted; clast supported; angular to subrounded; sandy pebble cobble gravel. } \\
\text { Clasts: } 1-10 \mathrm{~cm}, 60-80 \% \text {; matrix: predominantly fine to medium sand, some } \\
\text { coarse to very coarse sand. Moderately cemented with stage II-III- } \mathrm{CO}_{3} \text {. } \\
\text { Poorly bedded; sigmoidal shaped lens, pinching to the east; } \leq 30 \mathrm{~cm} \\
\text { thickness. Moderately developed sedimentary imbrication of clasts concen- } \\
\text { trated within small channels. Lower contact is sharp. Some scouring into } \\
\text { lower sand unit is evident. Unit preserved only between eastern and western } \\
\text { faults (faults A and D). }\end{array}$ & $\geq \mathrm{Q} 3$ \\
\hline $\mathrm{C} 2$ & $S_{D-B}$ & $\begin{array}{l}\text { Mixed colluvium and alluvium: Very pale brown to light gray, } 10 \mathrm{YR} 8 / 2 \\
7 / 2 \text {; poorly sorted; matrix supported; angular to subrounded; pebble sand. } \\
\text { Clasts: } 1-10 \mathrm{~cm}, 30-40 \% \text {; matrix: fine to very coarse sand. Weakly } \\
\text { cemented with stage } \mathrm{I}-\mathrm{II} \mathrm{CO}_{3} \text {. Poorly bedded, to moderately bedded at taper } \\
\text { above scarp; sigmoidal shaped lens, pinching to the east near fault } \mathrm{A} ; \leq 35 \mathrm{~cm} \\
\text { thickness. Weakly developed tectonic imbrication of clasts along contact } \\
\text { with faults. Lower contact is sharp. Some rhizoliths. }\end{array}$ & $\geq \mathrm{Q} 3$ \\
\hline $\mathrm{C} 3$ & $S_{D-B}$ & $\begin{array}{l}\text { Mixed colluvium and alluvium: Very pale brown, } 10 \mathrm{YR} 8 / 2 \text {; poorly } \\
\text { sorted; clast supported; angular to subrounded; sandy pebble cobble gravel. } \\
\text { Clasts: } 1-10 \mathrm{~cm}, 40-50 \% \text {; matrix: fine to coarse sand. Moderately to } \\
\text { strongly cemented with stage II-III } \mathrm{CO}_{3} \text {. Poorly bedded; lenticular shaped; } \\
\leq 20 \mathrm{~cm} \text { thickness. Possible channel gravel lens within unit. Lower contact } \\
\text { is sharply scoured into underlying unit. Few rhizoliths. }\end{array}$ & $\geq \mathrm{Q} 3$ \\
\hline D1 & $S_{D-B}$ & $\begin{array}{l}\text { Mixed colluvium and alluvium: Very pale brown, } 10 \mathrm{YR} 8 / 3-2 \text {; very } \\
\text { poorly sorted; clast supported; angular to subrounded; sandy pebble cobble } \\
\text { boulder gravel. Clasts: } 1-60 \mathrm{~cm}, 60-80 \% \text {; matrix: fine to very coarse sand. } \\
\text { Moderately to strongly cemented with stage II-IV, predominantly stage III } \\
\mathrm{CO}_{3} \text { Weakest cementation is on the downthrown end of unit across fault. } \\
\text { Poorly bedded to moderately bedded in gravel lenses; tabular shaped, } \\
\text { thickening to the east; } 30-100 \mathrm{~cm} \text { thickness. Moderately developed } \\
\text { sedimentary imbrication of clasts within channel lenses. Lower contact is } \\
\text { sharp and scoured into underlying unit. Few rhizoliths. Few krotovinas at } \\
\text { top of unit. }\end{array}$ & $\geq \mathrm{Q} 3$ \\
\hline
\end{tabular}




\begin{tabular}{|c|c|c|c|}
\hline Unit & $\begin{array}{c}\text { Assoc. } \\
\text { Soll }\end{array}$ & Unit Description & $\begin{array}{l}\text { Correla- } \\
\text { tive } \\
\text { strat. unit }\end{array}$ \\
\hline $\mathrm{D} 2$ & $S_{D-B}$ & $\begin{array}{l}\text { Mixed colluvium and alluvium: Very pale brown to white, } 10 \mathrm{YR} 8 / 2-1 \text {; } \\
\text { poorly sorted; matrix supported; angular to subrounded; pebble sand. Clasts: } \\
1-5 \mathrm{~cm}, 20-30 \% \text {; matrix: fine to medium sand, some coarse sand. Weakly } \\
\text { cemented with stage II } \mathrm{CO}_{3} \text {. Poorly bedded; tabular shaped; } 25-30 \mathrm{~cm} \\
\text { thickness. Weakly developed tectonic imbrication of clasts along contact } \\
\text { with fault. Lower contact is sharp. Unit only preserved in block between } \\
\text { two western faults (faults } \mathrm{C} \text { and D). Some rhizoliths. }\end{array}$ & $\geq \mathrm{Q} 3$ \\
\hline D3 & $S_{D-B}$ & $\begin{array}{l}\text { Mixed colluvium and alluvium: Very pale brown, } 10 \mathrm{YR} 8 / 3-2 \text {; poorly } \\
\text { sorted; clast, with local matrix, supported; angular to subrounded; sandy } \\
\text { pebble cobble gravel. Clasts: } 1-25 \mathrm{~cm}, 40-70 \% \text {; matrix: fine to coarse sand. } \\
\text { Moderately to strongly cemented by stage II-III, predominantly III, } \mathrm{CO}_{3} \text {. } \\
\text { Poorly bedded, base defined by gravel concentration; tabular shaped; } 50-60 \\
\text { cm thickness. Weakly developed sedimentary imbrication of clasts within } \\
\text { gravel subunits. Lower contact is sharp and scoured into lower unit. Unit is } \\
\text { discontinuously preserved above D1 in footwall and between faults C and D. } \\
\text { Local rhizoliths within sandy layers. }\end{array}$ & $\geq \mathrm{Q} 3$ \\
\hline E1 & $\mathrm{S}_{\mathrm{E}}$ & $\begin{array}{l}\text { Colluvium: Very pale brown, } 10 \mathrm{YR} 7 / 3-8 / 2\left(\mathrm{CO}_{3} \text { rich areas }\right) \text {; very poorly } \\
\text { sorted; matrix supported; angular to subrounded; pebble cobble sand. Clasts: } \\
1-25 \mathrm{~cm}, 30-50 \% \text {; matrix: fine to very coarse sand. Strongly cemented with } \\
\text { stage III-IV } \mathrm{CO}_{3} \text {. Poorly bedded to massive; irregularly shaped; highly } \\
\text { variable, } \leq 50 \mathrm{~cm} \text { thickness. Unit fills broad depressions in top of petrocalcic } \\
\text { horizon. Lower contact is sharp. Local krotovinas. }\end{array}$ & $\geq \mathrm{Q} 3$ \\
\hline E2 & $\mathrm{S}_{\mathrm{E}}$ & $\begin{array}{l}\text { Colluvium: Pink to light brown, } 7.5 \mathrm{YR} 7 / 3-6 / 4 \text {; very poorly sorted; matrix, } \\
\text { with local clast, supported; angular to subrounded; pebble cobble sand. } \\
\text { Clasts: } 1-20 \mathrm{~cm}, 20-50 \% \text {; matrix: very fine to very coarse sand with some } \\
\text { silt. Weak to strongly cemented with } \mathrm{SiO}_{2} \text { and stage } \mathrm{II} \mathrm{CO}_{3} \text {. Poorly bedded } \\
\text { to massive; irregularly shaped; highly variable } \leq 65 \mathrm{~cm} \text { thickness. Unit fills } \\
\text { broad depressions in top of petrocalcic horizon. Lower contact is sharp and } \\
\text { irregular. }\end{array}$ & Q4-Q5? \\
\hline
\end{tabular}

Hanging-wall block

F1 $\quad S_{F}$

Mixed colluvium and alluvium: Pinkish gray to pinkish white, 7.5YR 7/2

Q3-Q4?

8/2; poorly sorted; matrix supported; angular to subrounded; pebble cobble sand. Clasts: $1-10 \mathrm{~cm}, 50-70 \%$; matrix: very fine to very coarse sand with some silt. Weak to locally moderately cemented with stage $\mathrm{I}-\mathrm{II}^{-} \mathrm{CO}_{3}$. Tabular shaped unit. Lower contact not exposed within trench. Common rhizoliths.

F2 $\quad \mathrm{S}_{\mathrm{F}}$

Alluvium: Pink to white, 7.5YR 8/3-1; poorly sorted; clast supported; angular to subrounded; cobble pebble sand gravel. Clasts: $1-25 \mathrm{~cm}, 50$ $70 \%$; matrix: fine to coarse sand. Strongly cemented with stage III $\mathrm{CO}_{3}$. Weakly bedded; tabular shaped; $30-40 \mathrm{~cm}$ thickness. Weakly developed sedimentary imbrication of clasts scattered throughout unit. Sharp lower contact. Few rhizoliths. 


\begin{tabular}{|c|c|c|c|}
\hline Unit & $\begin{array}{c}\text { Assoc. } \\
\text { Soil }\end{array}$ & Unit Description & $\begin{array}{l}\text { Correla- } \\
\text { tive } \\
\text { strat. unit }\end{array}$ \\
\hline F3a-b & $\mathrm{S}_{\mathrm{F}}$ & $\begin{array}{l}\text { Alluvium: Lower unit: pink to pinkish white, } 7.5 \mathrm{YR} 8 / 3-2 \text {, upper unit: } \\
7.5 \mathrm{YR} 7 / 2-3 \text {; very poorly sorted; clast supported; angular to subrounded; } \\
\text { sandy cobble pebble gravel. Clasts: } 1-29 \mathrm{~cm}, 50-70 \% \text {; matrix: very fine to } \\
\text { very coarse sand. Weakly to moderately cemented by stage II-III } \mathrm{CO}_{3} \text {. } \\
\text { Weakly to moderately bedded; tabular shaped; } 80-90 \mathrm{~cm} \text { thickness. Weakly } \\
\text { developed sedimentary imbrication of clasts scattered throughout unit. } \\
\text { Lower contact is sharp and scoured into unit below. Weak stonelines } \\
\text { developed at top of the unit. Some rhizoliths. }\end{array}$ & $\mathrm{Q} 3-\mathrm{Q} 4 ?$ \\
\hline F4 & $S_{F}$ & $\begin{array}{l}\text { Alluvium/debris flow?: Pink to pinkish white, } 7.5 \mathrm{YR} 7 / 3-2 \text { to } 8 / 1-2 \text {; very } \\
\text { poorly sorted; Matrix with local clast supported; angular to subrounded; } \\
\text { sandy pebble cobble boulder gravel to gravelly sand. Clasts: } 1-30 \mathrm{~cm} \text {, many } \\
20-30 \mathrm{~cm}, 40-50 \% \text {; matrix: very fine to very coarse sand with some silt. } \\
\text { Weakly to moderately cemented with stage II-III } \mathrm{CO}_{3} \text {. Poorly bedded; } \\
\text { tabular shaped; } 20-30 \mathrm{~cm} \text { thickness. Weakly developed sedimentary } \\
\text { imbrication of clasts scattered throughout unit. Lower contact is sharp to } \\
\text { gradational. Some rhizoliths with local root casts. Possible bevel near } \\
\text { antithetic fault at western graben boundary. }\end{array}$ & Q3-Q4? \\
\hline F5 & $\mathrm{S}_{\mathrm{F}}$ & $\begin{array}{l}\text { Scarp-derived colluvium: Pinkish gray to pinkish white, } 7.5 \mathrm{YR} 7 / 2-8 / 2 \text {, } \\
\text { with local pockets of reddish yellow, } 6 / 6 \text {; very poorly sorted; clast supported; } \\
\text { angular to subrounded; sandy pebble cobble gravel. Clasts: } 1-15 \mathrm{~cm}, 50 \\
60 \% \text {; matrix: very fine to very coarse sand. Weakly to moderately cemented } \\
\text { by stage II-III } \mathrm{CO}_{3} \text {. Poorly bedded; wedge shaped; } \leq 40 \mathrm{~cm} \text { thickness. } \\
\text { Lower contact is gradational and masked by } \mathrm{CO}_{3} \text {. Two subunits comprise } \\
\text { the unit and have stonelines parallel to scarp. Common rhizoliths. May be } \\
\text { scarp derived colluvial wedge for event only exposed in western bounding } \\
\text { fault of graben. }\end{array}$ & Q3-Q4? \\
\hline G1 & $S_{H-G}$ & $\begin{array}{l}\text { Mixed colluvium and alluvium: Pink to pinkish white, } 7.5 \mathrm{YR} 7 / 3-8 / 2 \text {; } \\
\text { very poorly sorted; matrix, with locally clast, supported; angular to } \\
\text { subrounded; sandy pebble cobble gravel to pebble sand (at fault). } \\
\text { Clasts: } 1-20 \mathrm{~cm}, 40-60 \% \text {; matrix: very fine to very coarse sand. Weakly to } \\
\text { moderately cemented with stage II-III } \mathrm{CO}_{3} \text {. Poorly to moderately bedded; } \\
\text { tabular shaped; } 15-35 \mathrm{~cm} \text { thickness, confined to graben. Weakly developed } \\
\text { sedimentary imbrication of clasts within gravel layers. Lower contact is } \\
\text { gradational. Common rhizoliths, with some root casts. Unit buries wedge } \\
\text { F5 to east of western graben bounding fault. Uppermost well-cemented units } \\
\text { within the graben. }\end{array}$ & Q4? \\
\hline $\mathrm{G} 2$ & $\mathbf{S}_{\mathrm{H}-\mathrm{G}} \mathbf{S}_{\mathrm{G}}$ & $\begin{array}{l}\text { Alluvium: Very pale brown, } 10 \mathrm{YR} 7 / 3-8 / 2 \text {; very poorly sorted; matrix } \\
\text { supported; angular to subrounded; pebble cobble sand to sandy gravel. } \\
\text { Clasts: } 1-15 \mathrm{~cm}, 5-60 \% \text {; matrix: very fine to very coarse sand with some } \\
\text { silt. Weakly to moderately cemented with stage I-II } \mathrm{CO}_{3} \text {. Massively } \\
\text { bedded; tabular shaped; } 15-50 \mathrm{~cm} \text { thickness. Lower contact is sharp to } \\
\text { gradational. Common rhizoliths. Unit sampled for TL analysis (TL-29) } \\
\text { with age of } 41 \pm 12 \mathrm{ka} \text {. }\end{array}$ & $\mathrm{Q} 4 ?$ \\
\hline
\end{tabular}




\begin{tabular}{|c|c|c|c|}
\hline Unit & $\begin{array}{l}\text { Assoc. } \\
\text { Soil }\end{array}$ & Unit Description & $\begin{array}{c}\text { Correla- } \\
\text { tive } \\
\text { strat. unit }\end{array}$ \\
\hline G3 & $\mathrm{S}_{\mathrm{H}-\mathrm{G}}$ & $\begin{array}{l}\text { Colluvium, scarp-derived?: Very pale brown, } 10 \mathrm{YR} 7 / 3 \text {; very poorly } \\
\text { sorted; clast to matrix supported; angular to subrounded; sandy pebble } \\
\text { cobble gravel to gravelly sand. Clasts: } 1-20 \mathrm{~cm}, 10-25 \% \text {; matrix: very fine } \\
\text { to very coarse sand. Weakly cemented with stage } \mathrm{I}-\mathrm{II}^{-} \mathrm{CO}_{3} \text {. Weakly } \\
\text { bedded; wedge shaped; } \leq 45 \mathrm{~cm} \text { thickness. Lower contact is poorly exposed } \\
\text { within the trench. Weakly developed tectonic imbrication of clasts along } \\
\text { main fault zone. Interpreted as possible scarp derived colluvial wedge. }\end{array}$ & Q4? \\
\hline G4 & $\mathrm{S}_{\mathrm{H}-\mathrm{G}} \mathrm{S}_{\mathrm{G}}$ & $\begin{array}{l}\text { Mixed colluvium and alluvium: Light yellowish brown to light gray, } \\
\text { 10YR } 6 / 4-7 / 2 \text {; poorly sorted; matrix supported; angular to subrounded; } \\
\text { pebble cobble sand. Clasts: } 1-12 \mathrm{~cm}, 5-20 \% \text {; matrix: very fine to very } \\
\text { coarse sand. Weakly cemented with stage } \mathrm{I}-\mathrm{II}^{-} \mathrm{CO}_{3} \text {. Poorly bedded; tabular } \\
\text { shaped; } 25-50 \mathrm{~cm} \text { thickness. Lower contact is gradational. Common to } \\
\text { many (at west end) rhizoliths. }\end{array}$ & Q4? \\
\hline $\mathrm{G} 5 \mathrm{a}-\mathrm{b}$ & $\mathrm{S}_{\mathrm{H}-\mathrm{G}}$ & $\begin{array}{l}\text { Mixed colluvium and alluvium: Light yellowish brown to very pale } \\
\text { brown, } 10 \text { YR } 6 / 4-7 / 3 \text {; poorly sorted; matrix supported; angular to } \\
\text { subrounded; pebble cobble sand. Clasts: } 1-10 \mathrm{~cm}, 5-10 \% \text {; matrix: very fine } \\
\text { to very coarse sand with some silt. Weakly cemented with stage } \mathrm{ICO}_{3} \text {. } \\
\text { Poorly bedded to massive; tabular shaped; } 40-50 \mathrm{~cm} \text { thickness. Confined to } \\
\text { east end of graben. Lower contact is gradational. Common to few rhizoliths. } \\
\text { Unit sampled for TL dating (TL-19). }\end{array}$ & $\mathrm{Q} 4$ ? \\
\hline $\mathrm{H} 1$ & $\mathrm{~S}_{\mathrm{H}-\mathrm{G}}$ & $\begin{array}{l}\text { Mixed colluvium and alluvium: Light brown to pink to pinkish white, } \\
7.5 \mathrm{YR} 6 / 4-7 / 3 \text { to } 8 / 4-8 / 2 \text {; very poorly sorted; matrix, with locally clast, } \\
\text { supported; angular to subrounded; pebble cobble sand. Clasts: } 1-20 \mathrm{~cm}, 5- \\
15 \% \text {; matrix: very fine to very coarse sand. Weakly to moderately cemented } \\
\text { with stage II- } \mathrm{III} \mathrm{CO}_{3} \text {. Poorly to moderately bedded; tabular shaped; } 15- \\
40 \mathrm{~cm} \text { thickness. Contains gravel lenses approximately } 1 \mathrm{~m} \text { wide by } 10-20 \\
\mathrm{~cm} \text { thick. Weakly developed sedimentary imbrication of clasts within gravel } \\
\text { lenses. Lower contact is gradational and scoured below gravel lenses. Unit } \\
\text { is faulted within graben. Many rhizoliths and root casts. }\end{array}$ & Q4-Q5? \\
\hline $\mathrm{H} 2 \mathrm{a}$ & $\mathrm{S}_{\mathrm{I}-\mathrm{H}}$ & $\begin{array}{l}\text { Scarp-derived colluvium: Pinkish white to white, } 7.5 Y R \quad 8 / 2-1 \text { to } 7 / 3 \text {; } \\
\text { poorly sorted; matrix supported; angular to subrounded; sandy gravel to } \\
\text { pebble cobble sand. Clasts: } 1-15 \mathrm{~cm}, 15-20 \% \text {; matrix: fine to very coarse } \\
\text { sand. Weakly to moderately cemented with stage II-III (local) } \mathrm{CO}_{3} \text {. Poorly } \\
\text { bedded; wedge shaped; } \leq 25 \mathrm{~cm} \text { thickness. Lower contact is gradational. } \\
\text { Sub-unit of } \mathrm{H} 2 \text { interpreted as possible basal facies of scarp derived tectonic } \\
\text { wedge. Many rhizoliths. }\end{array}$ & Q4-Q5? \\
\hline $\mathrm{H} 2 \mathrm{~b}$ & $\mathrm{~S}_{\mathrm{I}-\mathrm{H}}$ & $\begin{array}{l}\text { Scarp-derived colluvium: Pinkish white, } 7.5 \mathrm{YR} 8 / 2 \text { to very pale brown, } \\
10 \mathrm{YR} 7 / 3-8 / 3 \text {; very poorly sorted; matrix supported; angular to subrounded; } \\
\text { pebble cobble sand. Clasts: } 1-25 \mathrm{~cm}, 15-20 \% \text {; matrix: very fine to very } \\
\text { coarse sand. Weakly cemented by stage } \mathrm{I}-\mathrm{II} \mathrm{CO}_{3} \text {. Poorly bedded; wedge } \\
\text { shaped; } \leq 20 \mathrm{~cm} \text { thickness. Moderately developed sedimentary imbrication } \\
\text { of clasts near fault. Lower contact is gradational, and poorly exposed due to } \\
\text { bench. Sub-unit of } \mathrm{H} 2 \text { interpreted as possible upper facies of scarp derived } \\
\text { tectonic wedge. }\end{array}$ & Q4-Q5? \\
\hline
\end{tabular}




\begin{tabular}{|c|c|c|c|}
\hline Unit & $\begin{array}{c}\text { Assoc. } \\
\text { Soil }\end{array}$ & Unit Description & $\begin{array}{l}\text { Correla- } \\
\text { tive } \\
\text { strat. unit }\end{array}$ \\
\hline $\mathrm{H} 3 \mathrm{a}-\mathrm{b}$ & $\mathrm{S}_{\mathrm{I}-\mathrm{H}}$ & $\begin{array}{l}\text { Colluvium: Pink, } 7.5 \text { YR } 7 / 3 \text {; poorly sorted; matrix supported; angular to } \\
\text { subrounded; pebble cobble sand. Clasts: } 1-12 \mathrm{~cm} \text {, few cobbles, } 10-15 \% \text {; } \\
\text { matrix: very fine to very coarse sand, predominantly fine to medium sand; } \\
\text { contains sparse ash in basal sub-unit: very fine to fine, highly dispersed }<5 \% \text {. } \\
\text { Weakly cemented with stage } \mathrm{III}^{-} \text {(locally) } \mathrm{CO}_{3} \text {. Poorly bedded to massive; } \\
\text { tabular or wedge shaped; } \leq 20 \mathrm{~cm} \text { thickness. Unit poorly exposed in bench. } \\
\text { Lower contact is gradational? (poorly exposed). Few rhizoliths. }\end{array}$ & Q4-Q5? \\
\hline $\mathrm{H} 3 \mathrm{c}$ & $\mathrm{S}_{\mathrm{I}-\mathrm{H}}$ & $\begin{array}{l}\text { Mixed colluvium and alluvium: Light brown to pinkish white, } 7.5 \mathrm{YR} \\
6 / 3-8 / 2 \text {; very poorly sorted; matrix supported; angular to subrounded; } \\
\text { pebble cobble sand. Clasts: } 1-10 \mathrm{~cm} \text {, few cobbles, } 10-15 \% \text {; matrix: very } \\
\text { fine to very coarse sand, predominantly fine to medium sand, with some silt. } \\
\text { Weakly to moderately cemented with stage } \mathrm{I}-\mathrm{II}^{+} \mathrm{CO}_{3} \text {. Poorly bedded; } \\
\text { tabular shaped; } 30-50 \mathrm{~cm} \text { thickness. Lower contact is gradational. Last unit } \\
\text { to be deformed within graben. Common rhizoliths in } \mathrm{CO}_{3} \text { cemented areas. }\end{array}$ & $\mathrm{Q} 4$ \\
\hline
\end{tabular}

\section{Hanging-wall and Footwall blocks}

Ila-b $\quad \mathrm{S}_{\mathrm{l}-\mathrm{H}}$

Scarp-derived colluvium: Light brown, 7.5YR 6/3; very poorly sorted; matrix supported; angular to subrounded; boulder cobble pebble sand. Clasts: $1-35 \mathrm{~cm}$, few boulders, $10-30 \%$; matrix: very fine to very coarse sand with some silt. Weakly cemented with stage $\mathrm{ICO}_{3}$. Weakly to moderately bedded; wedge shaped; 0-60 cm thickness. Moderately developed sedimentary imbrication of clasts inclined away from fault. Lower contact is gradational. Few very weak rhizoliths.

Mixed colluvium and alluvium: Light brown to pinkish gray, $7.5 \mathrm{YR}$ 6/4-7/2; poorly sorted; matrix supported; angular to subrounded; pebble cobble sand with minor silt. Clasts $1-20 \mathrm{~cm}, 5-25 \%$; matrix: very fine to very coarse, predominantly fine to medium sand with some silt. Weakly cemented with stage $\mathrm{I}-\mathrm{II}^{-} \mathrm{CO}_{3}$. Poorly bedded; tabular shaped; $15-60 \mathrm{~cm}$ thickness. Unit thickens within graben. Lower contact is gradational. Weak to moderately developed stonelines at top of unit. Few rhizoliths near fault. Oldest unit not deformed by graben forming faults.

Mixed colluvium and alluvium: Light brown to reddish yellow, 7.5YR 6/4-6/6 $\left(\mathrm{SiO}_{2}\right.$ nodules); very poorly sorted; matrix supported; angular to subrounded; pebble cobble sand. Clasts: $1-25 \mathrm{~cm}, 20-25 \%$; matrix: very fine to very coarse sand, predominantly fine to medium sand. Weakly to moderately bedded; tabular shaped, pinching out to the west of graben; $\leq 50 \mathrm{~cm}$ thickness. Weakly developed sedimentary imbrication of clasts scattered throughout unit. Lower contact is scoured and sharp to locally gradational. Few rhizoliths. Some $\mathrm{SiO}_{2}$ nodules at west end of unit. Base sampled for TL dating (TL-18) with age of $22 \pm 5 \mathrm{ka}$. 


\begin{tabular}{|c|c|c|c|}
\hline Unit & $\begin{array}{l}\text { Assoc. } \\
\text { Soil }\end{array}$ & Unit Description & $\begin{array}{l}\text { Correla- } \\
\text { tive } \\
\text { strat. unit }\end{array}$ \\
\hline $\mathrm{I} 4 \mathrm{a}-\mathrm{b}$ & $\overline{\mathrm{S}_{\mathrm{J}-\mathrm{I}}}$ & $\begin{array}{l}\text { Fissure-fill and scarp-derived colluvium: Light yellowish brown to very } \\
\text { pale brown, 10YR } 6 / 4-7 / 3 \text {; very poorly sorted; clast, with local matrix, } \\
\text { supported; angular to subrounded; sandy cobble pebble boulder gravel. } \\
\text { Clasts: } 1-30 \mathrm{~cm}, 30-40 \% \text {; matrix: very fine to very coarse sand. Weakly } \\
\text { bedded; lenticular/wedge shaped; } \leq 45 \mathrm{~cm} \text { thickness. Moderately developed } \\
\text { sedimentary imbrication of clasts throughout unit; strongly developed } \\
\text { tectonic imbrication of clasts along contact with fault. Lower contact is } \\
\text { sharp to gradational and scoured. Few rhizoliths in fissure. Unit I4b may } \\
\text { combine with units } \mathrm{J} 2 \text { and J3 as basal detritus facies of composite wedge } \\
\text { deposited above fissure fill (unit I4a) in preferred interpretation. Alterna- } \\
\text { tively, unit I4a may be interpreted as channel influenced by formation of } \\
\text { scarp. }\end{array}$ & Q4-Q5? \\
\hline $\mathrm{J} 1$ & $\mathrm{~S}_{\mathrm{J}_{-I}}$ & $\begin{array}{l}\text { Mixed colluvium and alluvium: Reddish yellow to pink, } 7.5 \mathrm{YR} 6 / 6-7 / 4 \text {; } \\
\text { very poorly sorted; angular to subrounded; pebble cobble sand to silty sand } \\
\text { (away from fault). Clasts: } 1-22 \mathrm{~cm}, 5-40 \% \text {; matrix: very fine to very coarse } \\
\text { sand, predominantly fine to medium, with some silt. Very weakly cemented } \\
\text { with stage } \mathrm{I} \mathrm{CO}_{3} \text {. Poorly bedded; tabular shaped; } 15-60 \mathrm{~cm} \text { thickness. } \\
\text { Weakly developed sedimentary imbrication of clasts scattered throughout } \\
\text { unit. Lower contact is gradational. Unit includes buried Av soil horizon. } \\
\text { Unit I5 appears to be channeled into unit I4 at the fault. }\end{array}$ & Q4-Q5? \\
\hline $\mathbf{J} 2$ & $\mathrm{~S}_{\mathrm{J}-\mathrm{I}}$ & $\begin{array}{l}\text { Scarp-derived colluvium: Very pale brown, } 10 \mathrm{YR} 7 / 4 \text {; very poorly sorted; } \\
\text { matrix supported; angular to subrounded; pebble cobble sand to silty sand. } \\
\text { Clasts: } 1-22 \mathrm{~cm}, 15-25 \% \text {; matrix: very fine to very coarse sand, predomi- } \\
\text { nantly fine to medium sand, with silt. Very weakly cemented with stage I } \\
\mathrm{CO}_{3} \text {. Poorly bedded; wedge shaped; } \leq 40 \mathrm{~cm} \text { thickness. Weakly developed } \\
\text { sedimentary imbrication of clasts near fault. Lower contact is sharp. }\end{array}$ & Q5 \\
\hline $\mathrm{J3}$ & $\mathrm{S}_{\mathrm{J}-\mathrm{I}}$ & $\begin{array}{l}\text { Scarp-derived colluvium: Very pale brown, } 10 \mathrm{YR} 7 / 4-7 / 3 \text {; moderately } \\
\text { sorted; matrix supported; angular to subrounded; pebble silty sand. Clasts: } \\
1-6 \mathrm{~cm}, 5-10 \% \text {; matrix: very fine to very coarse sand, predominantly very } \\
\text { fine to fine sand. Non cemented, stage } \mathrm{I}^{-} \mathrm{CO}_{3} \text {. Massively bedded; wedge } \\
\text { shaped; } \leq 15 \mathrm{~cm} \text { thickness. Lower contact is gradational. Interpreted as } \\
\text { upper part of youngest scarp-derived wedge from most recent event. }\end{array}$ & Q5 \\
\hline J4 & $\mathrm{S}_{\mathrm{J}-\mathrm{I}}$ & $\begin{array}{l}\text { Mixed colluvium, alluvium, and eolian sand: Light yellowish brown, } \\
10 \text { YR } 6 / 4 \text {; poorly sorted; matrix supported; angular to subrounded; pebble } \\
\text { cobble silty sand. Clasts: } 1-26 \mathrm{~cm}, 5-20 \% \text {; matrix: very fine to very coarse } \\
\text { sand, predominantly fine to very fine sand. Non cemented, stage } \mathrm{I}^{-} \mathrm{CO}_{3} \text {. } \\
\text { Poorly to moderately bedded; tabular shaped; } 10-35 \mathrm{~cm} \text { thickness. Weakly } \\
\text { developed sedimentary imbrication of clasts within channels. Lower contact } \\
\text { is sharp to gradational. } \mathrm{J} 1 \text { is the only unfaulted unit in trench. Unit does } \\
\text { contain fracture with no displacement above fault. Unit sampled for TL } \\
\text { dating, west of the fault (TL-17), with erroneously old age of } 35 \pm 5 \text {; thus unit } \\
\text { resampled (TL-28), with revised age of } 2 \pm 0.4 \mathrm{ka} \text {. }\end{array}$ & $\leq \mathrm{Q} 5$ \\
\hline
\end{tabular}


Page(s) size did not permit electronic reproduction. Information may be purchased by the general public from the National Technical Information Service, U.S. Department of Commerce, Springfield, VA 22161 (Area Code 703-487-4650). DOE and DOE contractors may purchase information by contacting DOE's Office of Scientific and Technical Information, P.O. Box 62, Oak Ridge, TN 37831, Attn: Information Services (Area Code 423-576-8401). 